\title{
A Comparative NMR Study of the Polypeptide Backbone Dynamics of Hemoglobin in the Deoxy and Carbonmonoxy Forms
}

$$
\text { by }
$$

Xiang-jin Song, Yue Yuan, Virgil Simplaceanu, Sarata Chandra Sahu, Nancy T. Ho, and Chien Ho*

Department of Biological Sciences, Carnegie Mellon University, 4400 Fifth Avenue, Pittsburgh, PA 15213

\section{SUPPORTING INFORMATION}

Figure 1S. Order parameters $\left(\mathrm{S}^{2}\right)$ of amide ${ }^{15} \mathrm{~N}-{ }^{1} \mathrm{H}$ bonds in $\mathrm{Hb}$ at $34{ }^{\circ} \mathrm{C}$.

Figure $2 \mathrm{~S}$. $\mathrm{R}_{\mathrm{ex}}$ terms derived from Model-free analysis for deoxy-Hb A and $\mathrm{HbCO}$ A at $29^{\circ} \mathrm{C}$.

Figure 3S. X-ray crystallographic B-factors (filled circles) and NMR order parameters (open circles, obtained at $29^{\circ} \mathrm{C}$ ). Top: deoxy-Hb A, with B-factors from 1A3N (PDB 
ID); Bottom: $\mathrm{HbCO} A$, with B-factors from 1BBB. The numbering of the amino acid residues of $\beta$-chain starts from No.152.

Figure 4S. NMR relaxation parameters of backbone amide ${ }^{15} \mathrm{~N}$ in $\mathrm{Hb}$ A at $29{ }^{\circ} \mathrm{C}$ (filled circle) and $34{ }^{\circ} \mathrm{C}$ (open circle), under following conditions: (1) deoxy Hb A at $11.7 \mathrm{~T}$; (2) deoxy $\mathrm{Hb} \mathrm{A}$ at $14.1 \mathrm{~T}$; (3) HbCO A at 11.7 T; (4) HbCO A at 14.1 T.

Figure $5 S . R_{1}$ and $R_{2}$ relaxation decay curves for amide ${ }^{15} \mathrm{~N}$ in two amino acid residues ( $\alpha 31 \mathrm{Arg}$ and $\beta 146 \mathrm{His}$ ) at $29^{\circ} \mathrm{C}$. Repeated data points are indicated by arrows.

Table 1S. NMR Relaxation Parameters of Backbone Amide ${ }^{15} \mathrm{~N}$ in Deoxy-Hb A

Table 2S. NMR Relaxation Parameters of Backbone Amide ${ }^{15} \mathrm{~N}$ in $\mathrm{HbCO} \mathrm{A}$

Table 3S. Amino Acid Residues in $\mathrm{Hb}$ A Selected for Correlation Time Determination

This material is available free of charge via the Internet at http://pubs.acs.org 
Figure 1S
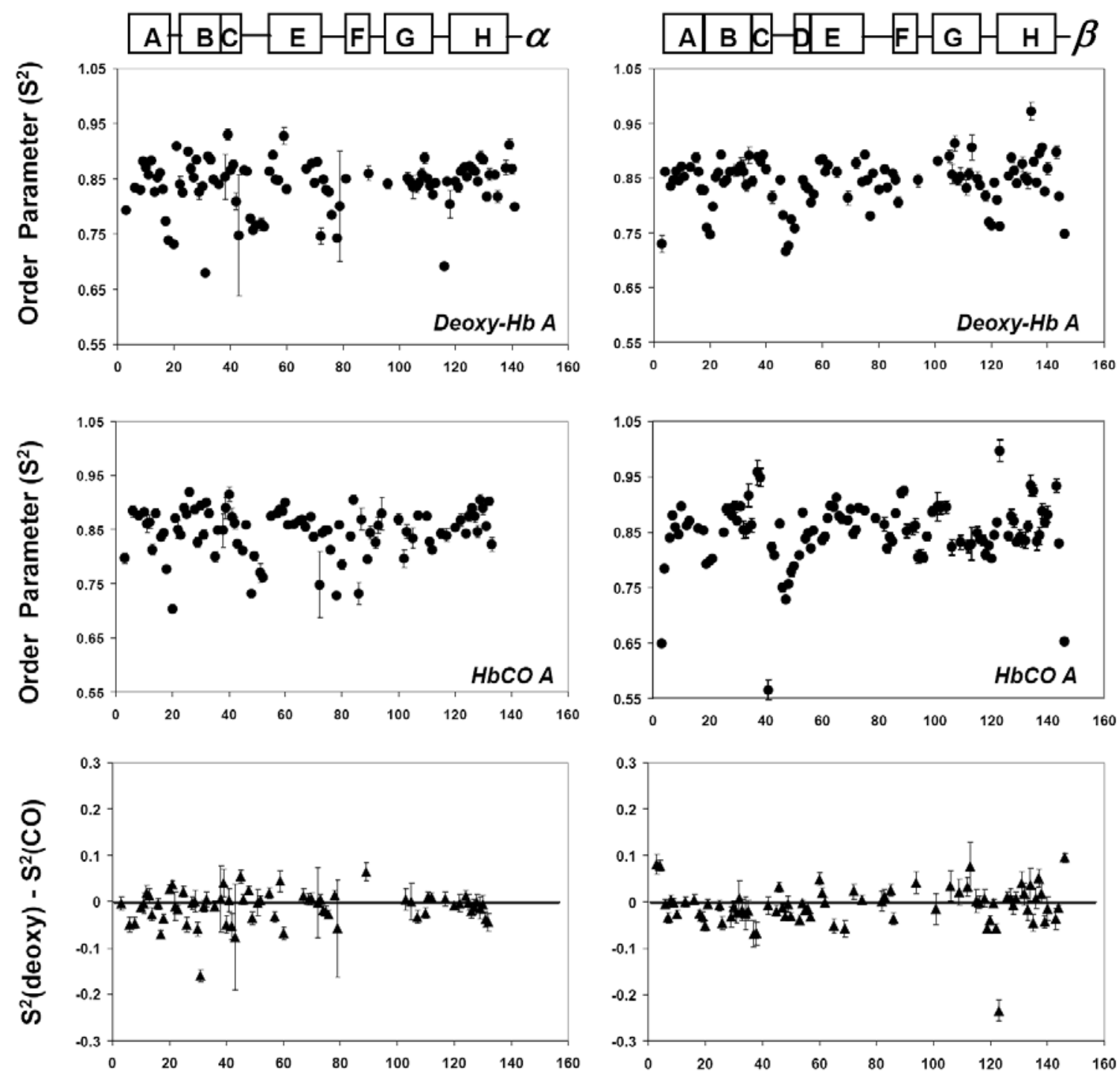

Amino Acid Residue Number

Amino Acid Residue Number 
Figure 2S
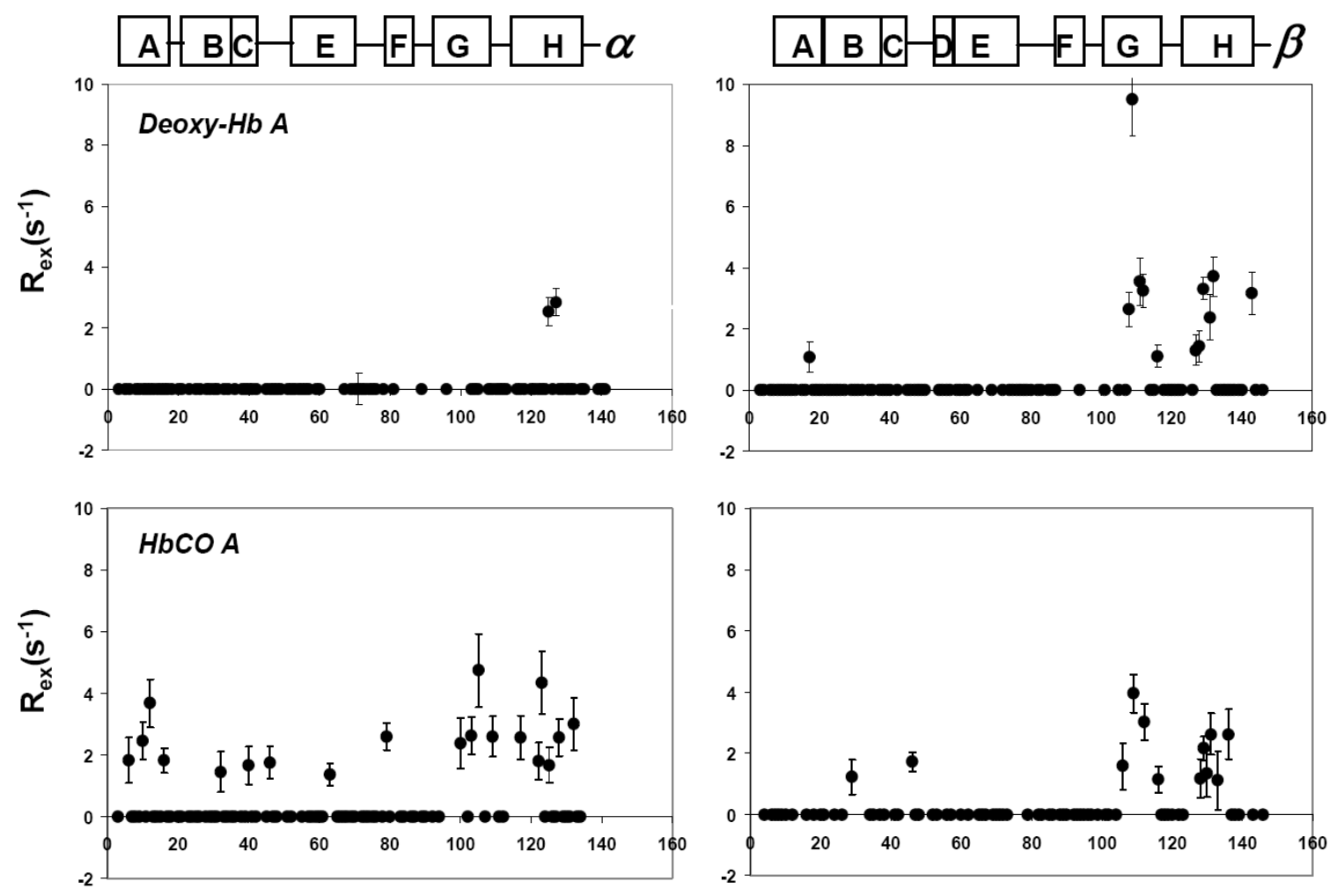

Amino Acid Residue Number

Amino Acid Residue Number 
Figure 3S
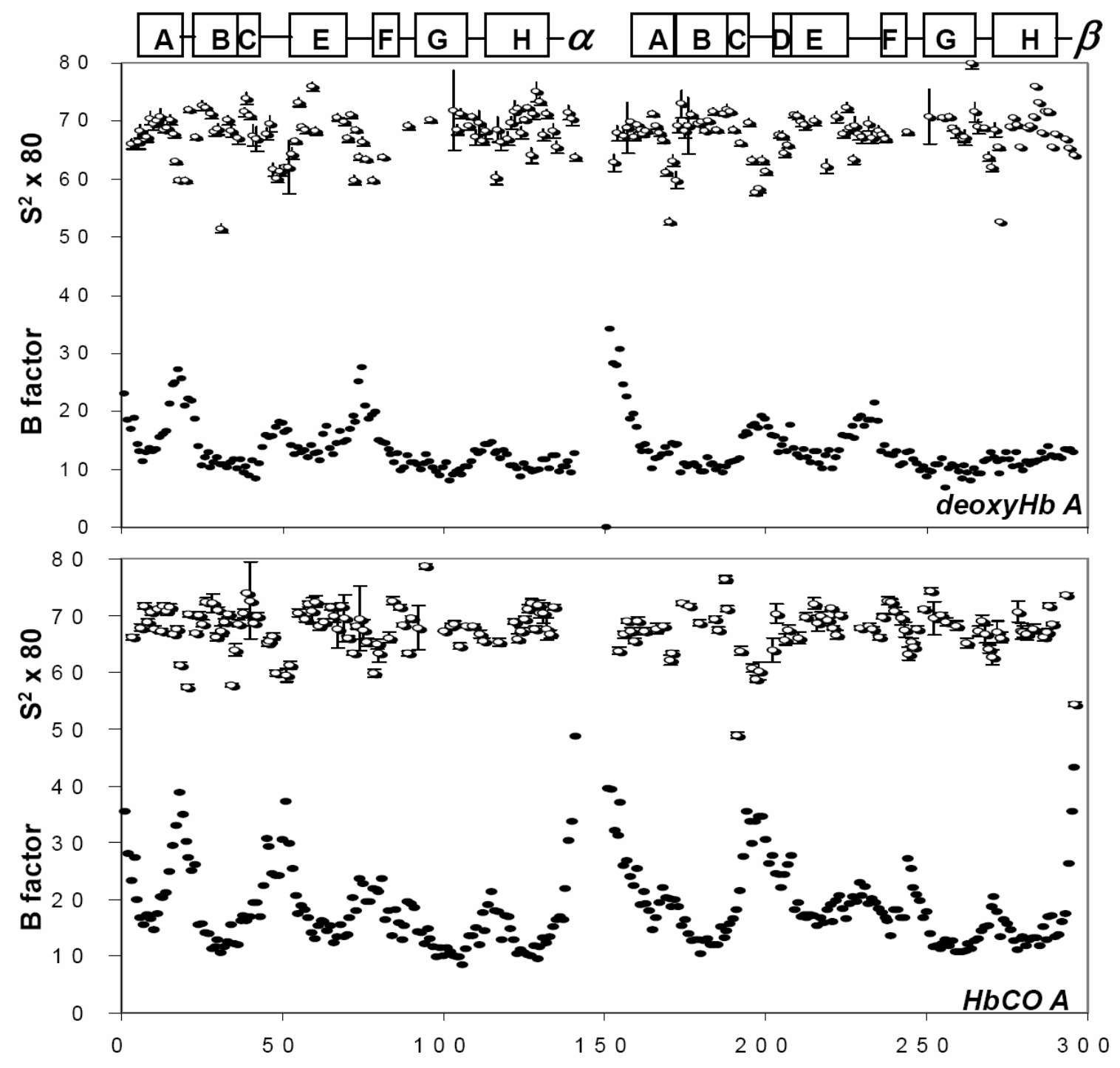

Amino Acid Residue Number 
Figure 4S (1)
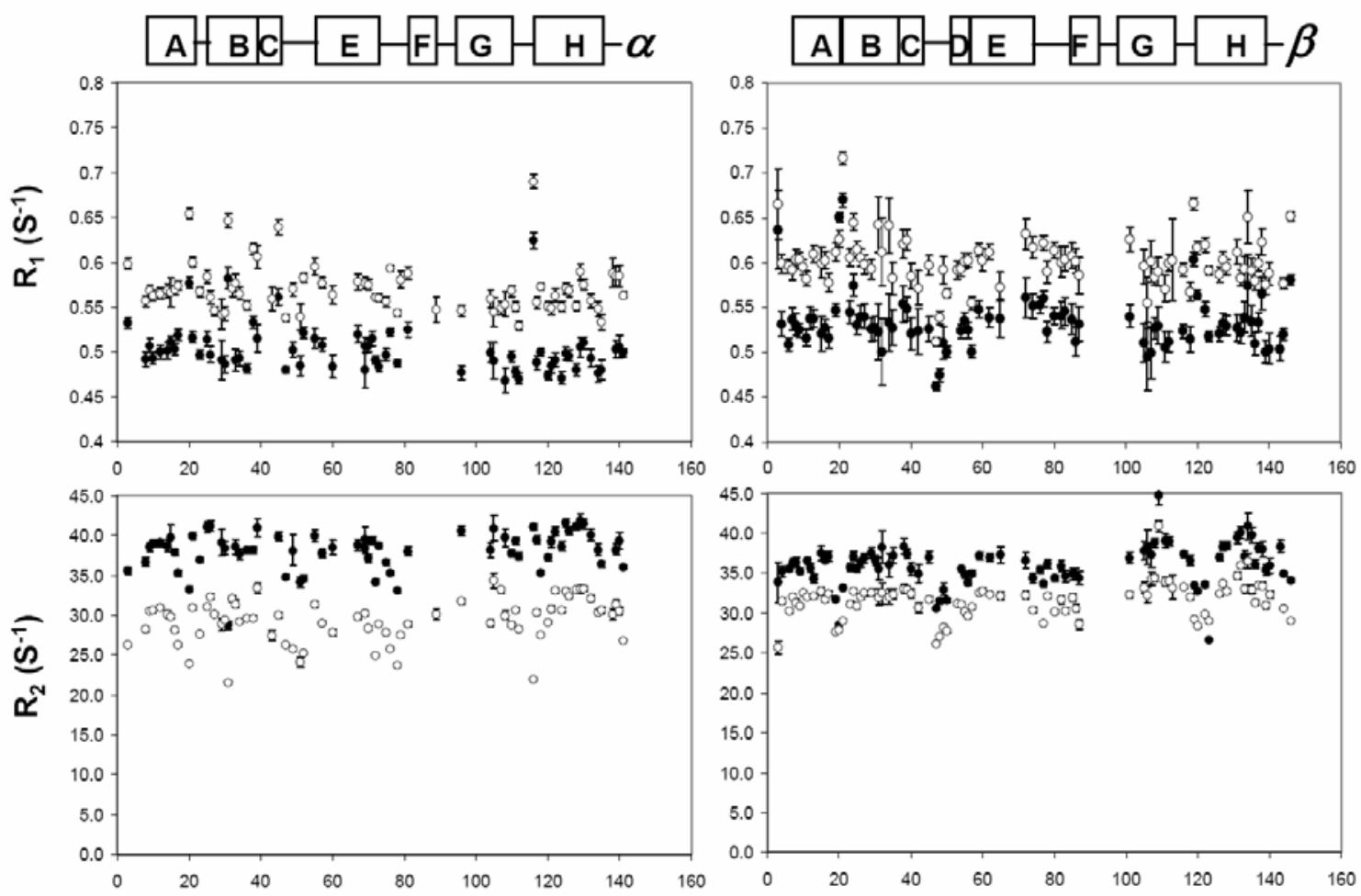

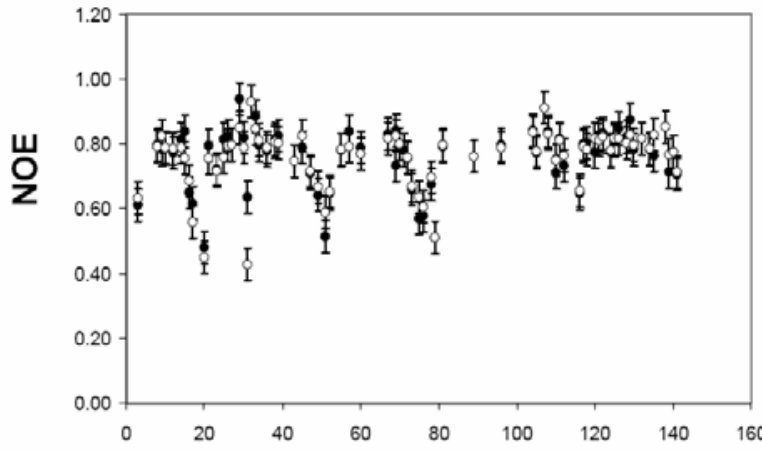

Amino Acid Residue Number

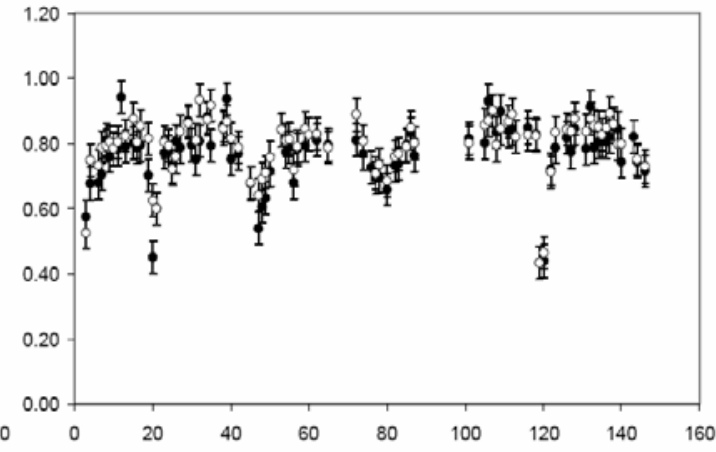

Amino Acid Residue Number 
Figure 4S (2)
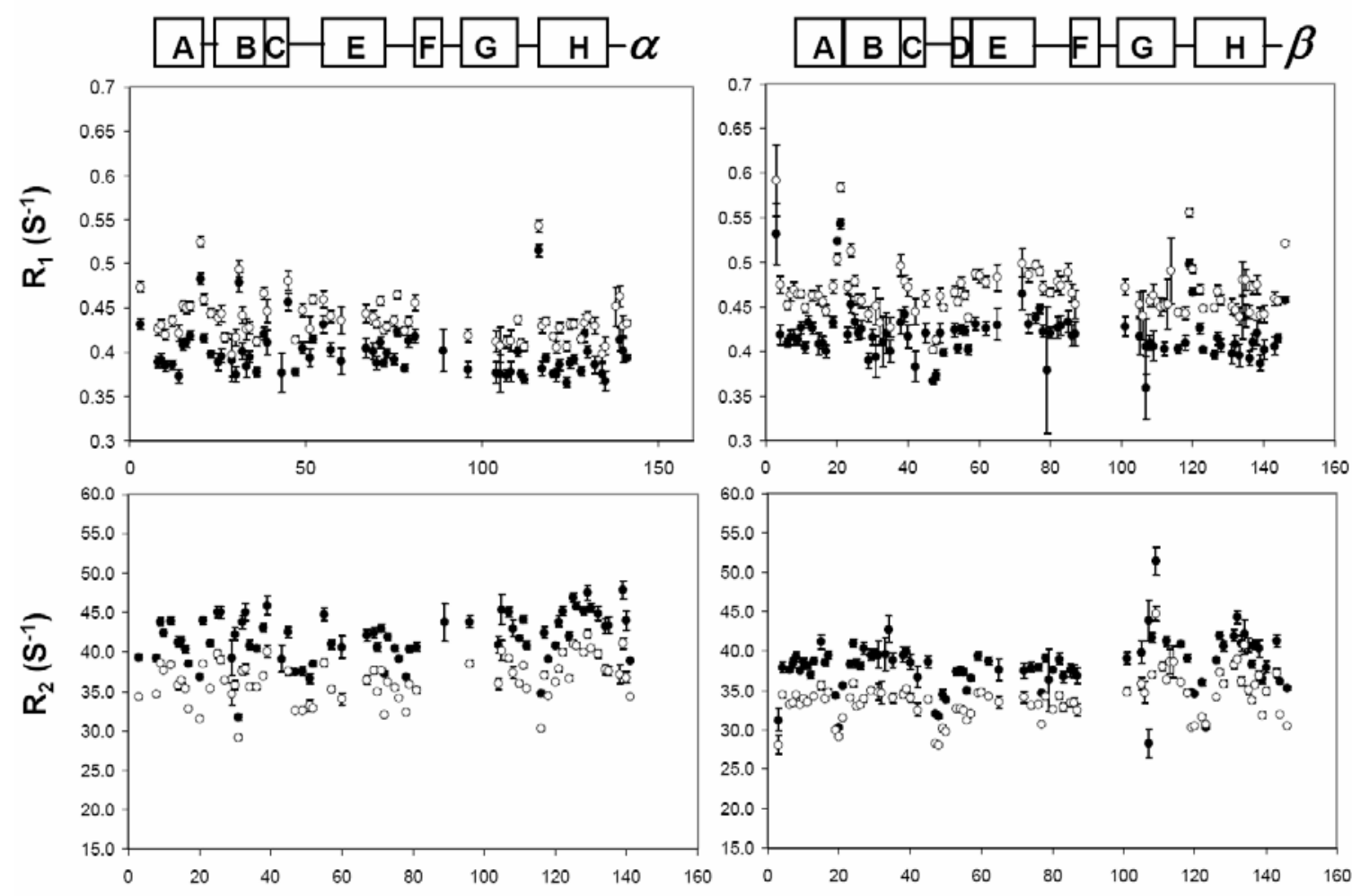

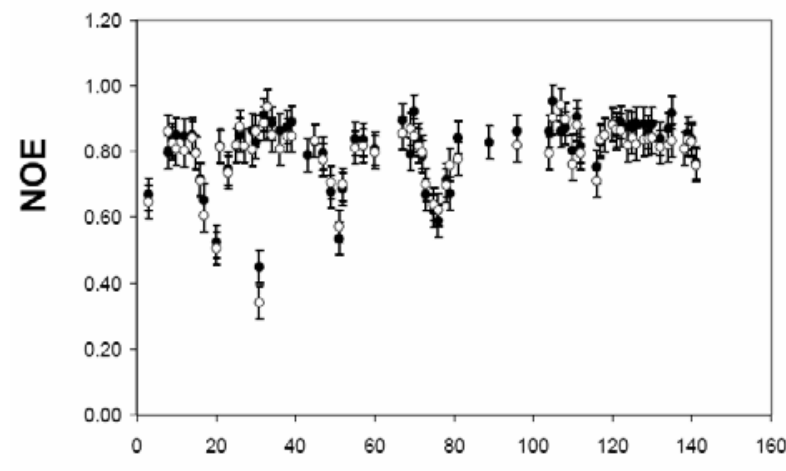

Amino Acid Residue Number

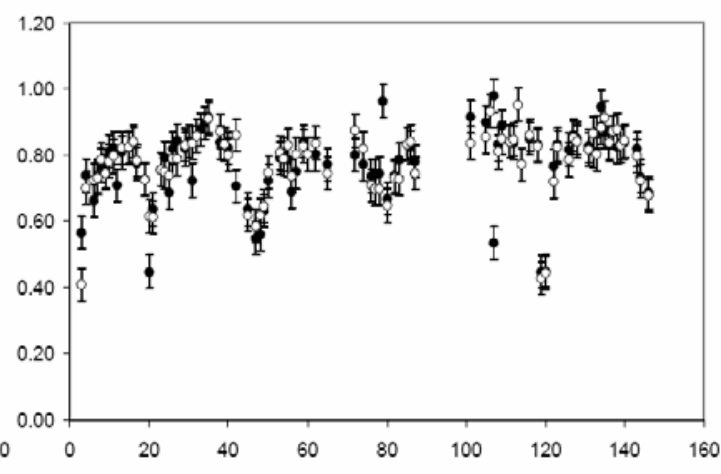

Amino Acid Residue Number 
Figure 4S (3)
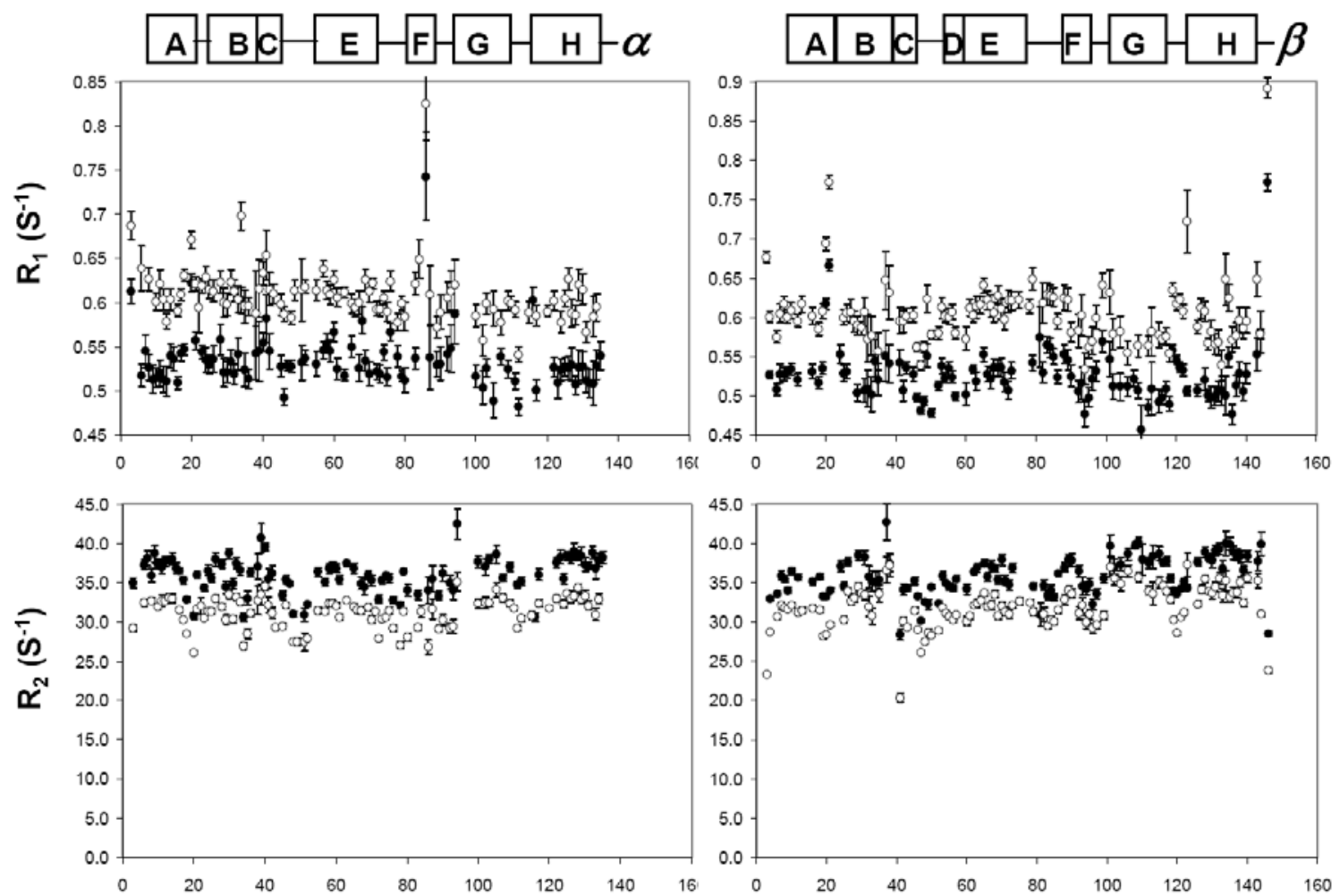

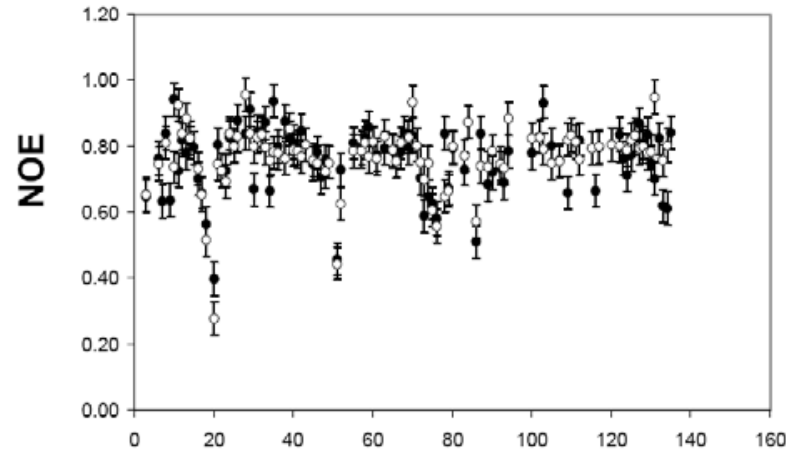

Amino Acid Residue Number

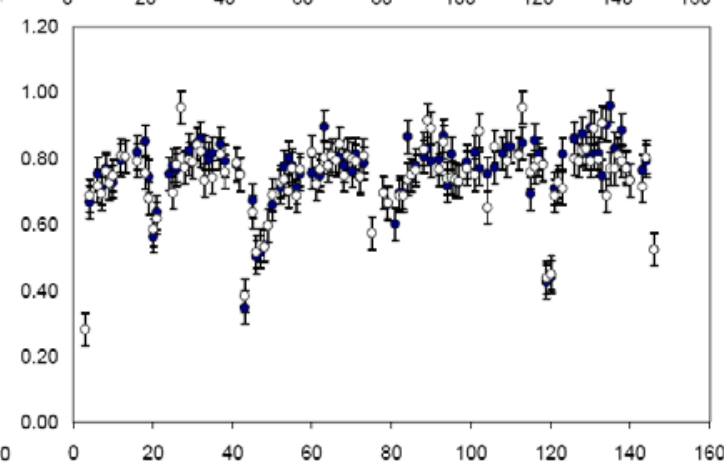

Amino Acid Residue Number 
Figure 4S (4)
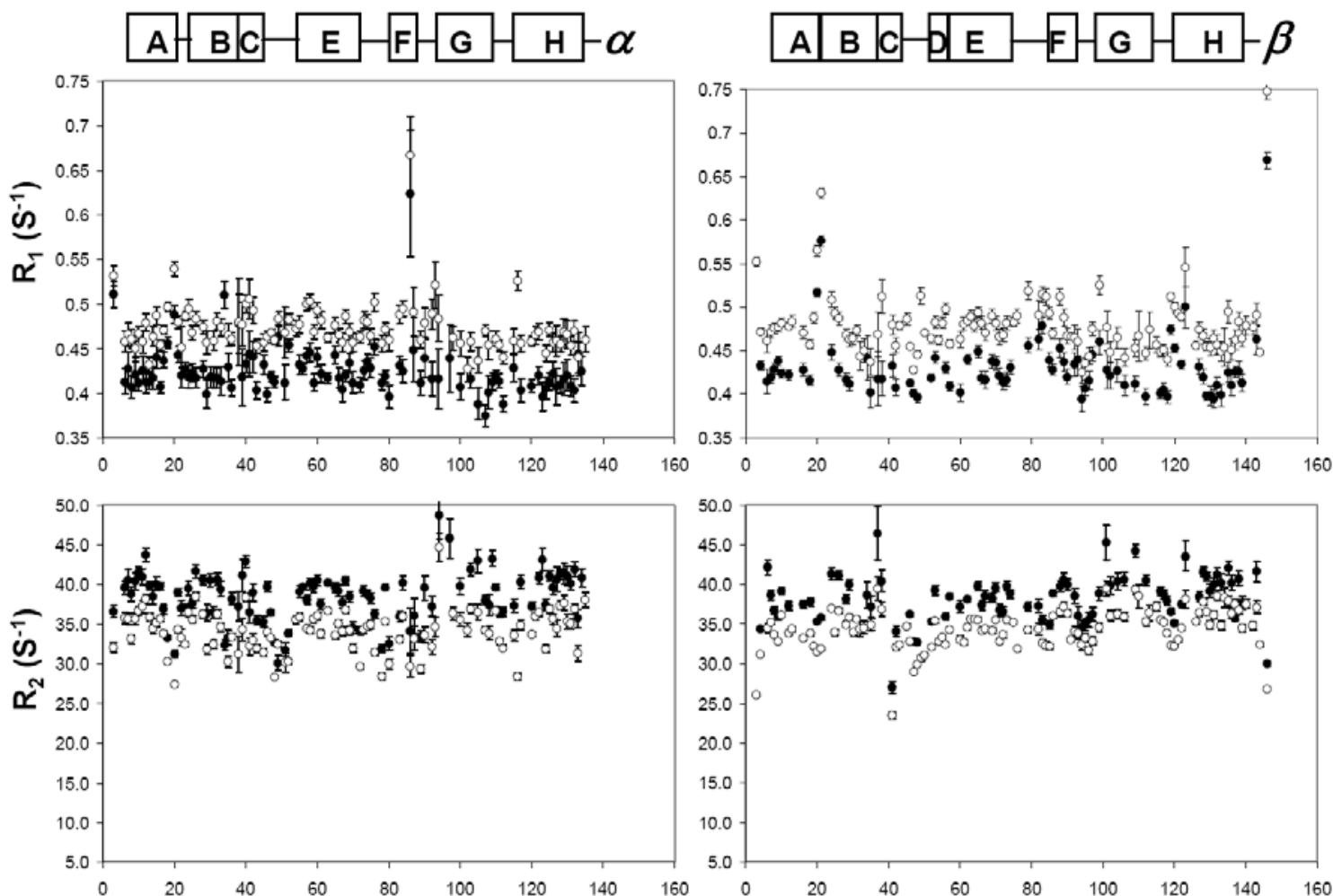

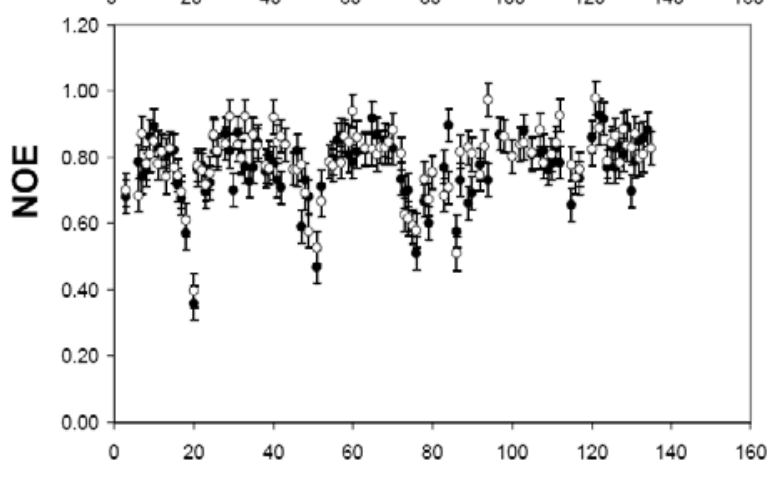

Amino Acid Residue Number

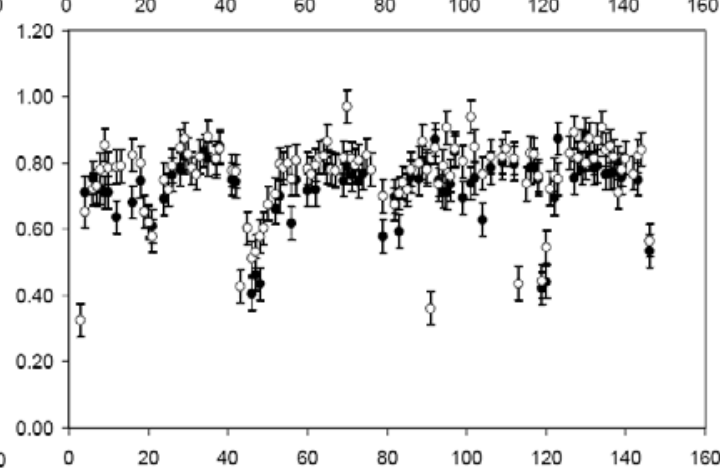

Amino Acid Residue Number 
Figure 5S
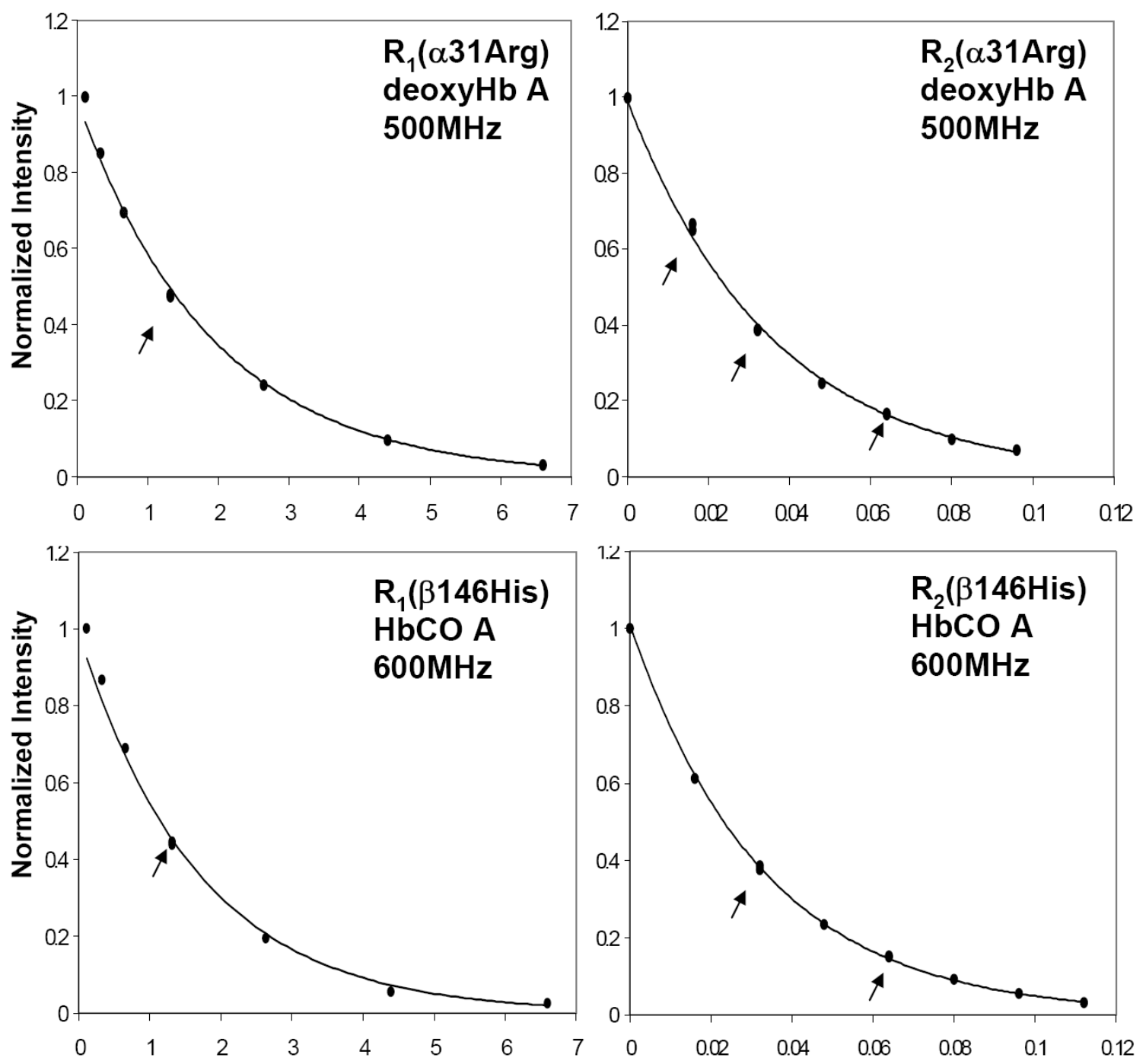

Time (sec) 
Table 1S. NMR Relaxation Parameters of Backbone Amide ${ }^{15} \mathrm{~N}$ in Deoxy-Hb A. Errors in relaxation rates were estimated by Monte Carlo procedure when fitting the exponential decays, while errors for the NOE were estimated based on the signal-to-noise of the spectra as reported by NMRPipe.

Units: $\mathrm{R}_{1}, \mathrm{~s}^{-1} ; \mathrm{R}_{2}: \mathrm{s}^{-1}$; NOE : none

$\alpha$-chain at $29{ }^{\circ} \mathrm{C}$ and at $11.7 \mathrm{~T}$ in the deoxy form

$\begin{array}{ccccccc}\begin{array}{c}\text { Residue } \\ \text { No. }\end{array} & \mathbf{R}_{\mathbf{1}} & \mathbf{R}_{\mathbf{1}} \text { error } & \mathbf{R}_{\mathbf{2}} & \mathbf{R}_{\mathbf{2}} \text { error } & \text { NOE } & \text { NOE error } \\ 3 & 0.532 & 0.006 & 35.6 & 0.4 & 0.61 & 0.05 \\ 8 & 0.492 & 0.008 & 36.6 & 0.6 & 0.80 & 0.05 \\ 9 & 0.507 & 0.008 & 38.6 & 0.6 & 0.78 & 0.05 \\ 10 & 0.493 & 0.007 & 39.0 & 0.5 & 0.79 & 0.05 \\ 12 & 0.5 & 0.008 & 39.1 & 0.4 & 0.77 & 0.05 \\ 14 & 0.501 & 0.009 & 38.7 & 0.7 & 0.81 & 0.05 \\ 15 & 0.511 & 0.007 & 39.8 & 1.5 & 0.84 & 0.05 \\ 16 & 0.503 & 0.006 & 37.9 & 0.4 & 0.65 & 0.05 \\ 17 & 0.519 & 0.006 & 35.2 & 0.3 & 0.62 & 0.05 \\ 20 & 0.577 & 0.006 & 33.3 & 0.3 & 0.48 & 0.05 \\ 21 & 0.516 & 0.006 & 40.0 & 0.4 & 0.79 & 0.05 \\ 23 & 0.497 & 0.005 & 37.0 & 0.3 & 0.72 & 0.05 \\ 25 & 0.514 & 0.008 & 41.0 & 0.7 & 0.81 & 0.05 \\ 26 & 0.497 & 0.009 & 41.1 & 0.6 & 0.83 & 0.05 \\ 29 & 0.491 & 0.022 & 39.1 & 1.7 & 0.94 & 0.05 \\ 30 & 0.486 & 0.01 & 38.4 & 0.7 & 0.82 & 0.05 \\ 31 & 0.582 & 0.012 & 28.7 & 0.5 & 0.64 & 0.05 \\ 33 & 0.491 & 0.012 & 38.6 & 0.9 & 0.89 & 0.05 \\ 34 & 0.493 & 0.008 & 37.6 & 0.6 & 0.80 & 0.05 \\ 36 & 0.481 & 0.005 & 38.1 & 0.4 & 0.78 & 0.05 \\ 38 & 0.533 & 0.007 & 38.1 & 0.4 & 0.81 & 0.05 \\ 39 & 0.515 & 0.016 & 41.0 & 1.1 & 0.83 & 0.05 \\ 15 & 0.561 & 0.009 & 39.8 & 0.6 & 0.79 & 0.05\end{array}$




\begin{tabular}{|c|c|c|c|c|c|c|}
\hline 47 & 0.48 & 0.004 & 34.8 & 0.3 & 0.71 & 0.05 \\
\hline 49 & 0.502 & 0.008 & 38.1 & 2.0 & 0.64 & 0.05 \\
\hline 51 & 0.485 & 0.011 & 34.1 & 0.6 & 0.51 & 0.05 \\
\hline 52 & 0.521 & 0.006 & 34.6 & 0.3 & 0.65 & 0.05 \\
\hline 55 & 0.515 & 0.011 & 40.0 & 0.7 & 0.78 & 0.05 \\
\hline 57 & 0.508 & 0.007 & 37.8 & 0.5 & 0.84 & 0.05 \\
\hline 60 & 0.484 & 0.013 & 38.5 & 1.0 & 0.79 & 0.05 \\
\hline 67 & 0.52 & 0.01 & 38.8 & 0.7 & 0.83 & 0.05 \\
\hline 69 & 0.48 & 0.02 & 39.5 & 1.6 & 0.73 & 0.05 \\
\hline 69 & 0.507 & 0.009 & 38.4 & 0.6 & 0.84 & 0.05 \\
\hline 70 & 0.507 & 0.007 & 37.2 & 0.5 & 0.80 & 0.05 \\
\hline 71 & 0.515 & 0.008 & 39.3 & 0.5 & 0.78 & 0.05 \\
\hline 72 & 0.491 & 0.005 & 34.1 & 0.3 & 0.76 & 0.05 \\
\hline 73 & 0.484 & 0.006 & 38.7 & 0.4 & 0.66 & 0.05 \\
\hline 75 & 0.497 & 0.007 & 36.6 & 0.4 & 0.57 & 0.05 \\
\hline 76 & 0.522 & 0.004 & 35.3 & 0.2 & 0.58 & 0.05 \\
\hline 78 & 0.487 & 0.004 & 33.1 & 0.3 & 0.67 & 0.05 \\
\hline 81 & 0.525 & 0.008 & 38.0 & 0.6 & 0.79 & 0.05 \\
\hline 96 & 0.477 & 0.008 & 40.5 & 0.6 & 0.80 & 0.05 \\
\hline 104 & 0.499 & 0.011 & 38.2 & 0.9 & 0.84 & 0.05 \\
\hline 105 & 0.49 & 0.02 & 40.9 & 1.6 & 0.77 & 0.05 \\
\hline 108 & 0.468 & 0.014 & 39.7 & 1.1 & 0.84 & 0.05 \\
\hline 110 & 0.495 & 0.006 & 37.7 & 0.4 & 0.71 & 0.05 \\
\hline 111 & 0.478 & 0.006 & 39.3 & 0.5 & 0.82 & 0.05 \\
\hline 112 & 0.47 & 0.006 & 37.4 & 0.4 & 0.73 & 0.05 \\
\hline 116 & 0.624 & 0.009 & 41.1 & 0.4 & 0.65 & 0.05 \\
\hline 117 & 0.488 & 0.008 & 39.4 & 0.6 & 0.80 & 0.05 \\
\hline 118 & 0.5 & 0.004 & 35.3 & 0.3 & 0.80 & 0.05 \\
\hline 120 & 0.474 & 0.005 & 37.2 & 0.4 & 0.78 & 0.05 \\
\hline 121 & 0.485 & 0.009 & 39.2 & 0.7 & 0.83 & 0.05 \\
\hline 122 & 0.491 & 0.007 & 40.5 & 0.6 & 0.83 & 0.05 \\
\hline
\end{tabular}




$\begin{array}{lllllll}124 & 0.471 & 0.007 & 38.6 & 0.5 & 0.78 & 0.05 \\ 125 & 0.498 & 0.007 & 41.6 & 0.5 & 0.81 & 0.05 \\ 126 & 0.496 & 0.006 & 40.6 & 0.5 & 0.85 & 0.05 \\ 128 & 0.48 & 0.006 & 41.2 & 0.5 & 0.82 & 0.05 \\ 129 & 0.506 & 0.011 & 41.9 & 0.8 & 0.88 & 0.05 \\ 130 & 0.51 & 0.007 & 41.5 & 0.6 & 0.78 & 0.05 \\ 132 & 0.493 & 0.01 & 40.1 & 0.7 & 0.82 & 0.05 \\ 134 & 0.476 & 0.009 & 38.2 & 0.7 & 0.78 & 0.05 \\ 135 & 0.48 & 0.011 & 36.4 & 0.3 & 0.77 & 0.05 \\ 139 & 0.503 & 0.01 & 38.2 & 0.7 & 0.71 & 0.05 \\ 140 & 0.506 & 0.013 & 39.3 & 1.0 & 0.78 & 0.05 \\ 141 & 0.499 & 0.004 & 36.0 & 0.2 & 0.71 & 0.05\end{array}$


$\alpha$-chain at $29{ }^{\circ} \mathrm{C}$ and at $14.1 \mathrm{~T}$ in the deoxy form

\begin{tabular}{|c|c|c|c|c|c|c|}
\hline $\begin{array}{c}\text { Residue } \\
\text { No. }\end{array}$ & $\mathbf{R}_{1}$ & $R_{1}$ error & $\mathbf{R}_{\mathbf{2}}$ & $\mathbf{R}_{\mathbf{2}}$ error & NOE & NOE error \\
\hline 3 & 0.432 & 0.006 & 39.3 & 0.4 & 0.67 & 0.05 \\
\hline 8 & 0.389 & 0.006 & 39.2 & 0.4 & 0.80 & 0.05 \\
\hline 9 & 0.392 & 0.006 & 43.8 & 0.5 & 0.84 & 0.05 \\
\hline 10 & 0.385 & 0.006 & 42.4 & 0.5 & 0.85 & 0.05 \\
\hline 12 & 0.386 & 0.005 & 43.9 & 0.5 & 0.85 & 0.05 \\
\hline 14 & 0.373 & 0.008 & 41.2 & 0.7 & 0.85 & 0.05 \\
\hline 15 & 0.409 & 0.006 & 41.4 & 0.5 & 0.80 & 0.05 \\
\hline 16 & 0.411 & 0.005 & 40.3 & 0.4 & 0.72 & 0.05 \\
\hline 17 & 0.419 & 0.005 & 38.5 & 0.3 & 0.65 & 0.05 \\
\hline 20 & 0.483 & 0.006 & 36.9 & 0.3 & 0.52 & 0.05 \\
\hline 21 & 0.416 & 0.005 & 44.0 & 0.5 & 0.82 & 0.05 \\
\hline 23 & 0.398 & 0.004 & 41.1 & 0.3 & 0.75 & 0.05 \\
\hline 25 & 0.389 & 0.009 & 45.0 & 0.8 & 0.82 & 0.05 \\
\hline 26 & 0.396 & 0.008 & 45.0 & 0.8 & 0.85 & 0.05 \\
\hline 29 & 0.391 & 0.024 & 39.2 & 2.2 & 0.86 & 0.05 \\
\hline 30 & 0.375 & 0.009 & 42.3 & 0.8 & 0.83 & 0.05 \\
\hline 31 & 0.479 & 0.011 & 31.7 & 0.4 & 0.45 & 0.05 \\
\hline 32 & 0.401 & 0.009 & 43.8 & 0.8 & 0.91 & 0.05 \\
\hline 33 & 0.384 & 0.013 & 45.0 & 1.1 & 0.89 & 0.05 \\
\hline 34 & 0.395 & 0.007 & 40.8 & 0.6 & 0.89 & 0.05 \\
\hline 36 & 0.378 & 0.005 & 40.5 & 0.4 & 0.86 & 0.05 \\
\hline 38 & 0.421 & 0.007 & 43.0 & 0.5 & 0.88 & 0.05 \\
\hline 39 & 0.411 & 0.014 & 45.9 & 1.2 & 0.89 & 0.05 \\
\hline 43 & 0.377 & 0.022 & 39.1 & 1.7 & 0.79 & 0.05 \\
\hline 45 & 0.457 & 0.01 & 42.5 & 0.7 & 0.83 & 0.05 \\
\hline 47 & 0.378 & 0.004 & 37.5 & 0.3 & 0.80 & 0.05 \\
\hline 49 & 0.405 & 0.007 & 37.5 & 0.5 & 0.68 & 0.05 \\
\hline 51 & 0.394 & 0.01 & 36.6 & 0.6 & 0.54 & 0.05 \\
\hline
\end{tabular}




\begin{tabular}{|c|c|c|c|c|c|c|}
\hline 52 & 0.415 & 0.005 & 38.5 & 0.4 & 0.69 & 0.05 \\
\hline 55 & 0.432 & 0.01 & 44.7 & 0.9 & 0.84 & 0.05 \\
\hline 57 & 0.403 & 0.007 & 41.0 & 0.6 & 0.84 & 0.05 \\
\hline 60 & 0.39 & 0.015 & 40.6 & 1.4 & 0.81 & 0.05 \\
\hline 67 & 0.405 & 0.011 & 42.1 & 0.8 & 0.89 & 0.05 \\
\hline 69 & 0.401 & 0.009 & 42.4 & 0.7 & 0.79 & 0.05 \\
\hline 70 & 0.388 & 0.007 & 40.6 & 0.5 & 0.92 & 0.05 \\
\hline 71 & 0.411 & 0.006 & 43.0 & 0.5 & 0.84 & 0.05 \\
\hline 72 & 0.388 & 0.004 & 37.2 & 0.3 & 0.79 & 0.05 \\
\hline 73 & 0.399 & 0.005 & 41.9 & 0.5 & 0.67 & 0.05 \\
\hline 75 & 0.392 & 0.006 & 40.5 & 0.4 & 0.62 & 0.05 \\
\hline 76 & 0.423 & 0.004 & 39.1 & 0.3 & 0.59 & 0.05 \\
\hline 78 & 0.382 & 0.004 & 36.8 & 0.3 & 0.72 & 0.05 \\
\hline 79 & 0.413 & 0.007 & 40.4 & 0.5 & 0.67 & 0.05 \\
\hline 81 & 0.418 & 0.008 & 40.6 & 0.6 & 0.84 & 0.05 \\
\hline 89 & 0.402 & 0.024 & 43.8 & 2.4 & 0.83 & 0.05 \\
\hline 96 & 0.381 & 0.009 & 43.8 & 0.7 & 0.86 & 0.05 \\
\hline 104 & 0.377 & 0.012 & 40.9 & 1.1 & 0.86 & 0.05 \\
\hline 105 & 0.376 & 0.021 & 45.4 & 1.9 & 0.95 & 0.05 \\
\hline 107 & 0.374 & 0.007 & 45.0 & 0.6 & 0.86 & 0.05 \\
\hline 108 & 0.378 & 0.011 & 43.0 & 1.1 & 0.87 & 0.05 \\
\hline 110 & 0.401 & 0.005 & 41.7 & 0.4 & 0.80 & 0.05 \\
\hline 111 & 0.376 & 0.005 & 44.1 & 0.5 & 0.91 & 0.05 \\
\hline 112 & 0.37 & 0.005 & 40.8 & 0.5 & 0.82 & 0.05 \\
\hline 116 & 0.515 & 0.007 & 34.7 & 0.4 & 0.75 & 0.05 \\
\hline 117 & 0.382 & 0.008 & 42.5 & 0.7 & 0.83 & 0.05 \\
\hline 118 & 0.394 & 0.004 & 39.1 & 0.3 & 0.85 & 0.05 \\
\hline 120 & 0.376 & 0.004 & 40.8 & 0.3 & 0.88 & 0.05 \\
\hline 121 & 0.375 & 0.007 & 43.8 & 0.7 & 0.86 & 0.05 \\
\hline 122 & 0.386 & 0.006 & 45.2 & 0.6 & 0.89 & 0.05 \\
\hline 124 & 0.366 & 0.006 & 42.0 & 0.5 & 0.88 & 0.05 \\
\hline
\end{tabular}




$\begin{array}{lllllll}125 & 0.388 & 0.006 & 46.9 & 0.5 & 0.87 & 0.05 \\ 126 & 0.392 & 0.005 & 45.8 & 0.5 & 0.89 & 0.05 \\ 128 & 0.379 & 0.005 & 45.2 & 0.5 & 0.88 & 0.05 \\ 129 & 0.421 & 0.01 & 47.5 & 0.9 & 0.86 & 0.05 \\ 130 & 0.401 & 0.007 & 45.6 & 0.6 & 0.88 & 0.05 \\ 132 & 0.386 & 0.01 & 44.9 & 0.9 & 0.84 & 0.05 \\ 134 & 0.376 & 0.011 & 43.3 & 0.9 & 0.87 & 0.05 \\ 135 & 0.368 & 0.011 & 43.4 & 1.0 & 0.92 & 0.05 \\ 139 & 0.414 & 0.013 & 47.9 & 1.2 & 0.86 & 0.05 \\ 140 & 0.402 & 0.014 & 44.0 & 1.2 & 0.84 & 0.05 \\ 141 & 0.394 & 0.003 & 38.9 & 0.2 & 0.77 & 0.05\end{array}$


$\alpha$-chain at $34^{\circ} \mathrm{C}$ and at $11.7 \mathrm{~T}$ in the deoxy form

\begin{tabular}{|c|c|c|c|c|c|c|}
\hline $\begin{array}{c}\text { Residue } \\
\text { No. }\end{array}$ & $\mathbf{R}_{1}$ & $\mathbf{R}_{\mathbf{1}}$ error & $\mathbf{R}_{\mathbf{2}}$ & $\mathbf{R}_{\mathbf{2}}$ error & NOE & NOE error \\
\hline 3 & 0.598 & 0.006 & 26.3 & 0.2 & 0.63 & 0.05 \\
\hline 8 & 0.557 & 0.006 & 28.2 & 0.3 & 0.79 & 0.05 \\
\hline 9 & 0.568 & 0.007 & 30.5 & 0.3 & 0.82 & 0.05 \\
\hline 10 & 0.563 & 0.006 & 30.6 & 0.3 & 0.79 & 0.05 \\
\hline 12 & 0.565 & 0.006 & 30.9 & 0.3 & 0.79 & 0.05 \\
\hline 14 & 0.568 & 0.008 & 30.1 & 0.4 & 0.79 & 0.05 \\
\hline 15 & 0.566 & 0.016 & 29.8 & 0.3 & 0.76 & 0.05 \\
\hline 16 & 0.569 & 0.005 & 28.2 & 0.2 & 0.69 & 0.05 \\
\hline 17 & 0.574 & 0.005 & 26.3 & 0.2 & 0.56 & 0.05 \\
\hline 20 & 0.654 & 0.006 & 23.9 & 0.2 & 0.45 & 0.05 \\
\hline 21 & 0.6 & 0.005 & 30.9 & 0.2 & 0.76 & 0.05 \\
\hline 23 & 0.567 & 0.005 & 27.7 & 0.2 & 0.72 & 0.05 \\
\hline 25 & 0.584 & 0.007 & 31.0 & 0.3 & 0.76 & 0.05 \\
\hline 26 & 0.561 & 0.007 & 32.3 & 0.3 & 0.80 & 0.05 \\
\hline 27 & 0.546 & 0.005 & 30.1 & 0.2 & 0.80 & 0.05 \\
\hline 29 & 0.542 & 0.017 & 28.8 & 0.8 & 0.85 & 0.05 \\
\hline 30 & 0.543 & 0.008 & 29.3 & 0.4 & 0.79 & 0.05 \\
\hline 31 & 0.646 & 0.008 & 21.5 & 0.2 & 0.43 & 0.05 \\
\hline 32 & 0.571 & 0.009 & 32.0 & 0.4 & 0.93 & 0.05 \\
\hline 33 & 0.577 & 0.01 & 31.4 & 0.5 & 0.85 & 0.05 \\
\hline 34 & 0.565 & 0.007 & 29.2 & 0.3 & 0.81 & 0.05 \\
\hline 36 & 0.552 & 0.005 & 29.6 & 0.2 & 0.79 & 0.05 \\
\hline 38 & 0.615 & 0.006 & 29.6 & 0.2 & 0.81 & 0.05 \\
\hline 39 & 0.606 & 0.013 & 33.4 & 0.6 & 0.80 & 0.05 \\
\hline 43 & 0.559 & 0.013 & 27.4 & 0.6 & 0.75 & 0.05 \\
\hline 45 & 0.639 & 0.008 & 30.0 & 0.3 & 0.82 & 0.05 \\
\hline 47 & 0.538 & 0.004 & 26.2 & 0.2 & 0.72 & 0.05 \\
\hline 49 & 0.57 & 0.007 & 25.7 & 0.3 & 0.67 & 0.05 \\
\hline
\end{tabular}




\begin{tabular}{|c|c|c|c|c|c|c|}
\hline 51 & 0.539 & 0.015 & 24.1 & 0.6 & 0.59 & 0.05 \\
\hline 52 & 0.583 & 0.005 & 25.3 & 0.2 & 0.65 & 0.05 \\
\hline 55 & 0.595 & 0.009 & 31.4 & 0.4 & 0.78 & 0.05 \\
\hline 57 & 0.577 & 0.006 & 29.0 & 0.3 & 0.79 & 0.05 \\
\hline 60 & 0.564 & 0.01 & 27.8 & 0.4 & 0.77 & 0.05 \\
\hline 67 & 0.578 & 0.009 & 29.8 & 0.4 & 0.82 & 0.05 \\
\hline 69 & 0.577 & 0.007 & 30.2 & 0.3 & 0.83 & 0.05 \\
\hline 70 & 0.575 & 0.006 & 28.3 & 0.3 & 0.80 & 0.05 \\
\hline 72 & 0.561 & 0.004 & 25.0 & 0.2 & 0.76 & 0.05 \\
\hline 73 & 0.56 & 0.005 & 28.8 & 0.2 & 0.67 & 0.05 \\
\hline 75 & 0.556 & 0.006 & 27.8 & 0.2 & 0.64 & 0.05 \\
\hline 76 & 0.593 & 0.004 & 25.8 & 0.1 & 0.61 & 0.05 \\
\hline 78 & 0.543 & 0.004 & 23.6 & 0.1 & 0.70 & 0.05 \\
\hline 79 & 0.58 & 0.009 & 27.5 & 0.2 & 0.51 & 0.05 \\
\hline 81 & 0.588 & 0.007 & 28.9 & 0.3 & 0.80 & 0.05 \\
\hline 89 & 0.547 & 0.014 & 30.1 & 0.7 & 0.76 & 0.05 \\
\hline 96 & 0.546 & 0.006 & 31.7 & 0.3 & 0.79 & 0.05 \\
\hline 104 & 0.559 & 0.01 & 29.0 & 0.5 & 0.84 & 0.05 \\
\hline 105 & 0.544 & 0.016 & 34.3 & 0.9 & 0.78 & 0.05 \\
\hline 107 & 0.549 & 0.007 & 33.2 & 0.4 & 0.91 & 0.05 \\
\hline 108 & 0.553 & 0.013 & 29.9 & 0.6 & 0.83 & 0.05 \\
\hline 110 & 0.568 & 0.006 & 28.8 & 0.2 & 0.75 & 0.05 \\
\hline 111 & 0.55 & 0.005 & 30.7 & 0.3 & 0.81 & 0.05 \\
\hline 112 & 0.529 & 0.005 & 28.2 & 0.2 & 0.76 & 0.05 \\
\hline 116 & 0.69 & 0.008 & 21.9 & 0.2 & 0.66 & 0.05 \\
\hline 117 & 0.555 & 0.007 & 30.3 & 0.3 & 0.79 & 0.05 \\
\hline 118 & 0.573 & 0.004 & 27.5 & 0.2 & 0.78 & 0.05 \\
\hline 120 & 0.551 & 0.004 & 29.0 & 0.2 & 0.82 & 0.05 \\
\hline 121 & 0.549 & 0.007 & 30.7 & 0.4 & 0.81 & 0.05 \\
\hline 122 & 0.563 & 0.007 & 33.1 & 0.3 & 0.82 & 0.05 \\
\hline 124 & 0.55 & 0.006 & 30.6 & 0.3 & 0.78 & 0.05 \\
\hline
\end{tabular}




$\begin{array}{lllllll}125 & 0.571 & 0.006 & 33.0 & 0.3 & 0.82 & 0.05 \\ 126 & 0.568 & 0.006 & 32.4 & 0.3 & 0.82 & 0.05 \\ 128 & 0.551 & 0.005 & 33.2 & 0.3 & 0.80 & 0.05 \\ 129 & 0.589 & 0.009 & 33.3 & 0.5 & 0.83 & 0.05 \\ 130 & 0.575 & 0.006 & 33.3 & 0.3 & 0.80 & 0.05 \\ 132 & 0.557 & 0.008 & 32.1 & 0.4 & 0.82 & 0.05 \\ 134 & 0.548 & 0.008 & 30.3 & 0.4 & 0.79 & 0.05 \\ 135 & 0.533 & 0.009 & 30.6 & 0.4 & 0.83 & 0.05 \\ 138 & 0.588 & 0.016 & 30.1 & 0.7 & 0.85 & 0.05 \\ 139 & 0.585 & 0.011 & 31.3 & 0.5 & 0.77 & 0.05 \\ 140 & 0.585 & 0.012 & 30.5 & 0.5 & 0.77 & 0.05 \\ 141 & 0.563 & 0.003 & 26.8 & 0.1 & 0.71 & 0.05\end{array}$


$\alpha$-chain at $34^{\circ} \mathrm{C}$ and at $14.1 \mathrm{~T}$ in the deoxy form

\begin{tabular}{|c|c|c|c|c|c|c|}
\hline $\begin{array}{c}\text { Residue } \\
\text { No. }\end{array}$ & $\mathbf{R}_{1}$ & $\mathbf{R}_{\mathbf{1}}$ error & $\mathbf{R}_{\mathbf{2}}$ & $\mathbf{R}_{\mathbf{2}}$ error & NOE & NOE error \\
\hline 3 & 0.474 & 0.006 & 34.3 & 0.3 & 0.65 & 0.05 \\
\hline 8 & 0.426 & 0.006 & 34.6 & 0.3 & 0.86 & 0.05 \\
\hline 9 & 0.431 & 0.006 & 38.5 & 0.4 & 0.83 & 0.05 \\
\hline 10 & 0.42 & 0.006 & 37.7 & 0.3 & 0.81 & 0.05 \\
\hline 12 & 0.436 & 0.006 & 38.4 & 0.3 & 0.80 & 0.05 \\
\hline 14 & 0.422 & 0.009 & 35.7 & 0.4 & 0.84 & 0.05 \\
\hline 15 & 0.453 & 0.006 & 36.4 & 0.3 & 0.79 & 0.05 \\
\hline 16 & 0.45 & 0.006 & 35.4 & 0.3 & 0.71 & 0.05 \\
\hline 17 & 0.452 & 0.005 & 32.8 & 0.2 & 0.61 & 0.05 \\
\hline 20 & 0.525 & 0.006 & 31.6 & 0.2 & 0.51 & 0.05 \\
\hline 21 & 0.46 & 0.006 & 38.5 & 0.3 & 0.82 & 0.05 \\
\hline 23 & 0.444 & 0.005 & 35.3 & 0.2 & 0.74 & 0.05 \\
\hline 25 & 0.439 & 0.008 & 39.7 & 0.5 & 0.82 & 0.05 \\
\hline 26 & 0.444 & 0.009 & 39.1 & 0.5 & 0.88 & 0.05 \\
\hline 27 & 0.417 & 0.005 & 36.4 & 0.3 & 0.82 & 0.05 \\
\hline 29 & 0.397 & 0.024 & 34.7 & 1.4 & 0.81 & 0.05 \\
\hline 30 & 0.417 & 0.009 & 35.8 & 0.5 & 0.86 & 0.05 \\
\hline 31 & 0.494 & 0.009 & 29.2 & 0.3 & 0.34 & 0.05 \\
\hline 32 & 0.442 & 0.009 & 37.6 & 0.5 & 0.89 & 0.05 \\
\hline 33 & 0.426 & 0.012 & 37.9 & 0.7 & 0.94 & 0.05 \\
\hline 34 & 0.428 & 0.007 & 35.6 & 0.4 & 0.85 & 0.05 \\
\hline 36 & 0.412 & 0.005 & 35.5 & 0.3 & 0.81 & 0.05 \\
\hline 38 & 0.467 & 0.007 & 37.0 & 0.3 & 0.85 & 0.05 \\
\hline 39 & 0.447 & 0.013 & 40.1 & 0.7 & 0.85 & 0.05 \\
\hline 45 & 0.481 & 0.01 & 37.5 & 0.5 & 0.83 & 0.05 \\
\hline 47 & 0.414 & 0.004 & 32.5 & 0.2 & 0.78 & 0.05 \\
\hline 49 & 0.448 & 0.007 & 32.6 & 0.3 & 0.71 & 0.05 \\
\hline 51 & 0.426 & 0.015 & 33.2 & 0.7 & 0.57 & 0.05 \\
\hline
\end{tabular}




\begin{tabular}{|c|c|c|c|c|c|c|}
\hline 52 & 0.46 & 0.005 & 32.9 & 0.2 & 0.70 & 0.05 \\
\hline 55 & 0.46 & 0.01 & 38.6 & 0.5 & 0.81 & 0.05 \\
\hline 57 & 0.441 & 0.007 & 35.3 & 0.4 & 0.82 & 0.05 \\
\hline 60 & 0.436 & 0.015 & 34.0 & 0.7 & 0.80 & 0.05 \\
\hline 67 & 0.444 & 0.01 & 36.5 & 0.5 & 0.86 & 0.05 \\
\hline 69 & 0.44 & 0.008 & 37.7 & 0.4 & 0.87 & 0.05 \\
\hline 70 & 0.433 & 0.006 & 34.9 & 0.3 & 0.85 & 0.05 \\
\hline 71 & 0.458 & 0.005 & 37.7 & 0.3 & 0.82 & 0.05 \\
\hline 72 & 0.425 & 0.004 & 32.0 & 0.2 & 0.80 & 0.05 \\
\hline 73 & 0.429 & 0.006 & 36.2 & 0.3 & 0.70 & 0.05 \\
\hline 75 & 0.436 & 0.006 & 35.5 & 0.3 & 0.64 & 0.05 \\
\hline 76 & 0.465 & 0.004 & 34.2 & 0.2 & 0.62 & 0.05 \\
\hline 78 & 0.424 & 0.004 & 32.3 & 0.2 & 0.70 & 0.05 \\
\hline 79 & 0.435 & 0.006 & 35.9 & 0.3 & 0.76 & 0.05 \\
\hline 81 & 0.456 & 0.009 & 35.1 & 0.4 & 0.78 & 0.05 \\
\hline 96 & 0.419 & 0.007 & 38.5 & 0.4 & 0.82 & 0.05 \\
\hline 104 & 0.412 & 0.012 & 36.0 & 0.7 & 0.80 & 0.05 \\
\hline 105 & 0.408 & 0.02 & 40.2 & 1.2 & 0.90 & 0.05 \\
\hline 107 & 0.412 & 0.007 & 39.2 & 0.4 & 0.94 & 0.05 \\
\hline 108 & 0.413 & 0.012 & 37.3 & 0.7 & 0.90 & 0.05 \\
\hline 110 & 0.437 & 0.005 & 36.0 & 0.3 & 0.76 & 0.05 \\
\hline 111 & 0.41 & 0.005 & 38.3 & 0.3 & 0.88 & 0.05 \\
\hline 112 & 0.407 & 0.005 & 35.4 & 0.3 & 0.80 & 0.05 \\
\hline 116 & 0.543 & 0.007 & 30.2 & 0.2 & 0.71 & 0.05 \\
\hline 117 & 0.43 & 0.008 & 37.0 & 0.4 & 0.84 & 0.05 \\
\hline 118 & 0.435 & 0.004 & 34.5 & 0.2 & 0.85 & 0.05 \\
\hline 120 & 0.418 & 0.004 & 36.2 & 0.2 & 0.88 & 0.05 \\
\hline 121 & 0.406 & 0.007 & 37.8 & 0.4 & 0.87 & 0.05 \\
\hline 122 & 0.428 & 0.006 & 40.0 & 0.4 & 0.86 & 0.05 \\
\hline 124 & 0.407 & 0.006 & 36.6 & 0.3 & 0.82 & 0.05 \\
\hline 125 & 0.432 & 0.006 & 41.0 & 0.4 & 0.84 & 0.05 \\
\hline
\end{tabular}




$\begin{array}{lllllll}126 & 0.432 & 0.005 & 40.7 & 0.3 & 0.82 & 0.05 \\ 128 & 0.415 & 0.005 & 39.9 & 0.3 & 0.84 & 0.05 \\ 129 & 0.434 & 0.009 & 42.3 & 0.6 & 0.84 & 0.05 \\ 130 & 0.44 & 0.006 & 40.5 & 0.4 & 0.84 & 0.05 \\ 132 & 0.43 & 0.009 & 39.7 & 0.5 & 0.82 & 0.05 \\ 134 & 0.398 & 0.009 & 37.7 & 0.6 & 0.82 & 0.05 \\ 135 & 0.407 & 0.01 & 37.5 & 0.6 & 0.83 & 0.05 \\ 138 & 0.452 & 0.022 & 37.2 & 1.2 & 0.81 & 0.05 \\ 139 & 0.463 & 0.012 & 41.0 & 0.7 & 0.84 & 0.05 \\ 140 & 0.43 & 0.012 & 36.8 & 0.7 & 0.83 & 0.05 \\ 141 & 0.433 & 0.003 & 34.3 & 0.2 & 0.76 & 0.05\end{array}$


$\beta$-chain at $29{ }^{\circ} \mathrm{C}$ and at $11.7 \mathrm{~T}$ in the deoxy form

\begin{tabular}{|c|c|c|c|c|c|c|}
\hline $\begin{array}{c}\text { Residue } \\
\text { No. }\end{array}$ & $\mathbf{R}_{1}$ & $R_{1}$ error & $\mathbf{R}_{\mathbf{2}}$ & $\mathbf{R}_{\mathbf{2}}$ error & NOE & NOE error \\
\hline 3 & 0.637 & 0.044 & 33.9 & 2.5 & 0.57 & 0.05 \\
\hline 4 & 0.532 & 0.014 & 35.2 & 0.7 & 0.68 & 0.05 \\
\hline 6 & 0.509 & 0.007 & 35.6 & 0.3 & 0.68 & 0.05 \\
\hline 7 & 0.537 & 0.013 & 36.3 & 0.6 & 0.71 & 0.05 \\
\hline 8 & 0.531 & 0.012 & 36.4 & 0.6 & 0.81 & 0.05 \\
\hline 9 & 0.525 & 0.008 & 35.3 & 0.4 & 0.76 & 0.05 \\
\hline 11 & 0.516 & 0.009 & 36.6 & 0.5 & 0.78 & 0.05 \\
\hline 12 & 0.538 & 0.013 & 35.6 & 0.5 & 0.94 & 0.05 \\
\hline 13 & 0.538 & 0.01 & 34.3 & 0.5 & 0.79 & 0.05 \\
\hline 15 & 0.521 & 0.019 & 37.5 & 0.9 & 0.80 & 0.05 \\
\hline 16 & 0.527 & 0.01 & 36.6 & 0.5 & 0.79 & 0.05 \\
\hline 17 & 0.516 & 0.011 & 37.1 & 0.6 & 0.80 & 0.05 \\
\hline 19 & 0.547 & 0.007 & 31.8 & 0.3 & 0.70 & 0.05 \\
\hline 20 & 0.651 & 0.005 & 28.5 & 0.1 & 0.45 & 0.05 \\
\hline 21 & 0.67 & 0.008 & 33.2 & 0.3 & 0.60 & 0.05 \\
\hline 23 & 0.545 & 0.012 & 35.8 & 0.5 & 0.77 & 0.05 \\
\hline 24 & 0.575 & 0.012 & 37.2 & 0.5 & 0.77 & 0.05 \\
\hline 25 & 0.531 & 0.011 & 35.6 & 0.5 & 0.73 & 0.05 \\
\hline 26 & 0.54 & 0.012 & 36.3 & 0.6 & 0.81 & 0.05 \\
\hline 27 & 0.54 & 0.014 & 36.9 & 0.6 & 0.79 & 0.05 \\
\hline 29 & 0.526 & 0.013 & 37.5 & 0.7 & 0.87 & 0.05 \\
\hline 30 & 0.527 & 0.013 & 36.6 & 0.6 & 0.80 & 0.05 \\
\hline 31 & 0.523 & 0.031 & 35.5 & 1.5 & 0.75 & 0.05 \\
\hline 32 & 0.501 & 0.037 & 38.3 & 2.1 & 0.81 & 0.05 \\
\hline 34 & 0.534 & 0.032 & 36.1 & 1.5 & 0.88 & 0.05 \\
\hline 35 & 0.528 & 0.02 & 37.3 & 1.0 & 0.79 & 0.05 \\
\hline 38 & 0.554 & 0.02 & 38.4 & 1.0 & 0.85 & 0.05 \\
\hline 39 & 0.549 & 0.013 & 37.5 & 0.6 & 0.94 & 0.05 \\
\hline
\end{tabular}




\begin{tabular}{|c|c|c|c|c|c|c|}
\hline 40 & 0.521 & 0.018 & 35.6 & 0.9 & 0.75 & 0.05 \\
\hline 42 & 0.524 & 0.025 & 34.9 & 1.2 & 0.77 & 0.05 \\
\hline 45 & 0.526 & 0.014 & 37.0 & 0.7 & 0.68 & 0.05 \\
\hline 47 & 0.463 & 0.005 & 30.5 & 0.2 & 0.54 & 0.05 \\
\hline 48 & 0.475 & 0.007 & 31.5 & 0.3 & 0.61 & 0.05 \\
\hline 49 & 0.511 & 0.018 & 33.0 & 0.8 & 0.63 & 0.05 \\
\hline 50 & 0.501 & 0.006 & 31.6 & 0.2 & 0.72 & 0.05 \\
\hline 54 & 0.524 & 0.008 & 35.5 & 0.4 & 0.77 & 0.05 \\
\hline 55 & 0.536 & 0.009 & 34.8 & 0.4 & 0.79 & 0.05 \\
\hline 56 & 0.525 & 0.009 & 33.9 & 0.4 & 0.68 & 0.05 \\
\hline 57 & 0.501 & 0.007 & 35.0 & 0.4 & 0.78 & 0.05 \\
\hline 59 & 0.548 & 0.009 & 37.2 & 0.4 & 0.79 & 0.05 \\
\hline 62 & 0.539 & 0.011 & 37.0 & 0.5 & 0.81 & 0.05 \\
\hline 65 & 0.538 & 0.021 & 37.3 & 1.0 & 0.80 & 0.05 \\
\hline 72 & 0.561 & 0.022 & 36.6 & 1.0 & 0.81 & 0.05 \\
\hline 74 & 0.552 & 0.014 & 34.4 & 0.6 & 0.77 & 0.05 \\
\hline 76 & 0.553 & 0.009 & 35.4 & 0.4 & 0.73 & 0.05 \\
\hline 77 & 0.56 & 0.009 & 33.6 & 0.3 & 0.69 & 0.05 \\
\hline 78 & 0.523 & 0.012 & 36.2 & 0.5 & 0.70 & 0.05 \\
\hline 80 & 0.541 & 0.009 & 34.4 & 0.4 & 0.66 & 0.05 \\
\hline 82 & 0.541 & 0.015 & 35.9 & 0.6 & 0.73 & 0.05 \\
\hline 83 & 0.546 & 0.015 & 34.6 & 0.7 & 0.74 & 0.05 \\
\hline 85 & 0.537 & 0.017 & 34.9 & 0.8 & 0.80 & 0.05 \\
\hline 86 & 0.512 & 0.016 & 34.9 & 0.8 & 0.83 & 0.05 \\
\hline 87 & 0.532 & 0.019 & 34.4 & 0.9 & 0.76 & 0.05 \\
\hline 101 & 0.54 & 0.013 & 36.9 & 0.7 & 0.82 & 0.05 \\
\hline 105 & 0.511 & 0.022 & 37.8 & 1.2 & 0.80 & 0.05 \\
\hline 106 & 0.496 & 0.038 & 38.2 & 2.1 & 0.93 & 0.05 \\
\hline 107 & 0.5 & 0.029 & 37.3 & 1.6 & 0.90 & 0.05 \\
\hline 108 & 0.527 & 0.012 & 38.8 & 0.6 & 0.83 & 0.05 \\
\hline 109 & 0.53 & 0.021 & 44.8 & 1.3 & 0.90 & 0.05 \\
\hline
\end{tabular}




\begin{tabular}{|c|c|c|c|c|c|}
\hline 111 & 0.506 & 0.017 & 39.1 & 0.9 & 0.84 \\
\hline 112 & 0.512 & 0.011 & 39.0 & 0.6 & 0.84 \\
\hline 114 & 0.576 & 0.064 & 39.3 & 3.2 & 1.16 \\
\hline 116 & 0.524 & 0.008 & 37.4 & 0.4 & 0.85 \\
\hline 118 & 0.515 & 0.014 & 36.6 & 0.7 & 0.83 \\
\hline 119 & 0.604 & 0.008 & 33.5 & 0.3 & 0.43 \\
\hline 120 & 0.564 & 0.006 & 32.8 & 0.2 & 0.44 \\
\hline 122 & 0.548 & 0.008 & 33.6 & 0.3 & 0.72 \\
\hline 123 & 0.518 & 0.005 & 26.7 & 0.2 & 0.79 \\
\hline 126 & 0.521 & 0.009 & 37.0 & 0.4 & 0.82 \\
\hline 127 & 0.533 & 0.011 & 38.4 & 0.6 & 0.77 \\
\hline 128 & 0.53 & 0.011 & 38.5 & 0.6 & 0.85 \\
\hline 131 & 0.527 & 0.015 & 39.5 & 0.8 & 0.79 \\
\hline 132 & 0.522 & 0.012 & 40.1 & 0.7 & 0.91 \\
\hline 133 & 0.539 & 0.017 & 37.1 & 0.8 & 0.79 \\
\hline 134 & 0.575 & 0.035 & 40.9 & 1.7 & 0.80 \\
\hline 135 & 0.535 & 0.017 & 39.8 & 0.9 & 0.81 \\
\hline 136 & 0.51 & 0.012 & 36.1 & 0.6 & 0.81 \\
\hline 137 & 0.534 & 0.011 & 38.2 & 0.6 & 0.82 \\
\hline 138 & 0.566 & 0.018 & 38.1 & 0.9 & 0.85 \\
\hline 139 & 0.501 & 0.013 & 35.5 & 0.7 & 0.85 \\
\hline 140 & 0.504 & 0.017 & 36.0 & 0.9 & 0.75 \\
\hline 143 & 0.504 & 0.014 & 38.4 & 0.8 & 0.82 \\
\hline 144 & 0.52 & 0.006 & 34.9 & 0.3 & 0.74 \\
\hline 146 & 0.581 & 0.005 & 34.1 & 0.2 & 0.72 \\
\hline
\end{tabular}


$\beta$-chain at $29^{\circ} \mathrm{C}$ and at $14.1 \mathrm{~T}$ in the deoxy form

\begin{tabular}{|c|c|c|c|c|c|c|}
\hline $\begin{array}{c}\text { Residue } \\
\text { No. }\end{array}$ & $\mathbf{R}_{1}$ & $R_{1}$ error & $\mathbf{R}_{\mathbf{2}}$ & $\mathbf{R}_{\mathbf{2}}$ error & NOE & NOE error \\
\hline 3 & 0.532 & 0.034 & 31.3 & 1.4 & 0.57 & 0.05 \\
\hline 4 & 0.419 & 0.011 & 38.0 & 0.7 & 0.74 & 0.05 \\
\hline 6 & 0.41 & 0.005 & 37.6 & 0.3 & 0.66 & 0.05 \\
\hline 7 & 0.416 & 0.008 & 38.5 & 0.5 & 0.74 & 0.05 \\
\hline 8 & 0.416 & 0.009 & 39.3 & 0.6 & 0.77 & 0.05 \\
\hline 9 & 0.413 & 0.005 & 37.5 & 0.4 & 0.75 & 0.05 \\
\hline 10 & 0.427 & 0.007 & 38.6 & 0.5 & 0.81 & 0.05 \\
\hline 11 & 0.405 & 0.006 & 38.1 & 0.4 & 0.82 & 0.05 \\
\hline 12 & 0.433 & 0.007 & 37.0 & 0.4 & 0.71 & 0.05 \\
\hline 13 & 0.426 & 0.007 & 38.8 & 0.5 & 0.81 & 0.05 \\
\hline 15 & 0.409 & 0.012 & 41.2 & 0.9 & 0.82 & 0.05 \\
\hline 16 & 0.41 & 0.007 & 38.5 & 0.5 & 0.83 & 0.05 \\
\hline 17 & 0.4 & 0.007 & 39.5 & 0.5 & 0.78 & 0.05 \\
\hline 19 & 0.433 & 0.006 & 34.3 & 0.3 & 0.73 & 0.05 \\
\hline 20 & 0.524 & 0.004 & 30.2 & 0.1 & 0.45 & 0.05 \\
\hline 21 & 0.543 & 0.005 & 35.6 & 0.2 & 0.64 & 0.05 \\
\hline 23 & 0.419 & 0.008 & 38.3 & 0.5 & 0.76 & 0.05 \\
\hline 24 & 0.453 & 0.009 & 41.0 & 0.6 & 0.79 & 0.05 \\
\hline 25 & 0.434 & 0.008 & 38.4 & 0.5 & 0.69 & 0.05 \\
\hline 26 & 0.421 & 0.008 & 38.1 & 0.5 & 0.82 & 0.05 \\
\hline 27 & 0.425 & 0.01 & 40.3 & 0.7 & 0.85 & 0.05 \\
\hline 29 & 0.39 & 0.009 & 39.5 & 0.7 & 0.82 & 0.05 \\
\hline 30 & 0.416 & 0.009 & 39.7 & 0.6 & 0.84 & 0.05 \\
\hline 31 & 0.394 & 0.023 & 39.5 & 1.6 & 0.72 & 0.05 \\
\hline 33 & 0.411 & 0.028 & 39.6 & 2.1 & 0.88 & 0.05 \\
\hline 34 & 0.421 & 0.023 & 42.7 & 1.7 & 0.90 & 0.05 \\
\hline 35 & 0.4 & 0.012 & 38.8 & 0.9 & 0.92 & 0.05 \\
\hline 38 & 0.433 & 0.014 & 39.5 & 0.9 & 0.84 & 0.05 \\
\hline
\end{tabular}




\begin{tabular}{|c|c|c|c|c|c|c|}
\hline 39 & 0.442 & 0.008 & 39.9 & 0.6 & 0.83 & 0.05 \\
\hline 40 & 0.417 & 0.013 & 38.6 & 1.0 & 0.82 & 0.05 \\
\hline 42 & 0.383 & 0.017 & 36.7 & 1.4 & 0.71 & 0.05 \\
\hline 45 & 0.421 & 0.011 & 38.6 & 0.8 & 0.64 & 0.05 \\
\hline 47 & 0.367 & 0.003 & 32.1 & 0.2 & 0.55 & 0.05 \\
\hline 48 & 0.374 & 0.005 & 31.8 & 0.3 & 0.56 & 0.05 \\
\hline 49 & 0.421 & 0.011 & 34.5 & 0.6 & 0.63 & 0.05 \\
\hline 50 & 0.399 & 0.004 & 33.8 & 0.2 & 0.72 & 0.05 \\
\hline 53 & 0.424 & 0.008 & 37.3 & 0.5 & 0.79 & 0.05 \\
\hline 54 & 0.404 & 0.005 & 37.6 & 0.4 & 0.80 & 0.05 \\
\hline 55 & 0.425 & 0.006 & 37.3 & 0.4 & 0.79 & 0.05 \\
\hline 56 & 0.423 & 0.005 & 35.0 & 0.3 & 0.69 & 0.05 \\
\hline 57 & 0.402 & 0.005 & 36.6 & 0.3 & 0.75 & 0.05 \\
\hline 59 & 0.431 & 0.007 & 39.3 & 0.5 & 0.84 & 0.05 \\
\hline 62 & 0.426 & 0.008 & 38.7 & 0.5 & 0.80 & 0.05 \\
\hline 65 & 0.43 & 0.018 & 37.6 & 1.4 & 0.77 & 0.05 \\
\hline 72 & 0.465 & 0.018 & 37.5 & 1.0 & 0.80 & 0.05 \\
\hline 74 & 0.431 & 0.01 & 37.9 & 0.7 & 0.77 & 0.05 \\
\hline 76 & 0.438 & 0.006 & 38.0 & 0.4 & 0.74 & 0.05 \\
\hline 77 & 0.448 & 0.005 & 34.7 & 0.3 & 0.74 & 0.05 \\
\hline 78 & 0.423 & 0.007 & 39.1 & 0.5 & 0.75 & 0.05 \\
\hline 79 & 0.379 & 0.071 & 36.3 & 3.9 & 0.96 & 0.05 \\
\hline 80 & 0.421 & 0.006 & 37.6 & 0.4 & 0.67 & 0.05 \\
\hline 82 & 0.427 & 0.012 & 39.0 & 0.7 & 0.74 & 0.05 \\
\hline 83 & 0.429 & 0.011 & 36.7 & 0.7 & 0.79 & 0.05 \\
\hline 85 & 0.434 & 0.012 & 37.6 & 0.8 & 0.83 & 0.05 \\
\hline 86 & 0.418 & 0.012 & 37.4 & 0.9 & 0.83 & 0.05 \\
\hline 87 & 0.42 & 0.014 & 36.8 & 1.0 & 0.78 & 0.05 \\
\hline 101 & 0.428 & 0.011 & 39.1 & 0.8 & 0.92 & 0.05 \\
\hline 105 & 0.417 & 0.02 & 39.8 & 1.4 & 0.90 & 0.05 \\
\hline 107 & 0.406 & 0.031 & 43.9 & 2.6 & 0.98 & 0.05 \\
\hline
\end{tabular}




\begin{tabular}{|c|c|c|c|c|c|}
\hline 107 & 0.359 & 0.035 & 28.3 & 1.8 & 0.53 \\
\hline 108 & 0.41 & 0.008 & 41.6 & 0.6 & 0.83 \\
\hline 109 & 0.406 & 0.017 & 51.4 & 1.7 & 0.89 \\
\hline 112 & 0.403 & 0.008 & 41.2 & 0.6 & 0.84 \\
\hline 116 & 0.403 & 0.005 & 40.9 & 0.4 & 0.85 \\
\hline 118 & 0.41 & 0.008 & 39.1 & 0.6 & 0.83 \\
\hline 119 & 0.498 & 0.005 & 34.7 & 0.3 & 0.45 \\
\hline 120 & 0.467 & 0.004 & 34.6 & 0.2 & 0.45 \\
\hline 122 & 0.426 & 0.006 & 36.0 & 0.3 & 0.77 \\
\hline 123 & 0.402 & 0.003 & 30.3 & 0.2 & 0.83 \\
\hline 126 & 0.397 & 0.005 & 38.9 & 0.4 & 0.82 \\
\hline 127 & 0.415 & 0.007 & 42.0 & 0.5 & 0.86 \\
\hline 128 & 0.407 & 0.007 & 40.6 & 0.5 & 0.85 \\
\hline 131 & 0.398 & 0.011 & 41.9 & 0.8 & 0.83 \\
\hline 132 & 0.409 & 0.009 & 44.3 & 0.8 & 0.81 \\
\hline 133 & 0.396 & 0.013 & 41.0 & 1.0 & 0.87 \\
\hline 134 & 0.439 & 0.025 & 42.2 & 1.8 & 0.95 \\
\hline 135 & 0.44 & 0.013 & 40.7 & 0.9 & 0.87 \\
\hline 136 & 0.392 & 0.008 & 38.3 & 0.6 & 0.83 \\
\hline 137 & 0.411 & 0.008 & 40.8 & 0.6 & 0.87 \\
\hline 138 & 0.421 & 0.014 & 40.3 & 1.0 & 0.83 \\
\hline 139 & 0.387 & 0.009 & 36.4 & 0.6 & 0.84 \\
\hline 140 & 0.402 & 0.011 & 37.9 & 0.8 & 0.84 \\
\hline 143 & 0.406 & 0.01 & 41.2 & 0.8 & 0.82 \\
\hline 144 & 0.415 & 0.004 & 36.1 & 0.2 & 0.74 \\
\hline 146 & 0.458 & 0.003 & 35.3 & 0.2 & 0.69 \\
\hline
\end{tabular}


$\beta$-chain at $34{ }^{\circ} \mathrm{C}$ and at $11.7 \mathrm{~T}$ in the deoxy form

\begin{tabular}{|c|c|c|c|c|c|c|}
\hline $\begin{array}{c}\text { Residue } \\
\text { No. }\end{array}$ & $\mathbf{R}_{1}$ & $R_{1}$ error & $\mathbf{R}_{\mathbf{2}}$ & $\mathbf{R}_{\mathbf{2}}$ error & NOE & NOE error \\
\hline 3 & 0.665 & 0.039 & 25.7 & 0.8 & 0.53 & 0.05 \\
\hline 4 & 0.599 & 0.011 & 31.5 & 0.4 & 0.75 & 0.05 \\
\hline 6 & 0.596 & 0.008 & 30.3 & 0.2 & 0.73 & 0.05 \\
\hline 7 & 0.592 & 0.011 & 32.1 & 0.4 & 0.79 & 0.05 \\
\hline 8 & 0.604 & 0.011 & 31.3 & 0.3 & 0.79 & 0.05 \\
\hline 9 & 0.604 & 0.008 & 30.9 & 0.2 & 0.80 & 0.05 \\
\hline 10 & 0.595 & 0.009 & 32.6 & 0.3 & 0.78 & 0.05 \\
\hline 11 & 0.582 & 0.008 & 32.0 & 0.3 & 0.80 & 0.05 \\
\hline 13 & 0.61 & 0.009 & 32.1 & 0.3 & 0.83 & 0.05 \\
\hline 15 & 0.6 & 0.017 & 32.8 & 0.6 & 0.88 & 0.05 \\
\hline 16 & 0.603 & 0.01 & 31.7 & 0.3 & 0.81 & 0.05 \\
\hline 17 & 0.578 & 0.01 & 32.5 & 0.3 & 0.85 & 0.05 \\
\hline 19 & 0.612 & 0.01 & 27.7 & 0.2 & 0.82 & 0.05 \\
\hline 20 & 0.626 & 0.009 & 27.9 & 0.2 & 0.63 & 0.05 \\
\hline 21 & 0.717 & 0.007 & 29.1 & 0.2 & 0.60 & 0.05 \\
\hline 23 & 0.606 & 0.01 & 31.2 & 0.3 & 0.80 & 0.05 \\
\hline 24 & 0.645 & 0.01 & 32.8 & 0.3 & 0.79 & 0.05 \\
\hline 25 & 0.615 & 0.009 & 30.9 & 0.3 & 0.72 & 0.05 \\
\hline 26 & 0.606 & 0.01 & 32.1 & 0.3 & 0.76 & 0.05 \\
\hline 27 & 0.598 & 0.011 & 32.5 & 0.4 & 0.84 & 0.05 \\
\hline 29 & 0.593 & 0.011 & 32.6 & 0.4 & 0.86 & 0.05 \\
\hline 31 & 0.643 & 0.031 & 32.0 & 1.0 & 0.81 & 0.05 \\
\hline 32 & 0.612 & 0.038 & 32.5 & 1.3 & 0.94 & 0.05 \\
\hline 34 & 0.642 & 0.031 & 32.0 & 0.9 & 0.88 & 0.05 \\
\hline 35 & 0.583 & 0.018 & 32.4 & 0.6 & 0.92 & 0.05 \\
\hline 38 & 0.621 & 0.016 & 33.1 & 0.5 & 0.85 & 0.05 \\
\hline 39 & 0.625 & 0.011 & 33.0 & 0.3 & 0.87 & 0.05 \\
\hline 40 & 0.585 & 0.014 & 32.5 & 0.5 & 0.82 & 0.05 \\
\hline
\end{tabular}




\begin{tabular}{|c|c|c|c|c|c|c|}
\hline 42 & 0.572 & 0.019 & 30.7 & 0.6 & 0.79 & 0.05 \\
\hline 45 & 0.597 & 0.011 & 31.7 & 0.3 & 0.68 & 0.05 \\
\hline 47 & 0.512 & 0.005 & 26.2 & 0.2 & 0.64 & 0.05 \\
\hline 48 & 0.539 & 0.007 & 27.0 & 0.2 & 0.69 & 0.05 \\
\hline 49 & 0.592 & 0.015 & 28.2 & 0.4 & 0.71 & 0.05 \\
\hline 50 & 0.566 & 0.005 & 27.8 & 0.2 & 0.76 & 0.05 \\
\hline 53 & 0.592 & 0.01 & 31.2 & 0.3 & 0.84 & 0.05 \\
\hline 54 & 0.593 & 0.008 & 31.1 & 0.2 & 0.81 & 0.05 \\
\hline 55 & 0.602 & 0.009 & 30.2 & 0.3 & 0.81 & 0.05 \\
\hline 56 & 0.603 & 0.008 & 29.6 & 0.2 & 0.72 & 0.05 \\
\hline 57 & 0.555 & 0.007 & 30.8 & 0.2 & 0.79 & 0.05 \\
\hline 59 & 0.614 & 0.007 & 32.5 & 0.2 & 0.85 & 0.05 \\
\hline 60 & 0.603 & 0.013 & 32.7 & 0.4 & 0.83 & 0.05 \\
\hline 62 & 0.612 & 0.008 & 32.4 & 0.3 & 0.83 & 0.05 \\
\hline 65 & 0.573 & 0.016 & 32.1 & 0.5 & 0.79 & 0.05 \\
\hline 72 & 0.632 & 0.017 & 32.2 & 0.5 & 0.89 & 0.05 \\
\hline 74 & 0.617 & 0.012 & 30.4 & 0.3 & 0.81 & 0.05 \\
\hline 77 & 0.622 & 0.008 & 28.7 & 0.2 & 0.71 & 0.05 \\
\hline 78 & 0.59 & 0.012 & 32.2 & 0.4 & 0.74 & 0.05 \\
\hline 80 & 0.614 & 0.009 & 30.2 & 0.3 & 0.69 & 0.05 \\
\hline 82 & 0.601 & 0.017 & 31.7 & 0.5 & 0.76 & 0.05 \\
\hline 83 & 0.604 & 0.014 & 30.3 & 0.4 & 0.77 & 0.05 \\
\hline 85 & 0.608 & 0.014 & 32.0 & 0.5 & 0.79 & 0.05 \\
\hline 86 & 0.602 & 0.014 & 30.6 & 0.4 & 0.85 & 0.05 \\
\hline 87 & 0.586 & 0.02 & 28.6 & 0.6 & 0.80 & 0.05 \\
\hline 101 & 0.626 & 0.013 & 32.3 & 0.4 & 0.80 & 0.05 \\
\hline 105 & 0.596 & 0.019 & 33.2 & 0.6 & 0.86 & 0.05 \\
\hline 106 & 0.555 & 0.029 & 32.3 & 1.0 & 0.87 & 0.05 \\
\hline 107 & 0.602 & 0.023 & 34.3 & 0.8 & 0.90 & 0.05 \\
\hline 108 & 0.584 & 0.009 & 34.4 & 0.3 & 0.80 & 0.05 \\
\hline 109 & 0.59 & 0.016 & 40.9 & 0.7 & 0.85 & 0.05 \\
\hline
\end{tabular}




$\begin{array}{lcccccc}111 & 0.571 & 0.015 & 34.0 & 0.5 & 0.87 & 0.05 \\ 112 & 0.6 & 0.01 & 34.0 & 0.4 & 0.89 & 0.05 \\ 113 & 0.603 & 0.046 & 33.2 & 1.5 & 0.82 & 0.05 \\ 116 & 0.592 & 0.007 & 33.3 & 0.2 & 0.83 & 0.05 \\ 118 & 0.568 & 0.011 & 32.0 & 0.4 & 0.83 & 0.05 \\ 119 & 0.666 & 0.007 & 29.2 & 0.2 & 0.43 & 0.05 \\ 120 & 0.617 & 0.007 & 28.4 & 0.2 & 0.46 & 0.05 \\ 122 & 0.62 & 0.008 & 29.9 & 0.2 & 0.71 & 0.05 \\ 123 & 0.591 & 0.005 & 29.0 & 0.1 & 0.83 & 0.05 \\ 126 & 0.587 & 0.008 & 32.4 & 0.3 & 0.84 & 0.05 \\ 127 & 0.604 & 0.009 & 33.7 & 0.3 & 0.84 & 0.05 \\ 128 & 0.595 & 0.009 & 32.7 & 0.3 & 0.88 & 0.05 \\ 131 & 0.612 & 0.014 & 34.6 & 0.5 & 0.84 & 0.05 \\ 132 & 0.583 & 0.009 & 36.0 & 0.4 & 0.88 & 0.05 \\ 133 & 0.593 & 0.014 & 33.0 & 0.5 & 0.85 & 0.05 \\ 134 & 0.651 & 0.03 & 36.5 & 1.0 & 0.85 & 0.05 \\ 135 & 0.591 & 0.014 & 33.0 & 0.5 & 0.83 & 0.05 \\ 136 & 0.58 & 0.01 & 31.3 & 0.3 & 0.85 & 0.05 \\ 137 & 0.6 & 0.01 & 33.5 & 0.3 & 0.89 & 0.05 \\ 138 & 0.623 & 0.015 & 33.4 & 0.5 & 0.86 & 0.05 \\ 139 & 0.576 & 0.011 & 31.0 & 0.4 & 0.80 & 0.05 \\ 140 & 0.588 & 0.013 & 32.4 & 0.4 & 0.80 & 0.05 \\ 144 & 0.577 & 0.006 & 30.6 & 0.2 & 0.75 & 0.05 \\ 146 & 0.652 & 0.005 & 29.0 & 0.1 & 0.73 & 0.05\end{array}$


$\beta$-chain at $34{ }^{\circ} \mathrm{C}$ and at $14.1 \mathrm{~T}$ in the deoxy form

\begin{tabular}{|c|c|c|c|c|c|c|}
\hline $\begin{array}{c}\text { Residue } \\
\text { No. }\end{array}$ & $\mathbf{R}_{1}$ & $R_{1}$ error & $\mathbf{R}_{\mathbf{2}}$ & $\mathbf{R}_{\mathbf{2}}$ error & NOE & NOE error \\
\hline 3 & 0.592 & 0.04 & 28.1 & 1.1 & 0.41 & 0.05 \\
\hline 4 & 0.475 & 0.009 & 34.5 & 0.4 & 0.70 & 0.05 \\
\hline 6 & 0.452 & 0.005 & 33.2 & 0.2 & 0.73 & 0.05 \\
\hline 7 & 0.464 & 0.007 & 33.4 & 0.3 & 0.73 & 0.05 \\
\hline 8 & 0.47 & 0.008 & 34.4 & 0.4 & 0.79 & 0.05 \\
\hline 9 & 0.465 & 0.005 & 33.2 & 0.2 & 0.74 & 0.05 \\
\hline 10 & 0.465 & 0.005 & 34.0 & 0.3 & 0.78 & 0.05 \\
\hline 11 & 0.449 & 0.005 & 33.5 & 0.3 & 0.80 & 0.05 \\
\hline 13 & 0.463 & 0.006 & 34.2 & 0.3 & 0.82 & 0.05 \\
\hline 15 & 0.463 & 0.01 & 35.6 & 0.6 & 0.83 & 0.05 \\
\hline 16 & 0.456 & 0.006 & 34.0 & 0.3 & 0.84 & 0.05 \\
\hline 17 & 0.446 & 0.006 & 34.8 & 0.3 & 0.78 & 0.05 \\
\hline 19 & 0.473 & 0.007 & 30.0 & 0.3 & 0.73 & 0.05 \\
\hline 20 & 0.504 & 0.006 & 29.1 & 0.2 & 0.62 & 0.05 \\
\hline 21 & 0.584 & 0.005 & 31.5 & 0.2 & 0.61 & 0.05 \\
\hline 23 & 0.472 & 0.007 & 34.1 & 0.3 & 0.76 & 0.05 \\
\hline 24 & 0.513 & 0.007 & 35.9 & 0.3 & 0.75 & 0.05 \\
\hline 25 & 0.479 & 0.006 & 33.0 & 0.3 & 0.74 & 0.05 \\
\hline 26 & 0.459 & 0.006 & 33.2 & 0.3 & 0.79 & 0.05 \\
\hline 27 & 0.457 & 0.008 & 33.9 & 0.4 & 0.79 & 0.05 \\
\hline 29 & 0.442 & 0.008 & 35.0 & 0.4 & 0.83 & 0.05 \\
\hline 31 & 0.451 & 0.02 & 34.8 & 1.0 & 0.84 & 0.05 \\
\hline 32 & 0.433 & 0.026 & 34.7 & 1.5 & 0.86 & 0.05 \\
\hline 35 & 0.427 & 0.011 & 34.0 & 0.6 & 0.91 & 0.05 \\
\hline 38 & 0.496 & 0.012 & 34.5 & 0.5 & 0.87 & 0.05 \\
\hline 39 & 0.479 & 0.007 & 35.2 & 0.4 & 0.83 & 0.05 \\
\hline 40 & 0.472 & 0.01 & 34.1 & 0.6 & 0.80 & 0.05 \\
\hline 42 & 0.445 & 0.014 & 32.5 & 0.8 & 0.86 & 0.05 \\
\hline
\end{tabular}




\begin{tabular}{|c|c|c|c|c|c|c|}
\hline 45 & 0.46 & 0.01 & 33.9 & 0.4 & 0.62 & 0.05 \\
\hline 47 & 0.402 & 0.003 & 28.3 & 0.1 & 0.59 & 0.05 \\
\hline 48 & 0.413 & 0.004 & 28.1 & 0.2 & 0.62 & 0.05 \\
\hline 49 & 0.462 & 0.009 & 30.1 & 0.4 & 0.65 & 0.05 \\
\hline 50 & 0.449 & 0.003 & 29.8 & 0.2 & 0.75 & 0.05 \\
\hline 53 & 0.467 & 0.006 & 32.7 & 0.3 & 0.81 & 0.05 \\
\hline 54 & 0.456 & 0.005 & 32.7 & 0.3 & 0.79 & 0.05 \\
\hline 55 & 0.477 & 0.006 & 32.4 & 0.3 & 0.83 & 0.05 \\
\hline 56 & 0.463 & 0.005 & 31.2 & 0.2 & 0.74 & 0.05 \\
\hline 57 & 0.438 & 0.004 & 32.0 & 0.2 & 0.80 & 0.05 \\
\hline 59 & 0.487 & 0.005 & 34.7 & 0.3 & 0.83 & 0.05 \\
\hline 60 & 0.485 & 0.008 & 34.7 & 0.4 & 0.80 & 0.05 \\
\hline 62 & 0.478 & 0.006 & 34.3 & 0.3 & 0.84 & 0.05 \\
\hline 65 & 0.483 & 0.015 & 33.5 & 0.7 & 0.75 & 0.05 \\
\hline 72 & 0.499 & 0.016 & 34.2 & 0.7 & 0.87 & 0.05 \\
\hline 74 & 0.486 & 0.008 & 33.1 & 0.4 & 0.82 & 0.05 \\
\hline 76 & 0.497 & 0.005 & 33.2 & 0.3 & 0.71 & 0.05 \\
\hline 77 & 0.49 & 0.005 & 30.7 & 0.2 & 0.70 & 0.05 \\
\hline 78 & 0.471 & 0.008 & 34.2 & 0.4 & 0.70 & 0.05 \\
\hline 80 & 0.466 & 0.005 & 32.6 & 0.3 & 0.65 & 0.05 \\
\hline 82 & 0.479 & 0.011 & 34.3 & 0.5 & 0.73 & 0.05 \\
\hline 83 & 0.474 & 0.01 & 32.9 & 0.5 & 0.73 & 0.05 \\
\hline 85 & 0.489 & 0.01 & 33.4 & 0.5 & 0.83 & 0.05 \\
\hline 86 & 0.466 & 0.01 & 33.5 & 0.5 & 0.84 & 0.05 \\
\hline 87 & 0.453 & 0.016 & 32.5 & 0.7 & 0.75 & 0.05 \\
\hline 101 & 0.472 & 0.009 & 34.8 & 0.5 & 0.84 & 0.05 \\
\hline 105 & 0.453 & 0.014 & 35.9 & 0.7 & 0.86 & 0.05 \\
\hline 106 & 0.44 & 0.028 & 34.7 & 1.4 & 0.93 & 0.05 \\
\hline 108 & 0.457 & 0.007 & 37.0 & 0.4 & 0.81 & 0.05 \\
\hline 109 & 0.463 & 0.013 & 44.7 & 0.9 & 0.85 & 0.05 \\
\hline 111 & 0.45 & 0.01 & 38.0 & 0.6 & 0.84 & 0.05 \\
\hline
\end{tabular}




\begin{tabular}{|c|c|c|c|c|c|}
\hline 112 & 0.452 & 0.007 & 36.4 & 0.4 & 0.85 \\
\hline 113 & 0.453 & 0.028 & 38.6 & 1.6 & 0.95 \\
\hline 114 & 0.491 & 0.037 & 38.6 & 2.0 & 0.77 \\
\hline 116 & 0.444 & 0.004 & 36.0 & 0.2 & 0.86 \\
\hline 118 & 0.443 & 0.007 & 34.7 & 0.3 & 0.83 \\
\hline 119 & 0.556 & 0.005 & 30.3 & 0.2 & 0.43 \\
\hline 120 & 0.493 & 0.005 & 30.5 & 0.2 & 0.44 \\
\hline 122 & 0.47 & 0.005 & 31.6 & 0.2 & 0.72 \\
\hline 123 & 0.448 & 0.003 & 30.7 & 0.1 & 0.83 \\
\hline 126 & 0.449 & 0.004 & 34.1 & 0.2 & 0.79 \\
\hline 127 & 0.468 & 0.006 & 37.3 & 0.3 & 0.85 \\
\hline 128 & 0.458 & 0.006 & 35.9 & 0.3 & 0.84 \\
\hline 131 & 0.45 & 0.009 & 38.2 & 0.5 & 0.82 \\
\hline 132 & 0.447 & 0.007 & 38.9 & 0.4 & 0.87 \\
\hline 133 & 0.439 & 0.01 & 36.2 & 0.6 & 0.80 \\
\hline 134 & 0.481 & 0.02 & 40.8 & 1.2 & 0.88 \\
\hline 135 & 0.481 & 0.011 & 35.0 & 0.6 & 0.91 \\
\hline 136 & 0.444 & 0.007 & 33.7 & 0.3 & 0.84 \\
\hline 137 & 0.472 & 0.007 & 35.7 & 0.4 & 0.87 \\
\hline 138 & 0.475 & 0.01 & 36.9 & 0.6 & 0.88 \\
\hline 139 & 0.441 & 0.008 & 31.9 & 0.4 & 0.83 \\
\hline 140 & 0.442 & 0.009 & 34.9 & 0.5 & 0.84 \\
\hline 143 & 0.459 & 0.008 & 37.2 & 0.5 & 0.80 \\
\hline 144 & 0.457 & 0.004 & 32.0 & 0.2 & 0.72 \\
\hline 146 & 0.521 & 0.003 & 30.5 & 0.1 & 0.68 \\
\hline
\end{tabular}


Table 2S. NMR Relaxation Parameters of Backbone Amide ${ }^{15} \mathrm{~N}$ in $\mathrm{HbCO}$ A Errors in relaxation rates were estimated by Monte Carlo procedure when fitting the exponential decays, while errors for the NOE were estimated based on the signal-to-noise of the spectra as reported by NMRPipe.

Units: $\mathrm{R}_{1}, \mathrm{~s}^{-1} ; \mathrm{R}_{2}, \mathrm{~s}^{-1}$; NOE, none

$\alpha$-chain at $29^{\circ} \mathrm{C}$ and at $11.7 \mathrm{~T}$ in the $\mathrm{CO}$ form

\begin{tabular}{|c|c|c|c|c|c|c|}
\hline $\begin{array}{c}\text { Residue } \\
\text { No. }\end{array}$ & $\mathbf{R}_{1}$ & $R_{1}$ error & $\mathbf{R}_{\mathbf{2}}$ & $\mathbf{R}_{\mathbf{2}}$ error & NOE & NOE error \\
\hline 3 & 0.613 & 0.014 & 35.0 & 0.6 & 0.65 & 0.05 \\
\hline 6 & 0.518 & 0.013 & 37.3 & 0.6 & 0.76 & 0.05 \\
\hline 7 & 0.545 & 0.019 & 38.1 & 1.0 & 0.63 & 0.05 \\
\hline 8 & 0.527 & 0.015 & 35.9 & 0.8 & 0.84 & 0.05 \\
\hline 9 & 0.513 & 0.016 & 38.7 & 1.1 & 0.64 & 0.05 \\
\hline 10 & 0.515 & 0.011 & 37.4 & 0.6 & 0.94 & 0.05 \\
\hline 11 & 0.52 & 0.015 & 37.0 & 0.8 & 0.73 & 0.05 \\
\hline 12 & 0.513 & 0.015 & 37.7 & 0.6 & 0.82 & 0.05 \\
\hline 13 & 0.511 & 0.017 & 37.8 & 0.4 & 0.78 & 0.05 \\
\hline 14 & 0.54 & 0.014 & 38.0 & 0.8 & 0.81 & 0.05 \\
\hline 15 & 0.536 & 0.014 & 36.9 & 0.7 & 0.80 & 0.05 \\
\hline 16 & 0.509 & 0.007 & 36.6 & 0.4 & 0.70 & 0.05 \\
\hline 17 & 0.543 & 0.009 & 35.4 & 0.5 & 0.66 & 0.05 \\
\hline 18 & 0.547 & 0.007 & 32.9 & 0.3 & 0.56 & 0.05 \\
\hline 20 & 0.622 & 0.01 & 30.8 & 0.4 & 0.40 & 0.05 \\
\hline 21 & 0.557 & 0.01 & 36.0 & 0.5 & 0.81 & 0.05 \\
\hline 23 & 0.544 & 0.008 & 34.3 & 0.4 & 0.73 & 0.05 \\
\hline 24 & 0.535 & 0.014 & 36.6 & 0.7 & 0.83 & 0.05 \\
\hline 25 & 0.53 & 0.011 & 35.6 & 0.5 & 0.80 & 0.05 \\
\hline 26 & 0.536 & 0.015 & 38.0 & 0.9 & 0.88 & 0.05 \\
\hline 28 & 0.558 & 0.018 & 37.4 & 0.5 & 0.84 & 0.05 \\
\hline 29 & 0.521 & 0.016 & 34.4 & 0.8 & 0.91 & 0.05 \\
\hline 30 & 0.521 & 0.012 & 38.7 & 0.6 & 0.67 & 0.05 \\
\hline
\end{tabular}




\begin{tabular}{|c|c|c|c|c|c|c|}
\hline 31 & 0.533 & 0.014 & 34.9 & 0.7 & 0.84 & 0.05 \\
\hline 32 & 0.519 & 0.012 & 37.5 & 0.7 & 0.85 & 0.05 \\
\hline 33 & 0.542 & 0.018 & 36.7 & 0.8 & 0.87 & 0.05 \\
\hline 34 & 0.603 & 0.015 & 30.6 & 0.5 & 0.66 & 0.05 \\
\hline 35 & 0.524 & 0.019 & 33.0 & 0.9 & 0.94 & 0.05 \\
\hline 36 & 0.514 & 0.011 & 36.4 & 0.6 & 0.80 & 0.05 \\
\hline 38 & 0.543 & 0.03 & 37.0 & 1.6 & 0.87 & 0.05 \\
\hline 39 & 0.545 & 0.035 & 40.7 & 1.9 & 0.83 & 0.05 \\
\hline 40 & 0.555 & 0.011 & 39.6 & 0.5 & 0.81 & 0.05 \\
\hline 41 & 0.582 & 0.027 & 35.5 & 1.3 & 0.80 & 0.05 \\
\hline 42 & 0.545 & 0.02 & 36.2 & 1.0 & 0.85 & 0.05 \\
\hline 45 & 0.528 & 0.012 & 33.4 & 0.6 & 0.75 & 0.05 \\
\hline 46 & 0.493 & 0.01 & 35.4 & 0.5 & 0.78 & 0.05 \\
\hline 47 & 0.529 & 0.006 & 34.9 & 0.3 & 0.71 & 0.05 \\
\hline 48 & 0.528 & 0.007 & 31.0 & 0.3 & 0.75 & 0.05 \\
\hline 51 & 0.532 & 0.02 & 30.9 & 0.8 & 0.46 & 0.05 \\
\hline 52 & 0.537 & 0.008 & 32.3 & 0.3 & 0.73 & 0.05 \\
\hline 55 & 0.531 & 0.013 & 36.4 & 0.6 & 0.81 & 0.05 \\
\hline 57 & 0.546 & 0.01 & 35.1 & 0.5 & 0.80 & 0.05 \\
\hline 58 & 0.554 & 0.011 & 36.8 & 0.6 & 0.83 & 0.05 \\
\hline 59 & 0.545 & 0.013 & 37.0 & 0.7 & 0.86 & 0.05 \\
\hline 60 & 0.567 & 0.011 & 36.9 & 0.6 & 0.81 & 0.05 \\
\hline 61 & 0.525 & 0.012 & 35.4 & 0.5 & 0.77 & 0.05 \\
\hline 63 & 0.518 & 0.007 & 37.4 & 0.3 & 0.79 & 0.05 \\
\hline 65 & 0.55 & 0.012 & 36.8 & 0.6 & 0.79 & 0.05 \\
\hline 67 & 0.526 & 0.016 & 34.9 & 0.7 & 0.78 & 0.05 \\
\hline 68 & 0.58 & 0.015 & 34.5 & 1.1 & 0.84 & 0.05 \\
\hline 69 & 0.534 & 0.011 & 36.0 & 0.6 & 0.80 & 0.05 \\
\hline 70 & 0.519 & 0.013 & 35.3 & 0.6 & 0.83 & 0.05 \\
\hline 72 & 0.522 & 0.009 & 32.9 & 0.4 & 0.70 & 0.05 \\
\hline 73 & 0.519 & 0.01 & 35.3 & 0.5 & 0.59 & 0.05 \\
\hline
\end{tabular}




\begin{tabular}{|c|c|c|c|c|c|c|}
\hline 74 & 0.544 & 0.009 & 35.9 & 0.4 & 0.65 & 0.05 \\
\hline 75 & 0.516 & 0.01 & 35.6 & 0.5 & 0.63 & 0.05 \\
\hline 76 & 0.567 & 0.01 & 32.8 & 0.4 & 0.58 & 0.05 \\
\hline 78 & 0.539 & 0.012 & 32.1 & 0.5 & 0.84 & 0.05 \\
\hline 79 & 0.517 & 0.007 & 36.4 & 0.4 & 0.67 & 0.05 \\
\hline 80 & 0.512 & 0.014 & 34.0 & 0.7 & 0.79 & 0.05 \\
\hline 83 & 0.537 & 0.012 & 33.5 & 0.6 & 0.73 & 0.05 \\
\hline 86 & 0.743 & 0.05 & 34.1 & 1.7 & 0.51 & 0.05 \\
\hline 87 & 0.538 & 0.036 & 35.5 & 1.7 & 0.84 & 0.05 \\
\hline 89 & 0.53 & 0.014 & 33.3 & 0.6 & 0.68 & 0.05 \\
\hline 90 & 0.531 & 0.019 & 36.2 & 1.0 & 0.72 & 0.05 \\
\hline 92 & 0.542 & 0.019 & 35.0 & 0.8 & 0.74 & 0.05 \\
\hline 93 & 0.548 & 0.027 & 34.0 & 1.2 & 0.69 & 0.05 \\
\hline 94 & 0.587 & 0.033 & 42.5 & 1.9 & 0.78 & 0.05 \\
\hline 100 & 0.517 & 0.013 & 37.7 & 0.7 & 0.78 & 0.05 \\
\hline 102 & 0.504 & 0.019 & 37.0 & 1.1 & 0.83 & 0.05 \\
\hline 103 & 0.526 & 0.01 & 38.0 & 0.5 & 0.93 & 0.05 \\
\hline 105 & 0.489 & 0.02 & 38.7 & 1.2 & 0.80 & 0.05 \\
\hline 107 & 0.539 & 0.009 & 35.6 & 0.4 & 0.76 & 0.05 \\
\hline 109 & 0.525 & 0.01 & 37.0 & 0.6 & 0.66 & 0.05 \\
\hline 111 & 0.511 & 0.009 & 34.8 & 0.4 & 0.81 & 0.05 \\
\hline 112 & 0.482 & 0.01 & 35.2 & 0.5 & 0.82 & 0.05 \\
\hline 116 & 0.603 & 0.015 & 30.6 & 0.5 & 0.66 & 0.05 \\
\hline 117 & 0.501 & 0.012 & 36.1 & 0.7 & 0.80 & 0.05 \\
\hline 122 & 0.527 & 0.011 & 37.6 & 0.6 & 0.84 & 0.05 \\
\hline 123 & 0.509 & 0.017 & 38.2 & 0.9 & 0.76 & 0.05 \\
\hline 124 & 0.524 & 0.012 & 35.5 & 0.6 & 0.71 & 0.05 \\
\hline 125 & 0.528 & 0.01 & 38.4 & 0.6 & 0.77 & 0.05 \\
\hline 126 & 0.526 & 0.013 & 38.1 & 0.7 & 0.83 & 0.05 \\
\hline 127 & 0.53 & 0.019 & 39.1 & 1.0 & 0.87 & 0.05 \\
\hline 128 & 0.507 & 0.011 & 38.4 & 0.6 & 0.81 & 0.05 \\
\hline
\end{tabular}




$\begin{array}{lllllll}129 & 0.527 & 0.019 & 38.4 & 1.1 & 0.84 & 0.05 \\ 130 & 0.528 & 0.016 & 37.3 & 0.9 & 0.75 & 0.05 \\ 131 & 0.512 & 0.014 & 37.2 & 0.5 & 0.70 & 0.05 \\ 132 & 0.509 & 0.016 & 38.8 & 0.8 & 0.82 & 0.05 \\ 133 & 0.508 & 0.025 & 36.9 & 1.4 & 0.62 & 0.05 \\ 134 & 0.529 & 0.016 & 37.8 & 0.8 & 0.61 & 0.05 \\ 135 & 0.54 & 0.016 & 38.2 & 0.8 & 0.84 & 0.05\end{array}$


$\alpha$-chain at $29{ }^{\circ} \mathrm{C}$ and at $14.1 \mathrm{~T}$ in the CO form

\begin{tabular}{|c|c|c|c|c|c|c|}
\hline $\begin{array}{c}\text { Residue } \\
\text { No. }\end{array}$ & $\mathbf{R}_{1}$ & $R_{1}$ error & $\mathbf{R}_{\mathbf{2}}$ & $\mathbf{R}_{\mathbf{2}}$ error & NOE & NOE error \\
\hline 3 & 0.511 & 0.015 & 36.7 & 0.7 & 0.68 & 0.05 \\
\hline 6 & 0.413 & 0.014 & 39.6 & 0.9 & 0.79 & 0.05 \\
\hline 7 & 0.427 & 0.019 & 40.6 & 1.3 & 0.74 & 0.05 \\
\hline 8 & 0.407 & 0.013 & 38.8 & 0.9 & 0.76 & 0.05 \\
\hline 9 & 0.421 & 0.019 & 40.4 & 1.1 & 0.86 & 0.05 \\
\hline 10 & 0.412 & 0.01 & 41.5 & 0.7 & 0.90 & 0.05 \\
\hline 11 & 0.426 & 0.015 & 41.0 & 1.0 & 0.83 & 0.05 \\
\hline 12 & 0.413 & 0.013 & 43.8 & 0.9 & 0.82 & 0.05 \\
\hline 13 & 0.413 & 0.009 & 39.9 & 0.5 & 0.81 & 0.05 \\
\hline 14 & 0.417 & 0.013 & 38.4 & 0.9 & 0.82 & 0.05 \\
\hline 15 & 0.44 & 0.013 & 40.1 & 0.9 & 0.82 & 0.05 \\
\hline 16 & 0.407 & 0.007 & 39.8 & 0.5 & 0.72 & 0.05 \\
\hline 17 & 0.437 & 0.009 & 36.9 & 0.5 & 0.68 & 0.05 \\
\hline 18 & 0.456 & 0.006 & 33.3 & 0.3 & 0.57 & 0.05 \\
\hline 20 & 0.488 & 0.01 & 31.2 & 0.4 & 0.36 & 0.05 \\
\hline 21 & 0.443 & 0.009 & 39.0 & 0.5 & 0.76 & 0.05 \\
\hline 22 & 0.42 & 0.014 & 37.0 & 0.9 & 0.78 & 0.05 \\
\hline 23 & 0.427 & 0.007 & 37.0 & 0.4 & 0.70 & 0.05 \\
\hline 24 & 0.418 & 0.013 & 39.5 & 0.9 & 0.72 & 0.05 \\
\hline 25 & 0.423 & 0.01 & 37.7 & 0.6 & 0.86 & 0.05 \\
\hline 26 & 0.417 & 0.011 & 41.7 & 0.7 & 0.81 & 0.05 \\
\hline 28 & 0.427 & 0.01 & 40.7 & 0.7 & 0.87 & 0.05 \\
\hline 29 & 0.399 & 0.016 & 36.4 & 1.0 & 0.82 & 0.05 \\
\hline 30 & 0.418 & 0.012 & 40.5 & 0.8 & 0.70 & 0.05 \\
\hline 31 & 0.417 & 0.012 & 36.6 & 0.7 & 0.87 & 0.05 \\
\hline 32 & 0.417 & 0.011 & 40.7 & 0.7 & 0.81 & 0.05 \\
\hline 33 & 0.414 & 0.016 & 39.4 & 1.0 & 0.77 & 0.05 \\
\hline 34 & 0.51 & 0.015 & 32.4 & 0.6 & 0.73 & 0.05 \\
\hline
\end{tabular}




\begin{tabular}{|c|c|c|c|c|c|c|}
\hline 35 & 0.429 & 0.016 & 33.3 & 0.9 & 0.77 & 0.05 \\
\hline 36 & 0.406 & 0.009 & 38.2 & 0.8 & 0.85 & 0.05 \\
\hline 38 & 0.478 & 0.033 & 37.3 & 1.8 & 0.76 & 0.05 \\
\hline 39 & 0.418 & 0.032 & 41.2 & 2.1 & 0.80 & 0.05 \\
\hline 40 & 0.433 & 0.01 & 42.9 & 0.8 & 0.78 & 0.05 \\
\hline 41 & 0.444 & 0.026 & 36.6 & 1.4 & 0.73 & 0.05 \\
\hline 42 & 0.441 & 0.018 & 39.0 & 1.2 & 0.71 & 0.05 \\
\hline 43 & 0.403 & 0.01 & 35.5 & 0.6 & 0.84 & 0.05 \\
\hline 45 & 0.432 & 0.011 & 35.3 & 0.7 & 0.77 & 0.05 \\
\hline 46 & 0.399 & 0.008 & 39.7 & 0.6 & 0.82 & 0.05 \\
\hline 47 & 0.419 & 0.005 & 36.5 & 0.3 & 0.59 & 0.05 \\
\hline 48 & 0.413 & 0.006 & 32.7 & 0.3 & 0.73 & 0.05 \\
\hline 49 & 0.463 & 0.011 & 30.1 & 0.9 & 0.68 & 0.05 \\
\hline 51 & 0.412 & 0.02 & 31.8 & 0.9 & 0.47 & 0.05 \\
\hline 52 & 0.454 & 0.007 & 33.9 & 0.3 & 0.71 & 0.05 \\
\hline 55 & 0.432 & 0.011 & 39.1 & 0.7 & 0.80 & 0.05 \\
\hline 56 & 0.427 & 0.006 & 39.8 & 0.4 & 0.85 & 0.05 \\
\hline 57 & 0.442 & 0.008 & 38.1 & 0.5 & 0.87 & 0.05 \\
\hline 58 & 0.446 & 0.01 & 40.1 & 0.7 & 0.85 & 0.05 \\
\hline 59 & 0.412 & 0.01 & 39.7 & 0.7 & 0.81 & 0.05 \\
\hline 60 & 0.44 & 0.01 & 40.6 & 0.7 & 0.79 & 0.05 \\
\hline 61 & 0.422 & 0.01 & 37.5 & 0.7 & 0.82 & 0.05 \\
\hline 63 & 0.417 & 0.006 & 40.3 & 0.4 & 0.82 & 0.05 \\
\hline 65 & 0.443 & 0.01 & 39.6 & 0.7 & 0.92 & 0.05 \\
\hline 66 & 0.417 & 0.011 & 39.2 & 0.7 & 0.87 & 0.05 \\
\hline 67 & 0.404 & 0.013 & 37.8 & 0.8 & 0.83 & 0.05 \\
\hline 68 & 0.42 & 0.006 & 40.5 & 0.5 & 0.85 & 0.05 \\
\hline 69 & 0.434 & 0.011 & 38.5 & 0.7 & 0.83 & 0.05 \\
\hline 70 & 0.412 & 0.012 & 34.6 & 0.7 & 0.83 & 0.05 \\
\hline 72 & 0.409 & 0.008 & 34.2 & 0.5 & 0.73 & 0.05 \\
\hline 73 & 0.426 & 0.011 & 39.2 & 0.7 & 0.70 & 0.05 \\
\hline
\end{tabular}




\begin{tabular}{|c|c|c|c|c|c|c|}
\hline 74 & 0.436 & 0.008 & 38.8 & 0.5 & 0.70 & 0.05 \\
\hline 75 & 0.428 & 0.011 & 38.3 & 0.6 & 0.58 & 0.05 \\
\hline 76 & 0.452 & 0.009 & 36.3 & 0.5 & 0.51 & 0.05 \\
\hline 78 & 0.412 & 0.009 & 31.9 & 0.5 & 0.67 & 0.05 \\
\hline 79 & 0.419 & 0.007 & 39.6 & 0.5 & 0.60 & 0.05 \\
\hline 80 & 0.396 & 0.013 & 32.6 & 0.8 & 0.75 & 0.05 \\
\hline 83 & 0.431 & 0.01 & 36.0 & 0.7 & 0.77 & 0.05 \\
\hline 84 & 0.425 & 0.013 & 40.2 & 0.8 & 0.90 & 0.05 \\
\hline 86 & 0.624 & 0.071 & 34.2 & 2.8 & 0.58 & 0.05 \\
\hline 87 & 0.449 & 0.036 & 36.1 & 2.2 & 0.73 & 0.05 \\
\hline 89 & 0.412 & 0.014 & 33.4 & 0.7 & 0.66 & 0.05 \\
\hline 90 & 0.439 & 0.022 & 39.6 & 1.5 & 0.69 & 0.05 \\
\hline 92 & 0.416 & 0.019 & 37.3 & 1.2 & 0.78 & 0.05 \\
\hline 94 & 0.416 & 0.034 & 48.8 & 3.0 & 0.73 & 0.05 \\
\hline 97 & 0.439 & 0.038 & 45.9 & 2.5 & 0.87 & 0.05 \\
\hline 100 & 0.407 & 0.015 & 39.8 & 1.1 & 0.80 & 0.05 \\
\hline 103 & 0.416 & 0.011 & 41.9 & 0.8 & 0.88 & 0.05 \\
\hline 105 & 0.388 & 0.018 & 43.0 & 1.5 & 0.81 & 0.05 \\
\hline 107 & 0.375 & 0.012 & 38.0 & 0.6 & 0.81 & 0.05 \\
\hline 108 & 0.401 & 0.019 & 37.4 & 1.3 & 0.82 & 0.05 \\
\hline 109 & 0.413 & 0.01 & 43.3 & 1.0 & 0.77 & 0.05 \\
\hline 110 & 0.419 & 0.007 & 39.7 & 0.4 & 0.78 & 0.05 \\
\hline 111 & 0.414 & 0.008 & 36.4 & 0.5 & 0.83 & 0.05 \\
\hline 112 & 0.388 & 0.009 & 36.6 & 0.6 & 0.78 & 0.05 \\
\hline 115 & 0.428 & 0.015 & 37.3 & 0.8 & 0.66 & 0.05 \\
\hline 117 & 0.403 & 0.013 & 40.4 & 0.9 & 0.74 & 0.05 \\
\hline 120 & 0.408 & 0.007 & 37.3 & 0.5 & 0.86 & 0.05 \\
\hline 122 & 0.42 & 0.01 & 40.9 & 0.7 & 0.93 & 0.05 \\
\hline 123 & 0.396 & 0.016 & 43.1 & 1.4 & 0.92 & 0.05 \\
\hline 124 & 0.405 & 0.012 & 37.5 & 0.7 & 0.77 & 0.05 \\
\hline 125 & 0.413 & 0.01 & 41.1 & 0.7 & 0.83 & 0.05 \\
\hline
\end{tabular}




$\begin{array}{lllllll}126 & 0.422 & 0.014 & 39.7 & 0.8 & 0.77 & 0.05 \\ 127 & 0.407 & 0.02 & 40.7 & 1.6 & 0.84 & 0.05 \\ 128 & 0.41 & 0.01 & 41.2 & 0.7 & 0.81 & 0.05 \\ 129 & 0.416 & 0.018 & 41.5 & 1.3 & 0.89 & 0.05 \\ 130 & 0.42 & 0.018 & 41.1 & 1.3 & 0.70 & 0.05 \\ 131 & 0.408 & 0.012 & 40.2 & 0.8 & 0.79 & 0.05 \\ 132 & 0.403 & 0.013 & 41.9 & 1.0 & 0.85 & 0.05 \\ 133 & 0.444 & 0.024 & 35.7 & 1.2 & 0.86 & 0.05 \\ 134 & 0.425 & 0.016 & 40.8 & 1.1 & 0.88 & 0.05\end{array}$


$\alpha$-chain at $34^{\circ} \mathrm{C}$ and at $11.7 \mathrm{~T}$ in the CO form

\begin{tabular}{|c|c|c|c|c|c|c|}
\hline $\begin{array}{c}\text { Residue } \\
\text { No. }\end{array}$ & $\mathbf{R}_{1}$ & $R_{1}$ error & $\mathbf{R}_{\mathbf{2}}$ & $\mathbf{R}_{\mathbf{2}}$ error & NOE & NOE error \\
\hline 3 & 0.687 & 0.016 & 29.2 & 0.4 & 0.65 & 0.05 \\
\hline 6 & 0.639 & 0.025 & 32.5 & 0.5 & 0.75 & 0.05 \\
\hline 8 & 0.627 & 0.013 & 32.7 & 0.5 & 0.81 & 0.05 \\
\hline 10 & 0.601 & 0.01 & 31.9 & 0.4 & 0.74 & 0.05 \\
\hline 11 & 0.621 & 0.016 & 32.7 & 0.7 & 0.92 & 0.05 \\
\hline 12 & 0.604 & 0.012 & 32.7 & 0.4 & 0.84 & 0.05 \\
\hline 13 & 0.579 & 0.009 & 33.1 & 0.3 & 0.88 & 0.05 \\
\hline 14 & 0.604 & 0.012 & 33.0 & 0.5 & 0.82 & 0.05 \\
\hline 16 & 0.591 & 0.007 & 31.5 & 0.3 & 0.73 & 0.05 \\
\hline 17 & 0.609 & 0.007 & 30.3 & 0.2 & 0.65 & 0.05 \\
\hline 18 & 0.631 & 0.007 & 28.5 & 0.2 & 0.52 & 0.05 \\
\hline 20 & 0.671 & 0.01 & 26.1 & 0.3 & 0.28 & 0.05 \\
\hline 21 & 0.621 & 0.008 & 31.6 & 0.3 & 0.75 & 0.05 \\
\hline 22 & 0.594 & 0.028 & 32.0 & 1.1 & 0.73 & 0.05 \\
\hline 23 & 0.619 & 0.008 & 30.5 & 0.3 & 0.69 & 0.05 \\
\hline 24 & 0.629 & 0.012 & 31.3 & 0.4 & 0.84 & 0.05 \\
\hline 25 & 0.615 & 0.01 & 31.3 & 0.4 & 0.80 & 0.05 \\
\hline 26 & 0.613 & 0.012 & 33.0 & 0.4 & 0.83 & 0.05 \\
\hline 28 & 0.623 & 0.012 & 32.0 & 0.4 & 0.96 & 0.05 \\
\hline 29 & 0.6 & 0.017 & 30.3 & 0.6 & 0.84 & 0.05 \\
\hline 30 & 0.598 & 0.013 & 33.5 & 0.5 & 0.80 & 0.05 \\
\hline 31 & 0.623 & 0.014 & 30.3 & 0.5 & 0.83 & 0.05 \\
\hline 32 & 0.613 & 0.012 & 33.2 & 0.5 & 0.84 & 0.05 \\
\hline 33 & 0.604 & 0.015 & 32.6 & 0.5 & 0.80 & 0.05 \\
\hline 34 & 0.698 & 0.016 & 27.0 & 0.4 & 0.76 & 0.05 \\
\hline 35 & 0.596 & 0.019 & 28.5 & 0.6 & 0.78 & 0.05 \\
\hline 36 & 0.596 & 0.01 & 31.1 & 0.4 & 0.78 & 0.05 \\
\hline 38 & 0.588 & 0.048 & 32.7 & 1.9 & 0.76 & 0.05 \\
\hline
\end{tabular}




\begin{tabular}{|c|c|c|c|c|c|c|}
\hline 39 & 0.616 & 0.033 & 33.5 & 1.3 & 0.85 & 0.05 \\
\hline 40 & 0.633 & 0.013 & 34.6 & 0.5 & 0.79 & 0.05 \\
\hline 41 & 0.654 & 0.028 & 32.2 & 1.0 & 0.79 & 0.05 \\
\hline 42 & 0.615 & 0.019 & 31.2 & 0.7 & 0.77 & 0.05 \\
\hline 43 & 0.61 & 0.011 & 29.3 & 0.4 & 0.81 & 0.05 \\
\hline 45 & 0.598 & 0.012 & 29.5 & 0.4 & 0.76 & 0.05 \\
\hline 46 & 0.587 & 0.008 & 32.1 & 0.3 & 0.75 & 0.05 \\
\hline 48 & 0.583 & 0.007 & 27.5 & 0.2 & 0.72 & 0.05 \\
\hline 49 & 0.614 & 0.011 & 27.4 & 0.5 & 0.75 & 0.05 \\
\hline 51 & 0.615 & 0.035 & 27.5 & 1.1 & 0.44 & 0.05 \\
\hline 52 & 0.618 & 0.007 & 27.8 & 0.2 & 0.63 & 0.05 \\
\hline 55 & 0.614 & 0.011 & 31.4 & 0.4 & 0.79 & 0.05 \\
\hline 57 & 0.638 & 0.009 & 31.3 & 0.4 & 0.78 & 0.05 \\
\hline 58 & 0.614 & 0.01 & 32.4 & 0.4 & 0.81 & 0.05 \\
\hline 59 & 0.609 & 0.011 & 32.4 & 0.4 & 0.77 & 0.05 \\
\hline 60 & 0.626 & 0.01 & 32.1 & 0.4 & 0.81 & 0.05 \\
\hline 61 & 0.606 & 0.011 & 30.6 & 0.4 & 0.76 & 0.05 \\
\hline 63 & 0.612 & 0.006 & 32.8 & 0.2 & 0.83 & 0.05 \\
\hline 65 & 0.599 & 0.011 & 32.0 & 0.4 & 0.79 & 0.05 \\
\hline 66 & 0.594 & 0.01 & 31.5 & 0.4 & 0.75 & 0.05 \\
\hline 67 & 0.6 & 0.013 & 31.4 & 0.5 & 0.81 & 0.05 \\
\hline 69 & 0.626 & 0.012 & 31.9 & 0.4 & 0.83 & 0.05 \\
\hline 70 & 0.613 & 0.013 & 30.3 & 0.5 & 0.94 & 0.05 \\
\hline 71 & 0.622 & 0.006 & 31.3 & 0.2 & 0.80 & 0.05 \\
\hline 72 & 0.593 & 0.008 & 27.9 & 0.3 & 0.75 & 0.05 \\
\hline 73 & 0.593 & 0.009 & 30.4 & 0.4 & 0.70 & 0.05 \\
\hline 74 & 0.606 & 0.008 & 30.6 & 0.3 & 0.75 & 0.05 \\
\hline 75 & 0.59 & 0.01 & 31.4 & 0.4 & 0.61 & 0.05 \\
\hline 76 & 0.624 & 0.011 & 29.2 & 0.3 & 0.56 & 0.05 \\
\hline 78 & 0.578 & 0.01 & 27.0 & 0.4 & 0.65 & 0.05 \\
\hline 79 & 0.599 & 0.008 & 31.3 & 0.3 & 0.66 & 0.05 \\
\hline
\end{tabular}




\begin{tabular}{|c|c|c|c|c|c|c|}
\hline 80 & 0.584 & 0.016 & 28.1 & 0.5 & 0.80 & 0.05 \\
\hline 83 & 0.621 & 0.013 & 29.4 & 0.4 & 0.77 & 0.05 \\
\hline 84 & 0.649 & 0.021 & 31.3 & 0.7 & 0.87 & 0.05 \\
\hline 86 & 0.825 & 0.041 & 26.8 & 0.9 & 0.57 & 0.05 \\
\hline 87 & 0.609 & 0.033 & 31.7 & 1.3 & 0.74 & 0.05 \\
\hline 89 & 0.572 & 0.012 & 29.1 & 0.4 & 0.74 & 0.05 \\
\hline 90 & 0.589 & 0.019 & 30.2 & 0.7 & 0.78 & 0.05 \\
\hline 92 & 0.606 & 0.017 & 29.3 & 0.7 & 0.75 & 0.05 \\
\hline 93 & 0.613 & 0.022 & 29.4 & 0.9 & 0.74 & 0.05 \\
\hline 94 & 0.62 & 0.029 & 35.1 & 1.2 & 0.88 & 0.05 \\
\hline 100 & 0.585 & 0.013 & 32.4 & 0.6 & 0.82 & 0.05 \\
\hline 102 & 0.557 & 0.018 & 32.4 & 0.8 & 0.83 & 0.05 \\
\hline 103 & 0.599 & 0.012 & 32.5 & 0.5 & 0.79 & 0.05 \\
\hline 105 & 0.593 & 0.02 & 34.1 & 0.9 & 0.75 & 0.05 \\
\hline 107 & 0.578 & 0.014 & 33.0 & 0.5 & 0.75 & 0.05 \\
\hline 109 & 0.602 & 0.011 & 32.4 & 0.4 & 0.82 & 0.05 \\
\hline 110 & 0.599 & 0.008 & 31.7 & 0.3 & 0.83 & 0.05 \\
\hline 111 & 0.592 & 0.009 & 29.3 & 0.3 & 0.81 & 0.05 \\
\hline 112 & 0.541 & 0.009 & 30.5 & 0.4 & 0.76 & 0.05 \\
\hline 115 & 0.589 & 0.012 & 30.8 & 0.5 & 0.79 & 0.05 \\
\hline 117 & 0.585 & 0.012 & 32.4 & 0.5 & 0.80 & 0.05 \\
\hline 120 & 0.59 & 0.006 & 31.7 & 0.3 & 0.80 & 0.05 \\
\hline 122 & 0.602 & 0.011 & 33.0 & 0.4 & 0.80 & 0.05 \\
\hline 124 & 0.578 & 0.012 & 32.3 & 0.4 & 0.79 & 0.05 \\
\hline 125 & 0.605 & 0.01 & 33.7 & 0.4 & 0.82 & 0.05 \\
\hline 126 & 0.627 & 0.012 & 33.0 & 0.5 & 0.83 & 0.05 \\
\hline 127 & 0.588 & 0.016 & 33.3 & 0.6 & 0.79 & 0.05 \\
\hline 128 & 0.586 & 0.011 & 34.4 & 0.5 & 0.80 & 0.05 \\
\hline 129 & 0.62 & 0.018 & 32.9 & 0.7 & 0.78 & 0.05 \\
\hline 130 & 0.615 & 0.017 & 33.3 & 0.7 & 0.78 & 0.05 \\
\hline 131 & 0.567 & 0.012 & 32.1 & 0.6 & 0.95 & 0.05 \\
\hline
\end{tabular}




$\begin{array}{lllllll}132 & 0.601 & 0.015 & 33.5 & 0.7 & 1.01 & 0.05 \\ 133 & 0.584 & 0.023 & 30.9 & 0.8 & 0.76 & 0.05 \\ 134 & 0.595 & 0.015 & 32.9 & 0.7 & 0.80 & 0.05\end{array}$


$\alpha$-chain at $34{ }^{\circ} \mathrm{C}$ and at $14.1 \mathrm{~T}$ in the $\mathrm{CO}$ form

\begin{tabular}{|c|c|c|c|c|c|c|}
\hline $\begin{array}{c}\text { Residue } \\
\text { No. }\end{array}$ & $\mathbf{R}_{1}$ & $\mathbf{R}_{\mathbf{1}}$ error & $\mathbf{R}_{\mathbf{2}}$ & $\mathbf{R}_{\mathbf{2}}$ error & NOE & NOE erro \\
\hline 3 & 0.532 & 0.012 & 32.0 & 0.6 & 0.70 & 0.05 \\
\hline 6 & 0.458 & 0.012 & 35.8 & 0.7 & 0.69 & 0.05 \\
\hline 7 & 0.466 & 0.013 & 35.7 & 0.7 & 0.87 & 0.05 \\
\hline 8 & 0.461 & 0.011 & 33.2 & 0.6 & 0.78 & 0.05 \\
\hline 9 & 0.45 & 0.014 & 35.7 & 0.7 & 0.81 & 0.05 \\
\hline 10 & 0.467 & 0.008 & 36.7 & 0.5 & 0.85 & 0.05 \\
\hline 11 & 0.458 & 0.013 & 37.3 & 0.8 & 0.78 & 0.05 \\
\hline 12 & 0.479 & 0.01 & 38.2 & 0.6 & 0.82 & 0.05 \\
\hline 13 & 0.432 & 0.008 & 35.9 & 0.5 & 0.74 & 0.05 \\
\hline 14 & 0.463 & 0.011 & 34.5 & 0.6 & 0.83 & 0.05 \\
\hline 15 & 0.487 & 0.01 & 35.2 & 0.6 & 0.77 & 0.05 \\
\hline 16 & 0.454 & 0.006 & 35.7 & 0.4 & 0.74 & 0.05 \\
\hline 17 & 0.471 & 0.005 & 33.8 & 0.3 & 0.70 & 0.05 \\
\hline 18 & 0.497 & 0.005 & 30.3 & 0.2 & 0.61 & 0.05 \\
\hline 20 & 0.54 & 0.008 & 27.4 & 0.3 & 0.40 & 0.05 \\
\hline 21 & 0.48 & 0.007 & 34.3 & 0.4 & 0.78 & 0.05 \\
\hline 22 & 0.451 & 0.023 & 33.4 & 1.1 & 0.76 & 0.05 \\
\hline 23 & 0.486 & 0.005 & 32.4 & 0.3 & 0.72 & 0.05 \\
\hline 24 & 0.495 & 0.011 & 36.5 & 0.7 & 0.75 & 0.05 \\
\hline 25 & 0.468 & 0.008 & 35.6 & 0.5 & 0.87 & 0.05 \\
\hline 26 & 0.484 & 0.008 & 38.4 & 0.5 & 0.82 & 0.05 \\
\hline 28 & 0.474 & 0.008 & 36.3 & 0.5 & 0.84 & 0.05 \\
\hline 29 & 0.457 & 0.013 & 31.9 & 0.6 & 0.93 & 0.05 \\
\hline 31 & 0.459 & 0.01 & 32.5 & 0.5 & 0.84 & 0.05 \\
\hline 32 & 0.481 & 0.009 & 36.3 & 0.5 & 0.80 & 0.05 \\
\hline 33 & 0.474 & 0.01 & 34.6 & 0.6 & 0.93 & 0.05 \\
\hline 35 & 0.465 & 0.013 & 30.3 & 0.7 & 0.87 & 0.05 \\
\hline 36 & 0.461 & 0.007 & 33.4 & 0.4 & 0.84 & 0.05 \\
\hline
\end{tabular}




\begin{tabular}{|c|c|c|c|c|c|c|}
\hline 38 & 0.48 & 0.049 & 31.2 & 2.2 & 0.77 & 0.05 \\
\hline 39 & 0.477 & 0.025 & 34.4 & 1.4 & 0.77 & 0.05 \\
\hline 40 & 0.5 & 0.01 & 38.4 & 0.5 & 0.92 & 0.05 \\
\hline 41 & 0.506 & 0.022 & 32.2 & 1.1 & 0.85 & 0.05 \\
\hline 42 & 0.493 & 0.015 & 33.0 & 0.8 & 0.86 & 0.05 \\
\hline 43 & 0.453 & 0.008 & 31.9 & 0.4 & 0.84 & 0.05 \\
\hline 45 & 0.457 & 0.009 & 31.4 & 0.5 & 0.76 & 0.05 \\
\hline 46 & 0.463 & 0.007 & 34.0 & 0.4 & 0.76 & 0.05 \\
\hline 47 & 0.468 & 0.004 & 33.3 & 0.2 & 0.78 & 0.05 \\
\hline 48 & 0.46 & 0.005 & 28.4 & 0.2 & 0.69 & 0.05 \\
\hline 49 & 0.484 & 0.007 & 32.5 & 0.4 & 0.58 & 0.05 \\
\hline 51 & 0.468 & 0.029 & 30.2 & 1.2 & 0.53 & 0.05 \\
\hline 52 & 0.483 & 0.006 & 30.3 & 0.3 & 0.67 & 0.05 \\
\hline 54 & 0.473 & 0.009 & 35.6 & 0.5 & 0.79 & 0.05 \\
\hline 55 & 0.477 & 0.01 & 35.9 & 0.5 & 0.78 & 0.05 \\
\hline 57 & 0.5 & 0.007 & 34.5 & 0.4 & 0.78 & 0.05 \\
\hline 58 & 0.503 & 0.008 & 34.2 & 0.4 & 0.86 & 0.05 \\
\hline 59 & 0.469 & 0.008 & 35.7 & 0.5 & 0.85 & 0.05 \\
\hline 60 & 0.493 & 0.008 & 36.1 & 0.5 & 0.94 & 0.05 \\
\hline 61 & 0.482 & 0.009 & 33.8 & 0.5 & 0.86 & 0.05 \\
\hline 63 & 0.462 & 0.005 & 36.8 & 0.3 & 0.83 & 0.05 \\
\hline 65 & 0.476 & 0.009 & 33.6 & 0.4 & 0.83 & 0.05 \\
\hline 66 & 0.465 & 0.008 & 35.1 & 0.4 & 0.81 & 0.05 \\
\hline 67 & 0.457 & 0.01 & 34.1 & 0.6 & 0.83 & 0.05 \\
\hline 68 & 0.486 & 0.008 & 36.8 & 0.4 & 0.83 & 0.05 \\
\hline 69 & 0.469 & 0.009 & 34.3 & 0.5 & 0.85 & 0.05 \\
\hline 70 & 0.457 & 0.01 & 32.0 & 0.6 & 0.88 & 0.05 \\
\hline 72 & 0.463 & 0.007 & 29.7 & 0.3 & 0.81 & 0.05 \\
\hline 73 & 0.482 & 0.009 & 34.3 & 0.5 & 0.63 & 0.05 \\
\hline 74 & 0.48 & 0.008 & 34.8 & 0.4 & 0.61 & 0.05 \\
\hline 75 & 0.464 & 0.009 & 35.0 & 0.5 & 0.59 & 0.05 \\
\hline
\end{tabular}




\begin{tabular}{|c|c|c|c|c|c|c|}
\hline 76 & 0.502 & 0.01 & 31.4 & 0.4 & 0.58 & 0.05 \\
\hline 78 & 0.456 & 0.009 & 28.4 & 0.4 & 0.74 & 0.05 \\
\hline 79 & 0.472 & 0.007 & 35.4 & 0.4 & 0.67 & 0.05 \\
\hline 80 & 0.46 & 0.012 & 30.0 & 0.6 & 0.76 & 0.05 \\
\hline 83 & 0.488 & 0.01 & 33.1 & 0.5 & 0.68 & 0.05 \\
\hline 84 & 0.495 & 0.009 & 36.0 & 0.5 & 0.71 & 0.05 \\
\hline 86 & 0.667 & 0.043 & 29.7 & 1.3 & 0.51 & 0.05 \\
\hline 87 & 0.491 & 0.028 & 33.2 & 1.6 & 0.82 & 0.05 \\
\hline 89 & 0.46 & 0.01 & 29.4 & 0.5 & 0.83 & 0.05 \\
\hline 90 & 0.479 & 0.017 & 33.6 & 1.0 & 0.81 & 0.05 \\
\hline 92 & 0.489 & 0.017 & 32.2 & 0.9 & 0.75 & 0.05 \\
\hline 93 & 0.521 & 0.026 & 34.7 & 1.2 & 0.83 & 0.05 \\
\hline 94 & 0.484 & 0.026 & 44.7 & 1.7 & 0.97 & 0.05 \\
\hline 98 & 0.462 & 0.013 & 36.4 & 0.8 & 0.86 & 0.05 \\
\hline 100 & 0.456 & 0.014 & 35.6 & 0.8 & 0.80 & 0.05 \\
\hline 102 & 0.427 & 0.013 & 34.3 & 0.9 & 0.84 & 0.05 \\
\hline 103 & 0.458 & 0.01 & 36.9 & 0.5 & 0.84 & 0.05 \\
\hline 105 & 0.437 & 0.014 & 36.5 & 0.9 & 0.81 & 0.05 \\
\hline 107 & 0.47 & 0.007 & 34.2 & 0.4 & 0.88 & 0.05 \\
\hline 108 & 0.454 & 0.016 & 33.9 & 0.9 & 0.78 & 0.05 \\
\hline 110 & 0.461 & 0.008 & 36.7 & 0.5 & 0.81 & 0.05 \\
\hline 111 & 0.454 & 0.006 & 32.9 & 0.4 & 0.85 & 0.05 \\
\hline 112 & 0.44 & 0.007 & 32.0 & 0.4 & 0.93 & 0.05 \\
\hline 115 & 0.459 & 0.014 & 33.6 & 0.7 & 0.78 & 0.05 \\
\hline 116 & 0.526 & 0.01 & 28.4 & 0.5 & 0.74 & 0.05 \\
\hline 117 & 0.456 & 0.009 & 34.9 & 0.5 & 0.76 & 0.05 \\
\hline 120 & 0.458 & 0.006 & 33.7 & 0.3 & 0.83 & 0.05 \\
\hline 121 & 0.465 & 0.01 & 36.0 & 0.4 & 0.98 & 0.05 \\
\hline 122 & 0.469 & 0.009 & 36.5 & 0.5 & 0.89 & 0.05 \\
\hline 124 & 0.445 & 0.009 & 31.9 & 0.5 & 0.79 & 0.05 \\
\hline 125 & 0.471 & 0.008 & 36.9 & 0.5 & 0.84 & 0.05 \\
\hline
\end{tabular}




$\begin{array}{lllllll}126 & 0.457 & 0.01 & 35.7 & 0.7 & 0.87 & 0.05 \\ 127 & 0.459 & 0.017 & 34.5 & 1.0 & 0.78 & 0.05 \\ 128 & 0.455 & 0.008 & 37.4 & 0.6 & 0.89 & 0.05 \\ 129 & 0.469 & 0.015 & 37.6 & 0.9 & 0.84 & 0.05 \\ 130 & 0.466 & 0.016 & 35.4 & 0.9 & 0.83 & 0.05 \\ 131 & 0.453 & 0.01 & 35.0 & 0.6 & 0.87 & 0.05 \\ 132 & 0.47 & 0.011 & 36.9 & 0.6 & 0.80 & 0.05 \\ 133 & 0.44 & 0.017 & 31.3 & 1.0 & 0.81 & 0.05 \\ 135 & 0.46 & 0.014 & 38.1 & 0.9 & 0.83 & 0.05\end{array}$


$\beta$-chain at $29^{\circ} \mathrm{C}$ and at $11.7 \mathrm{~T}$ in the CO form

\begin{tabular}{|c|c|c|c|c|c|c|}
\hline $\begin{array}{c}\text { Residue } \\
\text { No. }\end{array}$ & $\mathbf{R}_{\mathbf{1}}$ & $\mathbf{R}_{\mathbf{1}}$ error & $\mathbf{R}_{\mathbf{2}}$ & $\mathbf{R}_{\mathbf{2}}$ error & NOE & NOE erro \\
\hline 4 & 0.527 & 0.005 & 32.9 & 0.3 & 0.67 & 0.05 \\
\hline 6 & 0.508 & 0.007 & 33.6 & 0.3 & 0.75 & 0.05 \\
\hline 7 & 0.528 & 0.012 & 35.9 & 0.6 & 0.71 & 0.05 \\
\hline 8 & 0.528 & 0.008 & 35.5 & 0.4 & 0.77 & 0.05 \\
\hline 9 & 0.53 & 0.007 & 34.0 & 0.4 & 0.72 & 0.05 \\
\hline 10 & 0.534 & 0.007 & 36.5 & 0.4 & 0.73 & 0.05 \\
\hline 12 & 0.521 & 0.008 & 35.6 & 0.4 & 0.80 & 0.05 \\
\hline 16 & 0.531 & 0.009 & 35.1 & 0.5 & 0.82 & 0.05 \\
\hline 18 & 0.517 & 0.008 & 35.8 & 0.4 & 0.85 & 0.05 \\
\hline 19 & 0.535 & 0.007 & 33.3 & 0.3 & 0.75 & 0.05 \\
\hline 20 & 0.617 & 0.007 & 33.3 & 0.3 & 0.56 & 0.05 \\
\hline 21 & 0.666 & 0.007 & 34.0 & 0.2 & 0.64 & 0.05 \\
\hline 24 & 0.553 & 0.012 & 37.1 & 0.7 & 0.75 & 0.05 \\
\hline 25 & 0.529 & 0.01 & 34.5 & 0.5 & 0.78 & 0.05 \\
\hline 26 & 0.531 & 0.009 & 37.7 & 0.5 & 0.77 & 0.05 \\
\hline 29 & 0.504 & 0.01 & 38.5 & 0.6 & 0.82 & 0.05 \\
\hline 31 & 0.507 & 0.015 & 38.2 & 0.9 & 0.85 & 0.05 \\
\hline 32 & 0.509 & 0.024 & 35.7 & 1.4 & 0.86 & 0.05 \\
\hline 33 & 0.502 & 0.022 & 35.2 & 1.3 & 0.82 & 0.05 \\
\hline 34 & 0.545 & 0.027 & 35.1 & 1.3 & 0.80 & 0.05 \\
\hline 35 & 0.521 & 0.02 & 35.3 & 1.1 & 0.82 & 0.05 \\
\hline 37 & 0.551 & 0.032 & 42.7 & 2.3 & 0.84 & 0.05 \\
\hline 38 & 0.541 & 0.024 & 37.5 & 1.1 & 0.79 & 0.05 \\
\hline 41 & 0.543 & 0.016 & 28.4 & 0.7 & 0.78 & 0.05 \\
\hline 42 & 0.507 & 0.013 & 34.1 & 0.7 & 0.75 & 0.05 \\
\hline 43 & 0.536 & 0.008 & 34.3 & 0.4 & 0.35 & 0.05 \\
\hline 45 & 0.528 & 0.009 & 35.2 & 0.5 & 0.67 & 0.05 \\
\hline 46 & 0.498 & 0.005 & 33.3 & 0.2 & 0.50 & 0.05 \\
\hline
\end{tabular}




\begin{tabular}{|c|c|c|c|c|c|c|}
\hline 47 & 0.481 & 0.004 & 30.2 & 0.2 & 0.52 & 0.05 \\
\hline 48 & 0.494 & 0.006 & 32.6 & 0.3 & 0.54 & 0.05 \\
\hline 49 & 0.551 & 0.015 & 32.2 & 0.6 & 0.60 & 0.05 \\
\hline 50 & 0.478 & 0.005 & 34.4 & 0.3 & 0.66 & 0.05 \\
\hline 52 & 0.513 & 0.006 & 32.4 & 0.3 & 0.71 & 0.05 \\
\hline 53 & 0.538 & 0.01 & 35.8 & 0.6 & 0.78 & 0.05 \\
\hline 54 & 0.523 & 0.006 & 34.9 & 0.3 & 0.80 & 0.05 \\
\hline 55 & 0.535 & 0.009 & 34.5 & 0.5 & 0.78 & 0.05 \\
\hline 56 & 0.524 & 0.008 & 34.3 & 0.4 & 0.71 & 0.05 \\
\hline 57 & 0.5 & 0.005 & 35.5 & 0.3 & 0.75 & 0.05 \\
\hline 60 & 0.502 & 0.014 & 34.2 & 0.7 & 0.76 & 0.05 \\
\hline 62 & 0.534 & 0.005 & 36.4 & 0.3 & 0.75 & 0.05 \\
\hline 63 & 0.519 & 0.012 & 36.9 & 0.5 & 0.90 & 0.05 \\
\hline 65 & 0.553 & 0.009 & 37.5 & 0.5 & 0.80 & 0.05 \\
\hline 66 & 0.528 & 0.01 & 35.7 & 0.6 & 0.81 & 0.05 \\
\hline 67 & 0.522 & 0.013 & 37.1 & 0.7 & 0.80 & 0.05 \\
\hline 68 & 0.536 & 0.011 & 36.9 & 0.5 & 0.78 & 0.05 \\
\hline 69 & 0.536 & 0.01 & 35.4 & 0.5 & 0.81 & 0.05 \\
\hline 70 & 0.536 & 0.012 & 37.9 & 0.7 & 0.76 & 0.05 \\
\hline 71 & 0.518 & 0.012 & 35.3 & 0.6 & 0.81 & 0.05 \\
\hline 72 & 0.507 & 0.011 & 34.7 & 0.7 & 0.74 & 0.05 \\
\hline 73 & 0.532 & 0.01 & 36.9 & 0.6 & 0.79 & 0.05 \\
\hline 79 & 0.542 & 0.01 & 34.5 & 0.5 & 0.66 & 0.05 \\
\hline 81 & 0.575 & 0.039 & 30.9 & 1.5 & 0.60 & 0.05 \\
\hline 82 & 0.53 & 0.013 & 34.7 & 0.7 & 0.69 & 0.05 \\
\hline 83 & 0.565 & 0.011 & 33.4 & 0.5 & 0.70 & 0.05 \\
\hline 84 & 0.562 & 0.013 & 34.0 & 0.7 & 0.87 & 0.05 \\
\hline 85 & 0.55 & 0.01 & 33.1 & 0.5 & 0.75 & 0.05 \\
\hline 86 & 0.524 & 0.009 & 36.2 & 0.4 & 0.78 & 0.05 \\
\hline 88 & 0.553 & 0.012 & 37.0 & 0.6 & 0.81 & 0.05 \\
\hline 89 & 0.546 & 0.013 & 37.9 & 0.7 & 0.83 & 0.05 \\
\hline
\end{tabular}




\begin{tabular}{|c|c|c|c|c|c|c|}
\hline 90 & 0.525 & 0.014 & 37.8 & 0.8 & 0.79 & 0.05 \\
\hline 92 & 0.506 & 0.014 & 36.5 & 0.8 & 0.80 & 0.05 \\
\hline 93 & 0.516 & 0.02 & 34.5 & 1.1 & 0.87 & 0.05 \\
\hline 94 & 0.477 & 0.016 & 34.2 & 0.8 & 0.72 & 0.05 \\
\hline 95 & 0.498 & 0.012 & 34.9 & 0.7 & 0.82 & 0.05 \\
\hline 96 & 0.522 & 0.015 & 32.3 & 0.7 & 0.73 & 0.05 \\
\hline 97 & 0.532 & 0.013 & 33.6 & 0.6 & 0.73 & 0.05 \\
\hline 99 & 0.569 & 0.014 & 35.6 & 0.6 & 0.79 & 0.05 \\
\hline 101 & 0.547 & 0.022 & 39.7 & 1.3 & 0.82 & 0.05 \\
\hline 102 & 0.512 & 0.016 & 37.0 & 1.0 & 0.77 & 0.05 \\
\hline 104 & 0.512 & 0.017 & 37.4 & 0.8 & 0.75 & 0.05 \\
\hline 106 & 0.512 & 0.013 & 38.7 & 0.8 & 0.77 & 0.05 \\
\hline 108 & 0.522 & 0.011 & 39.7 & 0.5 & 0.82 & 0.05 \\
\hline 109 & 0.507 & 0.01 & 40.1 & 0.6 & 0.84 & 0.05 \\
\hline 110 & 0.457 & 0.031 & 38.0 & 2.0 & 0.84 & 0.05 \\
\hline 112 & 0.485 & 0.011 & 37.5 & 0.7 & 0.82 & 0.05 \\
\hline 113 & 0.509 & 0.033 & 38.3 & 1.5 & 0.85 & 0.05 \\
\hline 115 & 0.493 & 0.015 & 38.7 & 0.9 & 0.69 & 0.05 \\
\hline 116 & 0.498 & 0.008 & 37.5 & 0.5 & 0.85 & 0.05 \\
\hline 117 & 0.509 & 0.009 & 37.7 & 0.5 & 0.81 & 0.05 \\
\hline 118 & 0.49 & 0.009 & 35.6 & 0.5 & 0.78 & 0.05 \\
\hline 119 & 0.558 & 0.007 & 34.0 & 0.3 & 0.43 & 0.05 \\
\hline 120 & 0.546 & 0.006 & 33.6 & 0.3 & 0.44 & 0.05 \\
\hline 121 & 0.536 & 0.006 & 34.2 & 0.3 & 0.71 & 0.05 \\
\hline 122 & 0.533 & 0.007 & 35.2 & 0.4 & 0.72 & 0.05 \\
\hline 123 & 0.506 & 0.005 & 34.3 & 0.2 & 0.81 & 0.05 \\
\hline 126 & 0.507 & 0.007 & 37.7 & 0.5 & 0.86 & 0.05 \\
\hline 128 & 0.521 & 0.014 & 38.8 & 0.6 & 0.87 & 0.05 \\
\hline 129 & 0.501 & 0.007 & 38.0 & 0.4 & 0.82 & 0.05 \\
\hline 130 & 0.498 & 0.014 & 37.8 & 0.8 & 0.89 & 0.05 \\
\hline 131 & 0.5 & 0.012 & 39.1 & 0.7 & 0.82 & 0.05 \\
\hline
\end{tabular}




$\begin{array}{lllllll}132 & 0.506 & 0.013 & 39.3 & 0.7 & 0.82 & 0.05 \\ 133 & 0.507 & 0.019 & 36.8 & 1.1 & 0.75 & 0.05 \\ 134 & 0.501 & 0.025 & 40.0 & 1.6 & 0.90 & 0.05 \\ 135 & 0.55 & 0.013 & 40.0 & 0.8 & 0.96 & 0.05 \\ 136 & 0.477 & 0.013 & 38.9 & 0.9 & 0.83 & 0.05 \\ 137 & 0.513 & 0.014 & 38.5 & 0.8 & 0.82 & 0.05 \\ 138 & 0.529 & 0.013 & 38.3 & 0.8 & 0.89 & 0.05 \\ 139 & 0.506 & 0.011 & 36.6 & 0.6 & 0.78 & 0.05 \\ 140 & 0.528 & 0.013 & 38.4 & 0.7 & 0.73 & 0.05 \\ 143 & 0.553 & 0.021 & 37.8 & 1.1 & 0.76 & 0.05 \\ 144 & 0.581 & 0.027 & 40.0 & 1.4 & 0.79 & 0.05 \\ 146 & 0.772 & 0.011 & 28.5 & 0.3 & 0.53 & 0.05\end{array}$


$\beta$-chain at $29^{\circ} \mathrm{C}$ and at $14.1 \mathrm{~T}$ in the CO form

\begin{tabular}{|c|c|c|c|c|c|c|}
\hline $\begin{array}{c}\text { Residue } \\
\text { No. }\end{array}$ & $\mathbf{R}_{\mathbf{1}}$ & $\mathbf{R}_{\mathbf{1}}$ error & $\mathbf{R}_{\mathbf{2}}$ & $\mathbf{R}_{\mathbf{2}}$ error & NOE & NOE erro \\
\hline 4 & 0.433 & 0.005 & 34.4 & 0.3 & 0.71 & 0.05 \\
\hline 6 & 0.415 & 0.014 & 42.2 & 1.0 & 0.76 & 0.05 \\
\hline 7 & 0.42 & 0.007 & 38.7 & 0.6 & 0.72 & 0.05 \\
\hline 8 & 0.429 & 0.006 & 36.8 & 0.5 & 0.73 & 0.05 \\
\hline 9 & 0.438 & 0.006 & 35.9 & 0.4 & 0.71 & 0.05 \\
\hline 10 & 0.424 & 0.005 & 39.2 & 0.4 & 0.71 & 0.05 \\
\hline 12 & 0.422 & 0.006 & 37.4 & 0.5 & 0.64 & 0.05 \\
\hline 16 & 0.428 & 0.007 & 37.6 & 0.5 & 0.68 & 0.05 \\
\hline 18 & 0.416 & 0.006 & 37.8 & 0.5 & 0.75 & 0.05 \\
\hline 20 & 0.517 & 0.005 & 35.4 & 0.3 & 0.63 & 0.05 \\
\hline 21 & 0.576 & 0.006 & 35.8 & 0.3 & 0.61 & 0.05 \\
\hline 24 & 0.448 & 0.009 & 41.3 & 0.7 & 0.69 & 0.05 \\
\hline 26 & 0.428 & 0.007 & 41.2 & 0.5 & 0.76 & 0.05 \\
\hline 28 & 0.417 & 0.007 & 38.2 & 0.6 & 0.78 & 0.05 \\
\hline 29 & 0.412 & 0.008 & 40.0 & 0.7 & 0.82 & 0.05 \\
\hline 34 & 0.442 & 0.025 & 38.7 & 1.7 & 0.84 & 0.05 \\
\hline 35 & 0.402 & 0.018 & 37.2 & 1.3 & 0.81 & 0.05 \\
\hline 37 & 0.418 & 0.031 & 46.5 & 3.4 & 0.81 & 0.05 \\
\hline 38 & 0.418 & 0.02 & 40.4 & 1.3 & 0.85 & 0.05 \\
\hline 41 & 0.433 & 0.013 & 27.0 & 0.8 & 0.75 & 0.05 \\
\hline 42 & 0.408 & 0.01 & 34.2 & 0.7 & 0.75 & 0.05 \\
\hline 46 & 0.413 & 0.004 & 36.3 & 0.3 & 0.41 & 0.05 \\
\hline 47 & 0.401 & 0.004 & 32.9 & 0.2 & 0.46 & 0.05 \\
\hline 48 & 0.396 & 0.005 & 32.8 & 0.3 & 0.44 & 0.05 \\
\hline 52 & 0.419 & 0.004 & 35.3 & 0.3 & 0.66 & 0.05 \\
\hline 53 & 0.442 & 0.007 & 39.3 & 0.6 & 0.70 & 0.05 \\
\hline 56 & 0.43 & 0.006 & 36.0 & 0.4 & 0.62 & 0.05 \\
\hline 57 & 0.409 & 0.005 & 38.5 & 0.4 & 0.75 & 0.05 \\
\hline
\end{tabular}




\begin{tabular}{|c|c|c|c|c|c|c|}
\hline 60 & 0.402 & 0.01 & 37.2 & 0.8 & 0.72 & 0.05 \\
\hline 62 & 0.44 & 0.006 & 38.1 & 0.4 & 0.72 & 0.05 \\
\hline 65 & 0.449 & 0.007 & 39.8 & 0.6 & 0.79 & 0.05 \\
\hline 66 & 0.419 & 0.007 & 37.5 & 0.6 & 0.79 & 0.05 \\
\hline 67 & 0.417 & 0.01 & 38.5 & 0.7 & 0.78 & 0.05 \\
\hline 69 & 0.438 & 0.008 & 38.3 & 0.6 & 0.75 & 0.05 \\
\hline 70 & 0.436 & 0.008 & 39.6 & 0.6 & 0.79 & 0.05 \\
\hline 71 & 0.421 & 0.009 & 36.9 & 0.7 & 0.77 & 0.05 \\
\hline 72 & 0.414 & 0.009 & 36.6 & 0.6 & 0.79 & 0.05 \\
\hline 73 & 0.417 & 0.008 & 39.8 & 0.6 & 0.75 & 0.05 \\
\hline 74 & 0.431 & 0.008 & 38.8 & 0.6 & 0.77 & 0.05 \\
\hline 79 & 0.456 & 0.009 & 37.2 & 0.5 & 0.58 & 0.05 \\
\hline 82 & 0.463 & 0.012 & 37.3 & 0.8 & 0.70 & 0.05 \\
\hline 83 & 0.479 & 0.009 & 35.6 & 0.6 & 0.59 & 0.05 \\
\hline 85 & 0.439 & 0.008 & 34.9 & 0.5 & 0.74 & 0.05 \\
\hline 86 & 0.428 & 0.006 & 38.9 & 0.5 & 0.76 & 0.05 \\
\hline 88 & 0.453 & 0.01 & 39.8 & 0.8 & 0.75 & 0.05 \\
\hline 89 & 0.437 & 0.011 & 40.7 & 0.8 & 0.80 & 0.05 \\
\hline 90 & 0.42 & 0.009 & 40.0 & 0.8 & 0.79 & 0.05 \\
\hline 92 & 0.434 & 0.013 & 38.6 & 0.9 & 0.87 & 0.05 \\
\hline 93 & 0.44 & 0.018 & 36.1 & 1.2 & 0.75 & 0.05 \\
\hline 94 & 0.395 & 0.014 & 34.5 & 1.1 & 0.71 & 0.05 \\
\hline 95 & 0.407 & 0.009 & 34.9 & 0.7 & 0.71 & 0.05 \\
\hline 96 & 0.416 & 0.011 & 35.5 & 0.9 & 0.74 & 0.05 \\
\hline 97 & 0.446 & 0.009 & 36.3 & 0.9 & 0.83 & 0.05 \\
\hline 99 & 0.46 & 0.014 & 38.9 & 0.9 & 0.70 & 0.05 \\
\hline 101 & 0.428 & 0.022 & 45.3 & 2.2 & 0.74 & 0.05 \\
\hline 102 & 0.421 & 0.014 & 39.9 & 1.1 & 0.76 & 0.05 \\
\hline 104 & 0.427 & 0.013 & 40.4 & 1.1 & 0.63 & 0.05 \\
\hline 106 & 0.41 & 0.012 & 40.7 & 0.9 & 0.79 & 0.05 \\
\hline 109 & 0.412 & 0.009 & 44.3 & 0.8 & 0.80 & 0.05 \\
\hline
\end{tabular}




$\begin{array}{lllllll}112 & 0.397 & 0.009 & 40.6 & 0.7 & 0.80 & 0.05 \\ 116 & 0.401 & 0.006 & 39.2 & 0.5 & 0.79 & 0.05 \\ 117 & 0.406 & 0.007 & 38.8 & 0.6 & 0.79 & 0.05 \\ 118 & 0.397 & 0.007 & 38.0 & 0.6 & 0.75 & 0.05 \\ 119 & 0.474 & 0.006 & 36.6 & 0.4 & 0.42 & 0.05 \\ 120 & 0.453 & 0.005 & 35.1 & 0.3 & 0.44 & 0.05 \\ 122 & 0.434 & 0.005 & 37.5 & 0.4 & 0.69 & 0.05 \\ 123 & 0.5 & 0.024 & 43.6 & 1.9 & 0.88 & 0.05 \\ 127 & 0.432 & 0.009 & 38.4 & 0.6 & 0.76 & 0.05 \\ 128 & 0.42 & 0.009 & 41.6 & 0.7 & 0.81 & 0.05 \\ 129 & 0.398 & 0.005 & 41.1 & 0.5 & 0.78 & 0.05 \\ 130 & 0.398 & 0.011 & 39.2 & 0.9 & 0.89 & 0.05 \\ 131 & 0.395 & 0.01 & 40.0 & 0.8 & 0.83 & 0.05 \\ 132 & 0.41 & 0.01 & 41.3 & 0.9 & 0.79 & 0.05 \\ 133 & 0.399 & 0.013 & 40.2 & 1.1 & 0.79 & 0.05 \\ 135 & 0.425 & 0.011 & 42.1 & 0.9 & 0.77 & 0.05 \\ 136 & 0.41 & 0.012 & 40.2 & 1.0 & 0.77 & 0.05 \\ 137 & 0.426 & 0.006 & 35.7 & 0.3 & 0.77 & 0.05 \\ 138 & 0.426 & 0.011 & 40.8 & 0.9 & 0.80 & 0.05 \\ 139 & 0.413 & 0.01 & 37.7 & 0.8 & 0.76 & 0.05 \\ 143 & 0.463 & 0.017 & 41.6 & 1.3 & 0.75 & 0.05 \\ 146 & 0.669 & 0.01 & 30.1 & 0.3 & 0.53 & 0.05\end{array}$


$\beta$-chain at $34{ }^{\circ} \mathrm{C}$ and at $11.7 \mathrm{~T}$ in the CO form

\begin{tabular}{|c|c|c|c|c|c|c|}
\hline $\begin{array}{c}\text { Residue } \\
\text { No. }\end{array}$ & $\mathbf{R}_{\mathbf{1}}$ & $\mathbf{R}_{\mathbf{1}}$ error & $\mathbf{R}_{\mathbf{2}}$ & $\mathbf{R}_{\mathbf{2}}$ error & NOE & NOE erro \\
\hline 3 & 0.677 & 0.007 & 23.4 & 0.2 & 0.28 & 0.05 \\
\hline 4 & 0.601 & 0.007 & 28.7 & 0.2 & 0.69 & 0.05 \\
\hline 6 & 0.575 & 0.008 & 30.7 & 0.3 & 0.72 & 0.05 \\
\hline 7 & 0.605 & 0.012 & 32.1 & 0.5 & 0.69 & 0.05 \\
\hline 8 & 0.617 & 0.01 & 31.8 & 0.4 & 0.76 & 0.05 \\
\hline 9 & 0.601 & 0.009 & 31.6 & 0.3 & 0.73 & 0.05 \\
\hline 10 & 0.609 & 0.009 & 32.2 & 0.3 & 0.75 & 0.05 \\
\hline 12 & 0.596 & 0.008 & 31.2 & 0.3 & 0.81 & 0.05 \\
\hline 13 & 0.617 & 0.011 & 31.5 & 0.4 & 0.81 & 0.05 \\
\hline 16 & 0.602 & 0.011 & 31.7 & 0.4 & 0.79 & 0.05 \\
\hline 18 & 0.585 & 0.008 & 31.5 & 0.3 & 0.76 & 0.05 \\
\hline 19 & 0.608 & 0.009 & 28.2 & 0.3 & 0.68 & 0.05 \\
\hline 20 & 0.694 & 0.009 & 28.4 & 0.2 & 0.58 & 0.05 \\
\hline 21 & 0.772 & 0.009 & 29.7 & 0.2 & 0.62 & 0.05 \\
\hline 25 & 0.6 & 0.01 & 30.3 & 0.4 & 0.70 & 0.05 \\
\hline 26 & 0.606 & 0.01 & 33.9 & 0.4 & 0.78 & 0.05 \\
\hline 27 & 0.607 & 0.015 & 32.7 & 0.5 & 0.95 & 0.05 \\
\hline 28 & 0.595 & 0.013 & 33.1 & 0.5 & 0.77 & 0.05 \\
\hline 29 & 0.59 & 0.012 & 34.5 & 0.5 & 0.80 & 0.05 \\
\hline 30 & 0.587 & 0.009 & 33.7 & 0.4 & 0.79 & 0.05 \\
\hline 31 & 0.607 & 0.018 & 33.5 & 0.6 & 0.84 & 0.05 \\
\hline 32 & 0.573 & 0.031 & 32.0 & 1.3 & 0.82 & 0.05 \\
\hline 33 & 0.577 & 0.025 & 30.7 & 1.0 & 0.73 & 0.05 \\
\hline 35 & 0.557 & 0.024 & 33.6 & 1.1 & 0.74 & 0.05 \\
\hline 37 & 0.647 & 0.037 & 36.6 & 1.7 & 0.81 & 0.05 \\
\hline 38 & 0.632 & 0.034 & 37.2 & 1.5 & 0.76 & 0.05 \\
\hline 41 & 0.596 & 0.017 & 20.3 & 0.6 & 0.79 & 0.05 \\
\hline 42 & 0.595 & 0.014 & 30.1 & 0.5 & 0.75 & 0.05 \\
\hline
\end{tabular}




\begin{tabular}{|c|c|c|c|c|c|c|}
\hline 43 & 0.602 & 0.009 & 29.4 & 0.3 & 0.38 & 0.05 \\
\hline 45 & 0.603 & 0.01 & 31.4 & 0.4 & 0.64 & 0.05 \\
\hline 46 & 0.562 & 0.005 & 29.0 & 0.2 & 0.52 & 0.05 \\
\hline 47 & 0.539 & 0.006 & 26.2 & 0.2 & 0.54 & 0.05 \\
\hline 48 & 0.562 & 0.007 & 27.5 & 0.2 & 0.53 & 0.05 \\
\hline 49 & 0.624 & 0.017 & 28.6 & 0.5 & 0.60 & 0.05 \\
\hline 50 & 0.578 & 0.005 & 28.3 & 0.2 & 0.69 & 0.05 \\
\hline 52 & 0.58 & 0.008 & 28.9 & 0.3 & 0.73 & 0.05 \\
\hline 53 & 0.613 & 0.013 & 31.8 & 0.5 & 0.74 & 0.05 \\
\hline 54 & 0.602 & 0.008 & 31.1 & 0.3 & 0.70 & 0.05 \\
\hline 55 & 0.601 & 0.01 & 30.7 & 0.4 & 0.77 & 0.05 \\
\hline 56 & 0.607 & 0.009 & 30.3 & 0.3 & 0.69 & 0.05 \\
\hline 57 & 0.58 & 0.007 & 30.9 & 0.3 & 0.77 & 0.05 \\
\hline 60 & 0.573 & 0.015 & 30.1 & 0.6 & 0.82 & 0.05 \\
\hline 61 & 0.612 & 0.01 & 30.7 & 0.4 & 0.72 & 0.05 \\
\hline 62 & 0.603 & 0.009 & 32.2 & 0.3 & 0.77 & 0.05 \\
\hline 63 & 0.614 & 0.014 & 32.4 & 0.5 & 0.80 & 0.05 \\
\hline 64 & 0.616 & 0.011 & 32.8 & 0.5 & 0.78 & 0.05 \\
\hline 65 & 0.641 & 0.01 & 33.7 & 0.4 & 0.81 & 0.05 \\
\hline 66 & 0.622 & 0.012 & 31.9 & 0.4 & 0.80 & 0.05 \\
\hline 67 & 0.615 & 0.014 & 32.1 & 0.5 & 0.84 & 0.05 \\
\hline 68 & 0.606 & 0.012 & 33.5 & 0.5 & 0.75 & 0.05 \\
\hline 69 & 0.628 & 0.012 & 30.8 & 0.4 & 0.81 & 0.05 \\
\hline 70 & 0.611 & 0.013 & 32.0 & 0.5 & 0.80 & 0.05 \\
\hline 71 & 0.598 & 0.014 & 31.3 & 0.5 & 0.79 & 0.05 \\
\hline 72 & 0.621 & 0.014 & 31.0 & 0.5 & 0.75 & 0.05 \\
\hline 73 & 0.621 & 0.012 & 32.2 & 0.4 & 0.81 & 0.05 \\
\hline 75 & 0.622 & 0.011 & 32.6 & 0.4 & 0.57 & 0.05 \\
\hline 78 & 0.614 & 0.009 & 32.5 & 0.4 & 0.70 & 0.05 \\
\hline 79 & 0.649 & 0.014 & 31.3 & 0.5 & 0.67 & 0.05 \\
\hline 82 & 0.618 & 0.026 & 31.0 & 0.9 & 0.69 & 0.05 \\
\hline
\end{tabular}




\begin{tabular}{|c|c|c|c|c|c|c|}
\hline 83 & 0.629 & 0.014 & 29.6 & 0.5 & 0.69 & 0.05 \\
\hline 84 & 0.628 & 0.013 & 30.2 & 0.4 & 0.74 & 0.05 \\
\hline 85 & 0.626 & 0.012 & 30.0 & 0.4 & 0.75 & 0.05 \\
\hline 86 & 0.595 & 0.01 & 31.5 & 0.4 & 0.77 & 0.05 \\
\hline 88 & 0.625 & 0.016 & 32.9 & 0.6 & 0.83 & 0.05 \\
\hline 89 & 0.623 & 0.015 & 34.0 & 0.6 & 0.92 & 0.05 \\
\hline 90 & 0.581 & 0.013 & 33.6 & 0.6 & 0.89 & 0.05 \\
\hline 92 & 0.583 & 0.021 & 31.2 & 0.8 & 0.77 & 0.05 \\
\hline 93 & 0.603 & 0.026 & 32.1 & 1.0 & 0.85 & 0.05 \\
\hline 94 & 0.534 & 0.02 & 30.0 & 0.8 & 0.74 & 0.05 \\
\hline 95 & 0.562 & 0.014 & 31.0 & 0.6 & 0.74 & 0.05 \\
\hline 96 & 0.57 & 0.017 & 29.3 & 0.6 & 0.74 & 0.05 \\
\hline 97 & 0.6 & 0.015 & 29.6 & 0.5 & 0.73 & 0.05 \\
\hline 99 & 0.641 & 0.016 & 30.8 & 0.5 & 0.77 & 0.05 \\
\hline 101 & 0.632 & 0.028 & 37.0 & 1.2 & 0.75 & 0.05 \\
\hline 102 & 0.579 & 0.02 & 35.6 & 0.9 & 0.88 & 0.05 \\
\hline 104 & 0.582 & 0.02 & 34.8 & 0.9 & 0.65 & 0.05 \\
\hline 106 & 0.555 & 0.016 & 36.5 & 0.8 & 0.84 & 0.05 \\
\hline 109 & 0.564 & 0.014 & 35.7 & 0.6 & 0.79 & 0.05 \\
\hline 112 & 0.564 & 0.013 & 33.9 & 0.6 & 0.81 & 0.05 \\
\hline 113 & 0.582 & 0.031 & 33.6 & 1.4 & 0.96 & 0.05 \\
\hline 115 & 0.578 & 0.016 & 35.1 & 0.7 & 0.76 & 0.05 \\
\hline 116 & 0.573 & 0.008 & 34.7 & 0.4 & 0.77 & 0.05 \\
\hline 117 & 0.576 & 0.01 & 33.9 & 0.4 & 0.79 & 0.05 \\
\hline 118 & 0.554 & 0.01 & 32.9 & 0.5 & 0.78 & 0.05 \\
\hline 119 & 0.635 & 0.008 & 30.3 & 0.3 & 0.44 & 0.05 \\
\hline 120 & 0.617 & 0.008 & 28.6 & 0.2 & 0.45 & 0.05 \\
\hline 121 & 0.623 & 0.007 & 30.6 & 0.3 & 0.69 & 0.05 \\
\hline 122 & 0.608 & 0.008 & 31.2 & 0.3 & 0.73 & 0.05 \\
\hline 123 & 0.722 & 0.04 & 37.3 & 1.5 & 0.71 & 0.05 \\
\hline 126 & 0.588 & 0.009 & 32.3 & 0.4 & 0.80 & 0.05 \\
\hline
\end{tabular}




$\begin{array}{lllllll}127 & 0.612 & 0.012 & 34.2 & 0.5 & 0.79 & 0.05 \\ 128 & 0.608 & 0.012 & 35.5 & 0.5 & 0.82 & 0.05 \\ 129 & 0.567 & 0.008 & 33.9 & 0.3 & 0.83 & 0.05 \\ 130 & 0.582 & 0.015 & 33.7 & 0.7 & 0.79 & 0.05 \\ 131 & 0.566 & 0.013 & 34.4 & 0.6 & 0.89 & 0.05 \\ 132 & 0.568 & 0.016 & 35.5 & 0.7 & 0.80 & 0.05 \\ 133 & 0.539 & 0.019 & 33.4 & 0.8 & 0.91 & 0.05 \\ 134 & 0.648 & 0.033 & 35.3 & 1.3 & 0.69 & 0.05 \\ 135 & 0.625 & 0.017 & 33.2 & 0.6 & 0.77 & 0.05 \\ 136 & 0.572 & 0.016 & 33.7 & 0.7 & 0.77 & 0.05 \\ 137 & 0.577 & 0.016 & 33.8 & 0.7 & 0.81 & 0.05 \\ 138 & 0.597 & 0.016 & 35.1 & 0.7 & 0.79 & 0.05 \\ 139 & 0.586 & 0.013 & 32.5 & 0.6 & 0.77 & 0.05 \\ 140 & 0.596 & 0.016 & 35.6 & 0.7 & 0.73 & 0.05 \\ 143 & 0.649 & 0.022 & 35.3 & 0.9 & 0.71 & 0.05 \\ 144 & 0.579 & 0.006 & 31.0 & 0.2 & 0.80 & 0.05 \\ 146 & 0.892 & 0.013 & 23.8 & 0.2 & 0.52 & 0.05\end{array}$


$\beta$-chain at $34{ }^{\circ} \mathrm{C}$ and at $14.1 \mathrm{~T}$ in the CO form

\begin{tabular}{|c|c|c|c|c|c|c|}
\hline $\begin{array}{c}\text { Residue } \\
\text { No. }\end{array}$ & $\mathbf{R}_{1}$ & $R_{1}$ error & $\mathbf{R}_{\mathbf{2}}$ & $\mathbf{R}_{\mathbf{2}}$ error & NOE & NOE erro \\
\hline 3 & 0.552 & 0.005 & 26.1 & 0.2 & 0.33 & 0.05 \\
\hline 4 & 0.472 & 0.004 & 31.2 & 0.2 & 0.66 & 0.05 \\
\hline 6 & 0.461 & 0.013 & 34.5 & 0.5 & 0.72 & 0.05 \\
\hline 7 & 0.472 & 0.007 & 35.2 & 0.3 & 0.73 & 0.05 \\
\hline 8 & 0.476 & 0.006 & 33.7 & 0.3 & 0.78 & 0.05 \\
\hline 9 & 0.477 & 0.005 & 32.8 & 0.2 & 0.86 & 0.05 \\
\hline 10 & 0.482 & 0.005 & 36.1 & 0.3 & 0.79 & 0.05 \\
\hline 12 & 0.478 & 0.005 & 33.8 & 0.3 & 0.79 & 0.05 \\
\hline 13 & 0.484 & 0.007 & 34.4 & 0.3 & 0.79 & 0.05 \\
\hline 16 & 0.471 & 0.007 & 33.3 & 0.3 & 0.83 & 0.05 \\
\hline 18 & 0.457 & 0.005 & 33.9 & 0.3 & 0.80 & 0.05 \\
\hline 19 & 0.488 & 0.006 & 32.2 & 0.3 & 0.65 & 0.05 \\
\hline 20 & 0.565 & 0.006 & 31.5 & 0.2 & 0.62 & 0.05 \\
\hline 21 & 0.631 & 0.006 & 31.9 & 0.2 & 0.58 & 0.05 \\
\hline 24 & 0.509 & 0.009 & 37.0 & 0.4 & 0.75 & 0.05 \\
\hline 25 & 0.493 & 0.007 & 33.9 & 0.3 & 0.72 & 0.05 \\
\hline 26 & 0.487 & 0.007 & 36.7 & 0.3 & 0.79 & 0.05 \\
\hline 28 & 0.468 & 0.007 & 35.0 & 0.4 & 0.85 & 0.05 \\
\hline 29 & 0.463 & 0.008 & 35.9 & 0.4 & 0.88 & 0.05 \\
\hline 30 & 0.464 & 0.007 & 34.0 & 0.3 & 0.83 & 0.05 \\
\hline 31 & 0.473 & 0.006 & 35.7 & 0.3 & 0.79 & 0.05 \\
\hline 32 & 0.444 & 0.016 & 34.5 & 1.0 & 0.77 & 0.05 \\
\hline 33 & 0.45 & 0.014 & 34.6 & 0.8 & 0.82 & 0.05 \\
\hline 35 & 0.437 & 0.015 & 35.0 & 0.8 & 0.88 & 0.05 \\
\hline 37 & 0.469 & 0.025 & 39.5 & 1.5 & 0.82 & 0.05 \\
\hline 38 & 0.512 & 0.019 & 36.9 & 0.9 & 0.84 & 0.05 \\
\hline 41 & 0.48 & 0.011 & 23.5 & 0.5 & 0.78 & 0.05 \\
\hline 42 & 0.456 & 0.009 & 32.1 & 0.4 & 0.78 & 0.05 \\
\hline
\end{tabular}




\begin{tabular}{|c|c|c|c|c|c|c|}
\hline 43 & 0.479 & 0.006 & 32.4 & 0.3 & 0.43 & 0.05 \\
\hline 45 & 0.486 & 0.007 & 34.8 & 0.3 & 0.61 & 0.05 \\
\hline 46 & 0.455 & 0.004 & 32.9 & 0.2 & 0.51 & 0.05 \\
\hline 47 & 0.428 & 0.004 & 29.0 & 0.2 & 0.53 & 0.05 \\
\hline 48 & 0.446 & 0.004 & 29.9 & 0.2 & 0.58 & 0.05 \\
\hline 49 & 0.513 & 0.01 & 30.7 & 0.4 & 0.61 & 0.05 \\
\hline 50 & 0.471 & 0.003 & 31.1 & 0.2 & 0.68 & 0.05 \\
\hline 52 & 0.464 & 0.005 & 32.1 & 0.2 & 0.71 & 0.05 \\
\hline 53 & 0.483 & 0.007 & 35.5 & 0.3 & 0.80 & 0.05 \\
\hline 54 & 0.462 & 0.005 & 32.9 & 0.2 & 0.79 & 0.05 \\
\hline 55 & 0.482 & 0.006 & 33.3 & 0.3 & 0.80 & 0.05 \\
\hline 56 & 0.498 & 0.006 & 32.4 & 0.3 & 0.75 & 0.05 \\
\hline 57 & 0.458 & 0.004 & 34.3 & 0.2 & 0.81 & 0.05 \\
\hline 60 & 0.463 & 0.009 & 33.1 & 0.5 & 0.78 & 0.05 \\
\hline 61 & 0.481 & 0.007 & 32.7 & 0.3 & 0.77 & 0.05 \\
\hline 62 & 0.485 & 0.006 & 34.6 & 0.3 & 0.79 & 0.05 \\
\hline 63 & 0.489 & 0.008 & 35.6 & 0.4 & 0.82 & 0.05 \\
\hline 64 & 0.479 & 0.007 & 35.6 & 0.4 & 0.82 & 0.05 \\
\hline 65 & 0.494 & 0.006 & 35.5 & 0.4 & 0.87 & 0.05 \\
\hline 66 & 0.482 & 0.007 & 34.2 & 0.4 & 0.78 & 0.05 \\
\hline 67 & 0.471 & 0.01 & 34.4 & 0.4 & 0.78 & 0.05 \\
\hline 69 & 0.49 & 0.008 & 34.2 & 0.4 & 0.82 & 0.05 \\
\hline 70 & 0.482 & 0.008 & 35.6 & 0.3 & 0.97 & 0.05 \\
\hline 71 & 0.466 & 0.009 & 32.9 & 0.4 & 0.81 & 0.05 \\
\hline 72 & 0.468 & 0.008 & 33.7 & 0.4 & 0.80 & 0.05 \\
\hline 73 & 0.482 & 0.008 & 35.5 & 0.4 & 0.81 & 0.05 \\
\hline 75 & 0.483 & 0.005 & 35.3 & 0.2 & 0.83 & 0.05 \\
\hline 76 & 0.49 & 0.008 & 31.9 & 0.4 & 0.78 & 0.05 \\
\hline 79 & 0.519 & 0.01 & 34.3 & 0.4 & 0.70 & 0.05 \\
\hline 82 & 0.491 & 0.017 & 34.4 & 0.7 & 0.68 & 0.05 \\
\hline 83 & 0.515 & 0.009 & 32.7 & 0.4 & 0.71 & 0.05 \\
\hline
\end{tabular}




\begin{tabular}{|c|c|c|c|c|c|c|}
\hline 84 & 0.512 & 0.01 & 32.3 & 0.4 & 0.74 & 0.05 \\
\hline 85 & 0.493 & 0.007 & 32.2 & 0.4 & 0.72 & 0.05 \\
\hline 86 & 0.47 & 0.006 & 36.0 & 0.3 & 0.78 & 0.05 \\
\hline 88 & 0.512 & 0.01 & 36.6 & 0.5 & 0.80 & 0.05 \\
\hline 89 & 0.488 & 0.01 & 37.2 & 0.5 & 0.87 & 0.05 \\
\hline 90 & 0.467 & 0.009 & 36.4 & 0.5 & 0.78 & 0.05 \\
\hline 91 & 0.466 & 0.004 & 33.1 & 0.2 & 0.36 & 0.05 \\
\hline 92 & 0.465 & 0.014 & 34.1 & 0.8 & 0.85 & 0.05 \\
\hline 93 & 0.46 & 0.015 & 33.8 & 0.7 & 0.74 & 0.05 \\
\hline 94 & 0.425 & 0.012 & 32.4 & 0.7 & 0.79 & 0.05 \\
\hline 95 & 0.435 & 0.008 & 33.3 & 0.5 & 0.91 & 0.05 \\
\hline 96 & 0.444 & 0.01 & 31.7 & 0.5 & 0.76 & 0.05 \\
\hline 97 & 0.475 & 0.008 & 32.9 & 0.5 & 0.84 & 0.05 \\
\hline 99 & 0.525 & 0.011 & 34.6 & 0.5 & 0.81 & 0.05 \\
\hline 101 & 0.477 & 0.019 & 39.5 & 1.0 & 0.94 & 0.05 \\
\hline 102 & 0.448 & 0.012 & 36.1 & 0.6 & 0.85 & 0.05 \\
\hline 104 & 0.465 & 0.012 & 36.3 & 0.6 & 0.77 & 0.05 \\
\hline 106 & 0.442 & 0.01 & 36.0 & 0.5 & 0.82 & 0.05 \\
\hline 109 & 0.453 & 0.009 & 39.3 & 0.6 & 0.82 & 0.05 \\
\hline 110 & 0.467 & 0.029 & 38.5 & 1.5 & 0.84 & 0.05 \\
\hline 112 & 0.447 & 0.008 & 35.3 & 0.5 & 0.81 & 0.05 \\
\hline 113 & 0.474 & 0.021 & 36.5 & 1.0 & 0.44 & 0.05 \\
\hline 115 & 0.457 & 0.009 & 37.2 & 0.6 & 0.74 & 0.05 \\
\hline 116 & 0.449 & 0.005 & 35.5 & 0.3 & 0.83 & 0.05 \\
\hline 117 & 0.451 & 0.006 & 35.3 & 0.3 & 0.83 & 0.05 \\
\hline 118 & 0.44 & 0.007 & 33.9 & 0.3 & 0.76 & 0.05 \\
\hline 119 & 0.512 & 0.005 & 32.3 & 0.2 & 0.44 & 0.05 \\
\hline 120 & 0.5 & 0.005 & 32.2 & 0.2 & 0.55 & 0.05 \\
\hline 121 & 0.493 & 0.005 & 33.0 & 0.2 & 0.72 & 0.05 \\
\hline 122 & 0.489 & 0.005 & 34.5 & 0.2 & 0.76 & 0.05 \\
\hline 123 & 0.546 & 0.022 & 38.2 & 1.1 & 0.75 & 0.05 \\
\hline
\end{tabular}




$\begin{array}{lllllll}126 & 0.456 & 0.005 & 35.4 & 0.3 & 0.83 & 0.05 \\ 127 & 0.474 & 0.009 & 36.5 & 0.4 & 0.89 & 0.05 \\ 128 & 0.467 & 0.008 & 37.9 & 0.4 & 0.82 & 0.05 \\ 129 & 0.455 & 0.005 & 36.5 & 0.3 & 0.85 & 0.05 \\ 130 & 0.454 & 0.009 & 35.0 & 0.5 & 0.80 & 0.05 \\ 131 & 0.464 & 0.01 & 36.2 & 0.5 & 0.87 & 0.05 \\ 132 & 0.457 & 0.011 & 38.6 & 0.6 & 0.81 & 0.05 \\ 133 & 0.449 & 0.011 & 34.8 & 0.6 & 0.87 & 0.05 \\ 134 & 0.454 & 0.022 & 38.4 & 1.2 & 0.91 & 0.05 \\ 135 & 0.495 & 0.013 & 37.9 & 0.7 & 0.84 & 0.05 \\ 136 & 0.451 & 0.012 & 36.4 & 0.7 & 0.85 & 0.05 \\ 137 & 0.462 & 0.011 & 37.6 & 0.6 & 0.82 & 0.05 \\ 138 & 0.484 & 0.01 & 36.8 & 0.5 & 0.71 & 0.05 \\ 139 & 0.464 & 0.009 & 34.5 & 0.4 & 0.78 & 0.05 \\ 140 & 0.479 & 0.01 & 37.6 & 0.6 & 0.82 & 0.05 \\ 142 & 0.477 & 0.008 & 34.8 & 0.4 & 0.77 & 0.05 \\ 143 & 0.491 & 0.013 & 37.2 & 0.7 & 0.82 & 0.05 \\ 144 & 0.448 & 0.003 & 32.4 & 0.2 & 0.84 & 0.05 \\ 146 & 0.748 & 0.009 & 26.9 & 0.2 & 0.57 & 0.05\end{array}$

END OF THE LIST 
Table 3S. Amino Acid Residues in $\mathrm{Hb}$ A Selected for Correlation Time Determination Units: $R_{2} / R_{1}$ and $d R_{2} / R_{1}$, none; $R_{1} R_{2}$ and $d R_{1} R_{2}: s^{-2}$

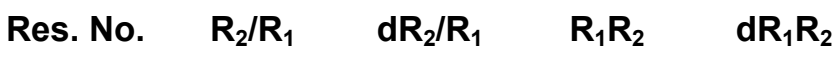

\begin{tabular}{ccccc} 
Deoxy & $\alpha$-chain & $\mathbf{2 9}{ }^{\circ} \mathrm{C}$ & $\mathbf{5 0 0} \mathbf{M H z}$ & \\
& & & & \\
5 & 77.46 & 2.71 & 17.92 & 0.63 \\
6 & 75.96 & 2.07 & 19.30 & 0.53 \\
8 & 74.45 & 2.38 & 18.02 & 0.58 \\
9 & 76.06 & 2.34 & 19.55 & 0.60 \\
10 & 79.14 & 2.09 & 19.24 & 0.51 \\
11 & 77.07 & 2.19 & 20.05 & 0.57 \\
12 & 78.13 & 2.12 & 19.53 & 0.53 \\
14 & 77.23 & 2.75 & 19.38 & 0.69 \\
15 & 77.81 & 4.03 & 20.32 & 1.05 \\
16 & 75.27 & 1.72 & 19.04 & 0.44 \\
23 & 74.35 & 1.43 & 18.37 & 0.35 \\
28 & 77.44 & 2.80 & 19.59 & 0.71 \\
29 & 79.61 & 6.98 & 19.19 & 1.68 \\
30 & 79.07 & 3.15 & 18.68 & 0.74 \\
33 & 78.56 & 3.77 & 18.94 & 0.91 \\
34 & 76.29 & 2.51 & 18.54 & 0.61 \\
36 & 79.26 & 1.66 & 18.34 & 0.38 \\
38 & 71.57 & 1.72 & 20.33 & 0.49 \\
41 & 76.73 & 4.25 & 18.50 & 1.02 \\
46 & 80.75 & 2.47 & 19.23 & 0.59 \\
54 & 70.67 & 2.03 & 19.26 & 0.55 \\
\hline 6 & 76.25 & 1.99 & 19.52 & 0.51
\end{tabular}




$\begin{array}{rllll}57 & 74.42 & 2.05 & 19.20 & 0.53 \\ 60 & 79.51 & 4.11 & 18.62 & 0.96 \\ 67 & 74.60 & 2.80 & 20.17 & 0.76 \\ 69 & 75.72 & 2.50 & 19.46 & 0.64 \\ 70 & 73.31 & 2.05 & 18.84 & 0.53 \\ 71 & 76.29 & 2.23 & 20.23 & 0.59 \\ 73 & 79.87 & 1.85 & 18.71 & 0.43 \\ 81 & 72.36 & 2.18 & 19.95 & 0.60 \\ 104 & 76.50 & 3.48 & 19.05 & 0.87 \\ 109 & 81.28 & 2.87 & 19.52 & 0.69 \\ 113 & 75.88 & 1.31 & 19.12 & 0.33 \\ 117 & 76.25 & 1.77 & 18.68 & 0.43 \\ 118 & 70.65 & 1.15 & 17.66 & 0.29 \\ 120 & 78.54 & 1.63 & 17.65 & 0.37 \\ 121 & 80.79 & 2.85 & 19.00 & 0.67 \\ 124 & 82.01 & 2.36 & 18.19 & 0.52 \\ 126 & 81.82 & 2.05 & 20.13 & 0.50 \\ 127 & 78.84 & 1.66 & 18.70 & 0.39 \\ 131 & 80.16 & 2.47 & 17.94 & 0.55 \\ 132 & 81.25 & 3.08 & 19.75 & 0.75 \\ 134 & 80.18 & 2.98 & 18.17 & 0.67 \\ 139 & 75.97 & 2.89 & 19.22 & 0.73 \\ 140 & 77.69 & 3.94 & 19.89 & 1.01 \\ 141 & 72.17 & 1.04 & 17.97 & 0.26\end{array}$

Deoxy $\quad \alpha$-chain $\quad 29^{\circ} \mathrm{C} \quad 600 \mathrm{MHz}$

$\begin{array}{crrrr}6 & 103.91 & 3.30 & 15.97 & 0.51 \\ 9 & 111.76 & 3.10 & 17.17 & 0.48 \\ 10 & 110.16 & 2.93 & 16.33 & 0.44\end{array}$




\begin{tabular}{|c|c|c|c|c|}
\hline 11 & 105.54 & 2.80 & 16.55 & 0.44 \\
\hline 12 & 113.83 & 2.69 & 16.96 & 0.40 \\
\hline 14 & 110.47 & 4.17 & 15.37 & 0.58 \\
\hline 15 & 101.20 & 2.65 & 16.93 & 0.44 \\
\hline 22 & 109.73 & 5.72 & 16.35 & 0.85 \\
\hline 23 & 103.18 & 1.91 & 16.34 & 0.30 \\
\hline 25 & 115.74 & 4.63 & 17.51 & 0.70 \\
\hline 26 & 113.69 & 4.23 & 17.83 & 0.66 \\
\hline 28 & 112.69 & 4.45 & 17.49 & 0.69 \\
\hline 30 & 112.69 & 4.89 & 15.85 & 0.69 \\
\hline 32 & 109.15 & 4.37 & 17.55 & 0.70 \\
\hline 34 & 103.32 & 3.24 & 16.12 & 0.51 \\
\hline 41 & 116.38 & 12.04 & 16.10 & 1.67 \\
\hline 46 & 111.15 & 4.16 & 16.22 & 0.61 \\
\hline 54 & 101.40 & 2.98 & 17.05 & 0.50 \\
\hline 56 & 104.23 & 2.37 & 16.51 & 0.38 \\
\hline 57 & 101.63 & 3.17 & 16.51 & 0.52 \\
\hline 60 & 104.06 & 7.62 & 15.83 & 1.16 \\
\hline 67 & 103.95 & 4.76 & 17.05 & 0.78 \\
\hline 69 & 105.80 & 4.03 & 17.01 & 0.65 \\
\hline 71 & 104.63 & 2.67 & 17.67 & 0.45 \\
\hline 73 & 105.04 & 2.50 & 16.72 & 0.40 \\
\hline 89 & 108.84 & 12.58 & 17.59 & 2.03 \\
\hline 96 & 114.98 & 4.63 & 16.69 & 0.67 \\
\hline 104 & 108.41 & 6.28 & 15.41 & 0.89 \\
\hline 108 & 113.74 & 6.24 & 16.25 & 0.89 \\
\hline 109 & 114.43 & 5.77 & 17.41 & 0.88 \\
\hline 113 & 101.46 & 2.21 & 16.64 & 0.36 \\
\hline 117 & 103.89 & 2.34 & 16.71 & 0.38 \\
\hline 118 & 99.19 & 1.70 & 15.40 & 0.26 \\
\hline
\end{tabular}




$\begin{array}{rrrrr}123 & 115.65 & 2.22 & 17.95 & 0.34 \\ 124 & 114.76 & 3.26 & 15.37 & 0.44 \\ 131 & 112.08 & 3.78 & 15.43 & 0.52 \\ 132 & 116.23 & 5.37 & 17.32 & 0.80 \\ 134 & 115.07 & 5.86 & 16.27 & 0.83 \\ 140 & 109.36 & 6.72 & 17.67 & 1.09\end{array}$

Deoxy $\alpha$-chain $\quad 34^{\circ} \mathrm{C} \quad 500 \mathrm{MHz}$

$\begin{array}{lllll}6 & 60.31 & 1.76 & 18.44 & 0.54 \\ 8 & 58.42 & 1.20 & 18.12 & 0.37 \\ 9 & 60.73 & 1.32 & 19.59 & 0.43 \\ 10 & 61.30 & 1.20 & 19.43 & 0.38 \\ 11 & 59.26 & 1.17 & 19.19 & 0.38 \\ 12 & 61.59 & 1.16 & 19.66 & 0.37 \\ 13 & 63.96 & 1.09 & 18.58 & 0.32 \\ 14 & 60.18 & 1.61 & 19.42 & 0.52 \\ 15 & 59.93 & 2.24 & 19.20 & 0.72 \\ 16 & 57.11 & 0.95 & 18.49 & 0.31 \\ 23 & 56.74 & 0.89 & 18.24 & 0.29 \\ 25 & 59.87 & 1.37 & 20.42 & 0.47 \\ 26 & 64.07 & 1.48 & 20.17 & 0.46 \\ 27 & 62.56 & 1.07 & 18.65 & 0.32 \\ 28 & 60.63 & 1.54 & 19.77 & 0.50 \\ 29 & 60.93 & 3.53 & 17.90 & 1.04 \\ 30 & 61.71 & 1.72 & 18.20 & 0.51 \\ 33 & 61.08 & 2.00 & 20.33 & 0.67 \\ 34 & 59.19 & 1.35 & 18.89 & 0.43 \\ 36 & 61.10 & 1.02 & 18.62 & 0.31 \\ 40 & 55.96 & 1.37 & 20.35 & 0.50\end{array}$




\begin{tabular}{|c|c|c|c|c|}
\hline 41 & 57.50 & 1.95 & 19.34 & 0.65 \\
\hline 43 & 57.13 & 2.55 & 17.85 & 0.80 \\
\hline 46 & 61.88 & 1.39 & 19.06 & 0.43 \\
\hline 54 & 59.56 & 2.99 & 19.97 & 1.00 \\
\hline 56 & 57.99 & 1.12 & 19.04 & 0.37 \\
\hline 57 & 57.66 & 1.13 & 19.20 & 0.38 \\
\hline 60 & 57.17 & 1.89 & 18.19 & 0.60 \\
\hline 67 & 58.67 & 1.62 & 19.60 & 0.54 \\
\hline 69 & 59.39 & 1.34 & 19.77 & 0.45 \\
\hline 70 & 56.83 & 1.15 & 18.79 & 0.38 \\
\hline 71 & 58.89 & 0.92 & 20.22 & 0.32 \\
\hline 73 & 59.06 & 0.98 & 18.52 & 0.31 \\
\hline 74 & 55.64 & 1.01 & 19.30 & 0.35 \\
\hline 75 & 58.05 & 1.08 & 17.95 & 0.33 \\
\hline 81 & 56.40 & 1.27 & 19.50 & 0.44 \\
\hline 89 & 62.48 & 3.12 & 18.69 & 0.93 \\
\hline 104 & 59.46 & 1.99 & 18.58 & 0.62 \\
\hline 108 & 61.53 & 2.63 & 18.82 & 0.80 \\
\hline 111 & 62.92 & 1.08 & 19.03 & 0.33 \\
\hline 113 & 59.11 & 0.81 & 18.60 & 0.25 \\
\hline 117 & 58.17 & 1.09 & 18.77 & 0.35 \\
\hline 118 & 55.91 & 0.72 & 18.36 & 0.24 \\
\hline 120 & 60.38 & 0.87 & 18.33 & 0.26 \\
\hline 121 & 63.17 & 1.53 & 19.04 & 0.46 \\
\hline 124 & 62.89 & 1.31 & 19.02 & 0.40 \\
\hline 127 & 63.55 & 1.48 & 19.36 & 0.45 \\
\hline 131 & 64.05 & 1.61 & 18.54 & 0.47 \\
\hline 134 & 62.71 & 1.71 & 18.83 & 0.51 \\
\hline 138 & 58.07 & 2.93 & 20.08 & 1.01 \\
\hline 140 & 59.01 & 2.15 & 20.20 & 0.73 \\
\hline
\end{tabular}




\begin{tabular}{|c|c|c|c|c|}
\hline Deoxy & $\alpha$-chain & $34^{\circ} \mathrm{C}$ & $600 \mathrm{MHz}$ & \\
\hline 6 & 82.97 & 2.55 & 14.78 & 0.45 \\
\hline 8 & 81.21 & 1.87 & 14.74 & 0.34 \\
\hline 9 & 89.42 & 2.08 & 16.61 & 0.39 \\
\hline 10 & 89.78 & 2.06 & 15.84 & 0.36 \\
\hline 11 & 82.84 & 1.86 & 16.04 & 0.36 \\
\hline 12 & 87.96 & 1.94 & 16.72 & 0.37 \\
\hline 14 & 84.69 & 2.84 & 15.08 & 0.51 \\
\hline 15 & 80.38 & 1.79 & 16.50 & 0.37 \\
\hline 16 & 78.64 & 1.69 & 15.93 & 0.34 \\
\hline 22 & 88.87 & 6.19 & 15.23 & 1.06 \\
\hline 23 & 79.57 & 1.43 & 15.69 & 0.28 \\
\hline 27 & 87.38 & 1.78 & 15.19 & 0.31 \\
\hline 28 & 90.42 & 3.06 & 16.72 & 0.57 \\
\hline 30 & 85.77 & 3.06 & 14.91 & 0.53 \\
\hline 32 & 85.07 & 2.78 & 16.62 & 0.54 \\
\hline 33 & 88.87 & 4.08 & 16.13 & 0.74 \\
\hline 34 & 83.22 & 2.21 & 15.24 & 0.40 \\
\hline 40 & 76.19 & 2.68 & 16.76 & 0.59 \\
\hline 41 & 90.77 & 6.89 & 14.74 & 1.12 \\
\hline 46 & 86.13 & 2.63 & 15.93 & 0.49 \\
\hline 53 & 83.15 & 11.43 & 14.67 & 2.02 \\
\hline 54 & 84.85 & 2.22 & 15.84 & 0.41 \\
\hline 56 & 81.58 & 1.77 & 15.87 & 0.34 \\
\hline 57 & 80.03 & 2.07 & 15.56 & 0.40 \\
\hline 60 & 77.98 & 4.39 & 14.82 & 0.84 \\
\hline 67 & 82.09 & 3.02 & 16.18 & 0.60 \\
\hline 69 & 85.63 & 2.50 & 16.58 & 0.48 \\
\hline
\end{tabular}




$\begin{array}{lllll}70 & 80.69 & 1.85 & 15.13 & 0.35 \\ 73 & 84.29 & 1.91 & 15.51 & 0.35 \\ 79 & 82.45 & 1.85 & 15.60 & 0.35 \\ 81 & 77.01 & 2.38 & 16.01 & 0.49 \\ 89 & 90.44 & 7.76 & 16.11 & 1.38 \\ 96 & 91.86 & 2.59 & 16.13 & 0.45 \\ 104 & 87.33 & 4.20 & 14.82 & 0.71 \\ 108 & 90.34 & 4.34 & 15.41 & 0.74 \\ 113 & 83.40 & 1.57 & 15.49 & 0.29 \\ 117 & 82.32 & 1.58 & 15.72 & 0.30 \\ 118 & 79.23 & 1.18 & 14.99 & 0.22 \\ 120 & 86.51 & 1.40 & 15.12 & 0.24 \\ 124 & 90.03 & 2.17 & 14.91 & 0.36 \\ 127 & 87.36 & 2.31 & 15.41 & 0.41 \\ 135 & 92.21 & 3.73 & 15.28 & 0.62 \\ 138 & 82.20 & 6.67 & 16.79 & 1.36 \\ 140 & 85.47 & 3.95 & 15.80 & 0.73 \\ 141 & 79.26 & 0.92 & 14.86 & 0.17\end{array}$

\section{Deoxy $\quad \beta$-chain $\quad 29^{\circ} \mathrm{C} \quad 500 \mathrm{MHz}$}

$\begin{array}{lllll}4 & 66.21 & 2.98 & 18.74 & 0.84 \\ 6 & 69.85 & 1.62 & 18.10 & 0.42 \\ 7 & 67.65 & 2.75 & 19.51 & 0.79 \\ 8 & 68.58 & 2.62 & 19.34 & 0.74 \\ 9 & 67.24 & 1.77 & 18.53 & 0.49 \\ 10 & 68.71 & 1.79 & 19.37 & 0.50 \\ 11 & 70.88 & 2.14 & 18.87 & 0.57 \\ 12 & 66.23 & 2.54 & 19.17 & 0.73 \\ 13 & 63.81 & 2.09 & 18.47 & 0.61\end{array}$




\begin{tabular}{|c|c|c|c|}
\hline 15 & 72.02 & 4.43 & 19.55 \\
\hline 16 & 69.54 & 2.29 & 19.31 \\
\hline 17 & 71.93 & 2.65 & 19.15 \\
\hline 18 & 66.72 & 1.70 & 18.11 \\
\hline 23 & 65.60 & 2.43 & 19.49 \\
\hline 25 & 67.05 & 2.33 & 18.91 \\
\hline 26 & 67.31 & 2.53 & 19.63 \\
\hline 27 & 68.41 & 2.96 & 19.95 \\
\hline 29 & 71.31 & 3.02 & 19.73 \\
\hline 30 & 69.39 & 2.91 & 19.27 \\
\hline 31 & 67.93 & 6.83 & 18.58 \\
\hline 34 & 67.52 & 6.85 & 19.25 \\
\hline 35 & 70.59 & 4.60 & 19.68 \\
\hline 37 & 70.68 & 3.35 & 20.76 \\
\hline 39 & 68.26 & 2.74 & 20.57 \\
\hline 40 & 68.28 & 4.01 & 18.53 \\
\hline 42 & 66.68 & 5.44 & 18.31 \\
\hline 43 & 68.35 & 1.46 & 20.23 \\
\hline 45 & 70.36 & 3.17 & 19.47 \\
\hline 54 & 67.82 & 1.75 & 18.62 \\
\hline 55 & 64.99 & 1.88 & 18.67 \\
\hline 56 & 64.48 & 1.84 & 17.77 \\
\hline 59 & 67.88 & 1.92 & 20.38 \\
\hline 60 & 67.85 & 3.18 & 20.01 \\
\hline 61 & 67.16 & 1.86 & 19.80 \\
\hline 62 & 68.66 & 2.33 & 19.95 \\
\hline 65 & 69.40 & 4.63 & 20.09 \\
\hline 72 & 65.31 & 4.35 & 20.55 \\
\hline 74 & 62.39 & 2.72 & 19.01 \\
\hline 76 & 64.10 & 1.77 & 19.60 \\
\hline
\end{tabular}




$\begin{array}{lllll}78 & 69.12 & 2.63 & 18.91 & 0.72 \\ 80 & 63.55 & 1.76 & 18.60 & 0.52 \\ 82 & 66.41 & 3.00 & 19.44 & 0.88 \\ 83 & 63.41 & 3.02 & 18.90 & 0.90 \\ 85 & 65.03 & 3.51 & 18.75 & 1.01 \\ 86 & 68.12 & 3.62 & 17.86 & 0.95 \\ 87 & 64.69 & 3.92 & 18.31 & 1.11 \\ 94 & 65.38 & 5.89 & 19.42 & 1.75 \\ 101 & 68.39 & 2.86 & 19.94 & 0.83 \\ 115 & 72.28 & 4.05 & 20.69 & 1.16 \\ 116 & 71.37 & 1.84 & 19.60 & 0.51 \\ 118 & 71.07 & 3.21 & 18.85 & 0.85 \\ 121 & 66.65 & 1.47 & 19.29 & 0.43 \\ 126 & 71.11 & 2.05 & 19.30 & 0.56 \\ 127 & 72.06 & 2.55 & 20.47 & 0.72 \\ 128 & 72.59 & 2.57 & 20.39 & 0.72 \\ 133 & 68.79 & 3.62 & 19.98 & 1.05 \\ 136 & 70.81 & 2.80 & 18.42 & 0.73 \\ 137 & 71.47 & 2.53 & 20.38 & 0.72 \\ 139 & 70.76 & 3.17 & 17.76 & 0.79 \\ 140 & 71.35 & 4.24 & 18.12 & 1.08 \\ 144 & 67.12 & 1.31 & 18.15 & 0.35\end{array}$

Deoxy $\quad \beta$-chain $\quad 29^{\circ} \mathrm{C} \quad 600 \mathrm{MHz}$

$\begin{array}{lllll}4 & 90.58 & 4.11 & 15.90 & 0.72 \\ 6 & 91.66 & 1.90 & 15.41 & 0.32 \\ 7 & 92.52 & 3.10 & 16.01 & 0.54 \\ 8 & 94.45 & 3.49 & 16.35 & 0.60 \\ 9 & 90.90 & 1.96 & 15.50 & 0.33\end{array}$




\begin{tabular}{|c|c|c|c|c|}
\hline 10 & 90.40 & 2.55 & 16.48 & 0.46 \\
\hline 11 & 94.19 & 2.40 & 15.45 & 0.39 \\
\hline 12 & 85.33 & 2.41 & 16.00 & 0.45 \\
\hline 13 & 91.08 & 2.56 & 16.53 & 0.47 \\
\hline 15 & 100.62 & 5.21 & 16.83 & 0.87 \\
\hline 16 & 94.01 & 2.73 & 15.80 & 0.46 \\
\hline 17 & 98.79 & 3.09 & 15.81 & 0.49 \\
\hline 18 & 91.61 & 1.98 & 15.10 & 0.33 \\
\hline 23 & 91.44 & 3.03 & 16.05 & 0.53 \\
\hline 25 & 88.51 & 2.80 & 16.67 & 0.53 \\
\hline 26 & 90.59 & 2.94 & 16.06 & 0.52 \\
\hline 27 & 94.91 & 3.92 & 17.14 & 0.71 \\
\hline 29 & 101.27 & 4.07 & 15.40 & 0.62 \\
\hline 30 & 95.33 & 3.50 & 16.50 & 0.60 \\
\hline 31 & 100.34 & 9.97 & 15.58 & 1.55 \\
\hline 32 & 96.45 & 11.66 & 16.29 & 1.97 \\
\hline 33 & 89.02 & 4.42 & 16.00 & 0.79 \\
\hline 35 & 97.11 & 5.17 & 15.54 & 0.83 \\
\hline 37 & 93.15 & 3.93 & 16.67 & 0.70 \\
\hline 38 & 91.30 & 5.09 & 17.12 & 0.95 \\
\hline 40 & 92.55 & 5.37 & 16.09 & 0.93 \\
\hline 53 & 88.08 & 2.88 & 15.84 & 0.52 \\
\hline 54 & 93.06 & 2.09 & 15.19 & 0.34 \\
\hline 55 & 87.85 & 2.18 & 15.87 & 0.39 \\
\hline 56 & 82.64 & 1.78 & 14.79 & 0.32 \\
\hline 59 & 91.29 & 2.55 & 16.96 & 0.47 \\
\hline 60 & 87.61 & 3.51 & 17.35 & 0.70 \\
\hline 61 & 92.92 & 2.33 & 16.55 & 0.41 \\
\hline 62 & 90.90 & 2.92 & 16.50 & 0.53 \\
\hline 65 & 87.44 & 6.80 & 16.17 & 1.26 \\
\hline
\end{tabular}




$\begin{array}{rllll}74 & 88.01 & 3.58 & 16.35 & 0.66 \\ 76 & 86.66 & 2.13 & 16.63 & 0.41 \\ 78 & 92.51 & 2.68 & 16.55 & 0.48 \\ 80 & 89.21 & 2.16 & 15.81 & 0.38 \\ 82 & 91.33 & 4.23 & 16.65 & 0.77 \\ 83 & 85.62 & 3.80 & 15.76 & 0.70 \\ 85 & 86.55 & 4.16 & 16.30 & 0.78 \\ 86 & 89.52 & 4.62 & 15.64 & 0.81 \\ 87 & 87.70 & 5.19 & 15.47 & 0.92 \\ 94 & 92.10 & 9.47 & 14.81 & 1.52 \\ 101 & 91.28 & 4.12 & 16.72 & 0.76 \\ 105 & 95.48 & 7.94 & 16.60 & 1.38 \\ 108 & 101.55 & 3.52 & 17.07 & 0.59 \\ 115 & 99.16 & 4.65 & 16.75 & 0.79 \\ 116 & 101.37 & 2.21 & 16.46 & 0.36 \\ 118 & 95.27 & 3.20 & 16.01 & 0.54 \\ 121 & 88.10 & 1.74 & 16.21 & 0.32 \\ 122 & 84.53 & 2.01 & 15.34 & 0.36 \\ 126 & 97.97 & 2.25 & 15.44 & 0.36 \\ 127 & 101.27 & 3.03 & 17.44 & 0.52 \\ 128 & 99.76 & 3.04 & 16.53 & 0.50 \\ 136 & 97.78 & 3.42 & 15.02 & 0.53 \\ 137 & 99.27 & 3.40 & 16.77 & 0.57 \\ 143 & 95.76 & 5.65 & 16.97 & 1.00 \\ 144 & 87.04 & 1.41 & 14.99 & 0.24\end{array}$

Deoxy $\quad \beta$-chain $\quad 34^{\circ} \mathrm{C} \quad 500 \mathrm{MHz}$ 


\begin{tabular}{|c|c|c|c|c|}
\hline 4 & 52.64 & 1.56 & 18.89 & 0.56 \\
\hline 6 & 50.76 & 1.10 & 18.03 & 0.39 \\
\hline 7 & 54.17 & 1.60 & 18.98 & 0.56 \\
\hline 8 & 51.90 & 1.50 & 18.93 & 0.55 \\
\hline 9 & 51.23 & 1.08 & 18.69 & 0.39 \\
\hline 10 & 54.74 & 1.30 & 19.38 & 0.46 \\
\hline 11 & 55.00 & 1.23 & 18.63 & 0.42 \\
\hline 13 & 52.63 & 1.26 & 19.58 & 0.47 \\
\hline 15 & 54.61 & 2.49 & 19.66 & 0.90 \\
\hline 16 & 52.55 & 1.38 & 19.11 & 0.50 \\
\hline 17 & 56.17 & 1.56 & 18.77 & 0.52 \\
\hline 18 & 53.27 & 1.01 & 18.04 & 0.34 \\
\hline 23 & 51.41 & 1.38 & 18.88 & 0.51 \\
\hline 25 & 50.21 & 1.18 & 18.99 & 0.45 \\
\hline 26 & 52.94 & 1.37 & 19.44 & 0.50 \\
\hline 27 & 54.38 & 1.62 & 19.45 & 0.58 \\
\hline 29 & 54.99 & 1.66 & 19.34 & 0.58 \\
\hline 30 & 55.01 & 2.73 & 18.38 & 0.91 \\
\hline 32 & 53.12 & 5.36 & 19.90 & 2.01 \\
\hline 35 & 55.50 & 2.76 & 18.86 & 0.94 \\
\hline 37 & 55.22 & 1.76 & 20.01 & 0.64 \\
\hline 40 & 55.48 & 2.15 & 18.99 & 0.73 \\
\hline 42 & 53.71 & 2.86 & 17.57 & 0.94 \\
\hline 45 & 53.15 & 1.55 & 18.94 & 0.55 \\
\hline 53 & 52.76 & 1.45 & 18.49 & 0.51 \\
\hline 54 & 52.48 & 1.12 & 18.46 & 0.40 \\
\hline 55 & 50.15 & 1.20 & 18.17 & 0.44 \\
\hline 56 & 49.16 & 1.04 & 17.88 & 0.38 \\
\hline 59 & 52.99 & 0.98 & 19.98 & 0.37 \\
\hline 60 & 54.28 & 1.85 & 19.74 & 0.67 \\
\hline
\end{tabular}




\begin{tabular}{|c|c|c|c|c|}
\hline 61 & 53.71 & 1.04 & 19.02 & 0.37 \\
\hline 62 & 52.94 & 1.12 & 19.83 & 0.42 \\
\hline 65 & 56.02 & 2.48 & 18.39 & 0.81 \\
\hline 72 & 51.02 & 2.23 & 20.38 & 0.89 \\
\hline 74 & 49.32 & 1.52 & 18.78 & 0.58 \\
\hline 76 & 49.80 & 1.02 & 19.27 & 0.40 \\
\hline 78 & 54.56 & 1.77 & 18.99 & 0.62 \\
\hline 80 & 49.15 & 1.13 & 18.53 & 0.43 \\
\hline 82 & 52.72 & 2.31 & 19.04 & 0.84 \\
\hline 83 & 50.10 & 1.86 & 18.28 & 0.68 \\
\hline 85 & 52.57 & 1.95 & 19.43 & 0.72 \\
\hline 86 & 50.88 & 1.89 & 18.44 & 0.68 \\
\hline 94 & 50.76 & 3.06 & 18.95 & 1.14 \\
\hline 101 & 51.61 & 1.71 & 20.22 & 0.67 \\
\hline 105 & 55.76 & 2.83 & 19.81 & 1.01 \\
\hline 112 & 56.71 & 1.53 & 20.41 & 0.55 \\
\hline 113 & 55.08 & 6.70 & 20.03 & 2.44 \\
\hline 116 & 56.24 & 1.08 & 19.71 & 0.38 \\
\hline 118 & 56.37 & 1.76 & 18.19 & 0.57 \\
\hline 121 & 50.54 & 0.91 & 19.30 & 0.35 \\
\hline 126 & 55.26 & 1.19 & 19.04 & 0.41 \\
\hline 127 & 55.82 & 1.35 & 20.36 & 0.49 \\
\hline 128 & 55.03 & 1.33 & 19.48 & 0.47 \\
\hline 133 & 55.70 & 2.14 & 19.59 & 0.75 \\
\hline 135 & 55.78 & 2.15 & 19.48 & 0.75 \\
\hline 136 & 53.97 & 1.48 & 18.16 & 0.50 \\
\hline 137 & 55.90 & 1.47 & 20.12 & 0.53 \\
\hline 139 & 53.81 & 1.65 & 17.85 & 0.55 \\
\hline 140 & 55.02 & 1.97 & 19.02 & 0.68 \\
\hline 144 & 53.06 & 0.86 & 17.67 & 0.29 \\
\hline
\end{tabular}




\begin{tabular}{|c|c|c|c|c|}
\hline Deoxy & $\beta$-chain & $34^{\circ} \mathrm{C}$ & $600 \mathrm{MHz}$ & \\
\hline 4 & 72.58 & 2.30 & 16.38 & 0.52 \\
\hline 6 & 73.46 & 1.36 & 15.01 & 0.28 \\
\hline 7 & 72.05 & 1.79 & 15.51 & 0.38 \\
\hline 8 & 73.29 & 2.04 & 16.19 & 0.45 \\
\hline 9 & 71.40 & 1.30 & 15.44 & 0.28 \\
\hline 10 & 73.09 & 1.36 & 15.80 & 0.30 \\
\hline 11 & 74.67 & 1.42 & 15.05 & 0.29 \\
\hline 13 & 73.90 & 1.60 & 15.84 & 0.34 \\
\hline 15 & 76.92 & 2.88 & 16.49 & 0.62 \\
\hline 16 & 74.46 & 1.63 & 15.48 & 0.34 \\
\hline 17 & 78.06 & 1.81 & 15.53 & 0.36 \\
\hline 18 & 71.17 & 1.09 & 14.60 & 0.22 \\
\hline 23 & 72.23 & 1.77 & 16.09 & 0.39 \\
\hline 25 & 68.89 & 1.47 & 15.81 & 0.34 \\
\hline 26 & 72.31 & 1.59 & 15.23 & 0.33 \\
\hline 27 & 74.22 & 2.18 & 15.50 & 0.46 \\
\hline 29 & 79.26 & 2.37 & 15.48 & 0.46 \\
\hline 30 & 74.54 & 1.92 & 15.91 & 0.41 \\
\hline 31 & 77.13 & 5.67 & 15.69 & 1.15 \\
\hline 32 & 80.09 & 8.18 & 15.02 & 1.53 \\
\hline 33 & 72.59 & 3.98 & 15.16 & 0.83 \\
\hline 35 & 79.66 & 3.40 & 14.52 & 0.62 \\
\hline 37 & 73.53 & 2.11 & 17.08 & 0.49 \\
\hline 38 & 69.50 & 2.76 & 17.10 & 0.68 \\
\hline 39 & 73.41 & 1.81 & 16.84 & 0.42 \\
\hline 40 & 72.22 & 2.74 & 16.09 & 0.61 \\
\hline 53 & 70.03 & 1.54 & 15.27 & 0.34 \\
\hline
\end{tabular}




\begin{tabular}{|c|c|c|c|c|}
\hline 54 & 71.73 & 1.35 & 14.92 & 0.28 \\
\hline 55 & 68.00 & 1.42 & 15.47 & 0.32 \\
\hline 59 & 71.30 & 1.27 & 16.91 & 0.30 \\
\hline 60 & 71.62 & 2.04 & 16.85 & 0.48 \\
\hline 61 & 75.23 & 1.39 & 15.92 & 0.29 \\
\hline 62 & 71.68 & 1.53 & 16.38 & 0.35 \\
\hline 65 & 69.31 & 3.66 & 16.17 & 0.85 \\
\hline 72 & 68.45 & 3.65 & 17.04 & 0.91 \\
\hline 74 & 68.03 & 1.90 & 16.07 & 0.45 \\
\hline 76 & 66.87 & 1.19 & 16.52 & 0.29 \\
\hline 78 & 72.68 & 2.06 & 16.12 & 0.46 \\
\hline 82 & 71.65 & 2.67 & 16.44 & 0.61 \\
\hline 83 & 69.33 & 2.44 & 15.58 & 0.55 \\
\hline 85 & 68.26 & 2.33 & 16.32 & 0.56 \\
\hline 86 & 71.81 & 2.65 & 15.59 & 0.58 \\
\hline 87 & 71.66 & 4.09 & 14.71 & 0.84 \\
\hline 94 & 77.48 & 5.05 & 14.66 & 0.96 \\
\hline 101 & 73.69 & 2.40 & 16.42 & 0.53 \\
\hline 105 & 79.18 & 4.09 & 16.25 & 0.84 \\
\hline 106 & 78.87 & 8.25 & 15.27 & 1.60 \\
\hline 118 & 78.35 & 2.02 & 15.38 & 0.40 \\
\hline 121 & 68.05 & 0.97 & 16.20 & 0.23 \\
\hline 122 & 67.32 & 1.18 & 14.87 & 0.26 \\
\hline 126 & 75.95 & 1.23 & 15.31 & 0.25 \\
\hline 128 & 78.33 & 1.72 & 16.43 & 0.36 \\
\hline 135 & 72.85 & 2.83 & 16.85 & 0.66 \\
\hline 136 & 75.94 & 1.96 & 14.97 & 0.39 \\
\hline 137 & 75.60 & 1.88 & 16.84 & 0.42 \\
\hline 140 & 78.94 & 2.72 & 15.42 & 0.53 \\
\hline 144 & 69.98 & 1.00 & 14.61 & 0.21 \\
\hline
\end{tabular}




\begin{tabular}{|c|c|c|c|c|}
\hline $\mathrm{CO}$ & $\alpha$-chain & $29^{\circ} \mathrm{C}$ & $500 \mathrm{MHz}$ & \\
\hline 6 & 72.00 & 2.98 & 19.32 & 0.80 \\
\hline 8 & 68.12 & 3.47 & 18.92 & 0.96 \\
\hline 10 & 72.59 & 2.70 & 19.25 & 0.72 \\
\hline 11 & 71.17 & 3.66 & 19.25 & 0.99 \\
\hline 15 & 68.77 & 3.06 & 19.76 & 0.88 \\
\hline 16 & 71.88 & 1.74 & 18.62 & 0.45 \\
\hline 17 & 65.13 & 1.93 & 19.20 & 0.57 \\
\hline 21 & 64.68 & 1.98 & 20.07 & 0.62 \\
\hline 24 & 68.40 & 3.07 & 19.58 & 0.88 \\
\hline 25 & 67.25 & 2.43 & 18.89 & 0.68 \\
\hline 26 & 70.87 & 3.61 & 20.36 & 1.04 \\
\hline 31 & 65.44 & 3.05 & 18.59 & 0.87 \\
\hline 32 & 72.30 & 2.99 & 19.48 & 0.81 \\
\hline 33 & 67.63 & 3.63 & 19.87 & 1.07 \\
\hline 36 & 70.74 & 2.64 & 18.69 & 0.70 \\
\hline 38 & 68.22 & 6.78 & 20.12 & 2.00 \\
\hline 42 & 66.45 & 4.20 & 19.74 & 1.25 \\
\hline 47 & 65.90 & 1.35 & 18.44 & 0.38 \\
\hline 55 & 68.56 & 2.72 & 19.33 & 0.77 \\
\hline 57 & 64.28 & 2.08 & 19.16 & 0.62 \\
\hline 58 & 66.51 & 2.39 & 20.41 & 0.73 \\
\hline 59 & 67.95 & 2.88 & 20.18 & 0.86 \\
\hline 61 & 67.46 & 2.56 & 18.59 & 0.70 \\
\hline 63 & 72.27 & 1.61 & 19.39 & 0.43 \\
\hline 65 & 67.00 & 2.59 & 20.27 & 0.78 \\
\hline 66 & 71.16 & 2.48 & 19.32 & 0.67 \\
\hline 67 & 66.39 & 3.43 & 18.37 & 0.95 \\
\hline
\end{tabular}




$\begin{array}{lllll}69 & 67.39 & 2.50 & 19.22 & 0.71 \\ 70 & 67.92 & 2.95 & 18.30 & 0.79 \\ 79 & 70.49 & 1.65 & 18.84 & 0.44 \\ 84 & 70.63 & 3.11 & 19.92 & 0.88 \\ 87 & 66.07 & 7.53 & 19.12 & 2.18 \\ 90 & 68.17 & 4.34 & 19.22 & 1.22 \\ 92 & 64.61 & 3.83 & 18.98 & 1.13 \\ 100 & 72.83 & 3.26 & 19.47 & 0.87 \\ 103 & 72.22 & 2.34 & 19.98 & 0.65 \\ 107 & 66.10 & 1.88 & 19.20 & 0.55 \\ 109 & 70.51 & 2.45 & 19.44 & 0.67 \\ 117 & 71.97 & 3.15 & 18.06 & 0.79 \\ 122 & 71.29 & 2.66 & 19.80 & 0.74 \\ 124 & 67.77 & 2.68 & 18.61 & 0.74 \\ 125 & 72.69 & 2.51 & 20.27 & 0.70 \\ 126 & 72.45 & 3.15 & 20.05 & 0.87 \\ 129 & 72.91 & 4.74 & 20.25 & 1.32 \\ 130 & 70.66 & 3.87 & 19.70 & 1.08 \\ 131 & 72.62 & 3.03 & 19.04 & 0.79\end{array}$

CO $\alpha$-chain $\quad 29^{\circ} \mathrm{C} \quad 600 \mathrm{MHz}$

$\begin{array}{lllll}6 & 95.99 & 5.35 & 16.37 & 0.91 \\ 7 & 95.07 & 7.31 & 17.33 & 1.33 \\ 8 & 95.28 & 5.28 & 15.78 & 0.87 \\ 9 & 96.04 & 6.84 & 17.02 & 1.21 \\ 10 & 100.76 & 4.13 & 17.10 & 0.70 \\ 11 & 96.30 & 5.62 & 17.48 & 1.02 \\ 13 & 96.49 & 3.36 & 16.46 & 0.57 \\ 14 & 92.19 & 4.97 & 16.03 & 0.86\end{array}$




\begin{tabular}{|c|c|c|c|c|}
\hline 15 & 91.11 & 4.74 & 17.64 & 0.92 \\
\hline 16 & 97.78 & 2.85 & 16.20 & 0.47 \\
\hline 21 & 88.08 & 3.02 & 17.29 & 0.59 \\
\hline 22 & 88.20 & 5.05 & 15.56 & 0.89 \\
\hline 23 & 86.71 & 2.42 & 15.81 & 0.44 \\
\hline 24 & 94.57 & 5.12 & 16.52 & 0.90 \\
\hline 25 & 89.13 & 3.58 & 15.95 & 0.64 \\
\hline 26 & 99.91 & 4.42 & 17.37 & 0.77 \\
\hline 28 & 95.25 & 3.80 & 17.37 & 0.69 \\
\hline 30 & 96.96 & 4.75 & 16.94 & 0.83 \\
\hline 32 & 97.62 & 4.35 & 16.97 & 0.76 \\
\hline 33 & 95.27 & 6.06 & 16.33 & 1.04 \\
\hline 39 & 98.48 & 12.51 & 17.21 & 2.19 \\
\hline 42 & 88.44 & 6.25 & 17.20 & 1.22 \\
\hline 46 & 99.57 & 3.49 & 15.85 & 0.56 \\
\hline 55 & 90.54 & 3.92 & 16.90 & 0.73 \\
\hline 56 & 93.12 & 2.31 & 16.98 & 0.42 \\
\hline 57 & 86.15 & 2.67 & 16.83 & 0.52 \\
\hline 59 & 96.43 & 4.15 & 16.37 & 0.70 \\
\hline 61 & 88.89 & 3.71 & 15.83 & 0.66 \\
\hline 63 & 96.59 & 2.33 & 16.80 & 0.40 \\
\hline 65 & 89.45 & 3.52 & 17.56 & 0.69 \\
\hline 66 & 94.11 & 4.26 & 16.36 & 0.74 \\
\hline 68 & 96.48 & 2.47 & 17.02 & 0.44 \\
\hline 69 & 88.64 & 3.79 & 16.70 & 0.71 \\
\hline 73 & 92.01 & 3.98 & 16.70 & 0.72 \\
\hline 74 & 89.07 & 2.74 & 16.93 & 0.52 \\
\hline 84 & 94.70 & 4.81 & 17.11 & 0.87 \\
\hline 90 & 90.20 & 7.91 & 17.38 & 1.52 \\
\hline 100 & 97.81 & 6.35 & 16.20 & 1.05 \\
\hline
\end{tabular}




$\begin{array}{lllll}103 & 100.74 & 4.58 & 17.43 & 0.79 \\ 110 & 94.67 & 2.65 & 16.62 & 0.46 \\ 115 & 87.18 & 5.01 & 15.97 & 0.92 \\ 117 & 100.21 & 5.41 & 16.27 & 0.88 \\ 122 & 97.29 & 3.95 & 17.16 & 0.70 \\ 125 & 99.45 & 4.00 & 16.96 & 0.68 \\ 126 & 93.97 & 5.04 & 16.74 & 0.90 \\ 127 & 99.88 & 8.73 & 16.54 & 1.45 \\ 128 & 100.59 & 4.15 & 16.91 & 0.70 \\ 129 & 99.66 & 7.44 & 17.25 & 1.29 \\ 130 & 97.90 & 7.19 & 17.27 & 1.27 \\ 131 & 98.50 & 4.74 & 16.40 & 0.79 \\ 134 & 96.08 & 6.30 & 17.35 & 1.14\end{array}$

CO $\alpha$-chain $\quad 34^{\circ} \mathrm{C} \quad 500 \mathrm{MHz}$

$\begin{array}{lllll}10 & 53.13 & 1.57 & 19.19 & 0.57 \\ 11 & 52.60 & 2.55 & 20.29 & 0.98 \\ 12 & 54.12 & 1.79 & 19.74 & 0.65 \\ 14 & 54.58 & 1.92 & 19.91 & 0.70 \\ 16 & 53.36 & 1.10 & 18.64 & 0.39 \\ 17 & 49.72 & 0.96 & 18.44 & 0.36 \\ 21 & 50.86 & 1.14 & 19.61 & 0.44 \\ 22 & 53.91 & 4.39 & 19.02 & 1.55 \\ 23 & 49.33 & 1.08 & 18.90 & 0.41 \\ 24 & 49.84 & 1.66 & 19.72 & 0.65 \\ 25 & 50.85 & 1.46 & 19.23 & 0.55 \\ 26 & 53.77 & 1.76 & 20.21 & 0.66 \\ 28 & 51.35 & 1.67 & 19.93 & 0.65 \\ 29 & 50.50 & 2.41 & 18.18 & 0.87\end{array}$




\begin{tabular}{|c|c|c|c|c|}
\hline 31 & 48.71 & 1.88 & 18.91 & 0.73 \\
\hline 33 & 53.92 & 2.22 & 19.67 & 0.81 \\
\hline 36 & 52.21 & 1.54 & 18.54 & 0.55 \\
\hline 42 & 50.67 & 2.72 & 19.17 & 1.03 \\
\hline 46 & 54.70 & 1.31 & 18.85 & 0.45 \\
\hline 55 & 51.15 & 1.61 & 19.28 & 0.61 \\
\hline 57 & 49.12 & 1.25 & 19.99 & 0.51 \\
\hline 58 & 52.78 & 1.57 & 19.90 & 0.59 \\
\hline 59 & 53.19 & 1.67 & 19.73 & 0.62 \\
\hline 60 & 51.24 & 1.43 & 20.08 & 0.56 \\
\hline 61 & 50.48 & 1.58 & 18.54 & 0.58 \\
\hline 63 & 53.59 & 0.91 & 20.07 & 0.34 \\
\hline 65 & 53.35 & 1.69 & 19.14 & 0.61 \\
\hline 66 & 53.09 & 1.54 & 18.73 & 0.54 \\
\hline 67 & 52.35 & 1.96 & 18.85 & 0.71 \\
\hline 69 & 50.89 & 1.64 & 19.94 & 0.64 \\
\hline 70 & 49.42 & 1.81 & 18.57 & 0.68 \\
\hline 71 & 50.36 & 0.82 & 19.48 & 0.32 \\
\hline 73 & 51.27 & 1.37 & 18.03 & 0.48 \\
\hline 74 & 50.51 & 1.17 & 18.55 & 0.43 \\
\hline 79 & 52.32 & 1.20 & 18.77 & 0.43 \\
\hline 87 & 52.01 & 4.94 & 19.29 & 1.83 \\
\hline 100 & 55.35 & 2.27 & 18.94 & 0.78 \\
\hline 103 & 54.20 & 1.90 & 19.45 & 0.68 \\
\hline 109 & 53.75 & 1.63 & 19.48 & 0.59 \\
\hline 110 & 52.99 & 1.24 & 19.01 & 0.45 \\
\hline 115 & 52.24 & 1.98 & 18.12 & 0.69 \\
\hline 117 & 55.40 & 2.01 & 18.96 & 0.69 \\
\hline 120 & 53.78 & 1.01 & 18.72 & 0.35 \\
\hline 122 & 54.81 & 1.72 & 19.86 & 0.62 \\
\hline
\end{tabular}




$\begin{array}{lllll}133 & 52.99 & 3.46 & 18.07 & 1.18 \\ 134 & 55.35 & 2.50 & 19.60 & 0.88\end{array}$

CO $\alpha$-chain $\quad 34^{\circ} \mathrm{C} \quad 600 \mathrm{MHz}$

\begin{tabular}{lllll}
6 & 78.14 & 3.59 & 16.39 & 0.75 \\
7 & 76.56 & 3.64 & 16.63 & 0.79 \\
8 & 71.97 & 3.00 & 15.30 & 0.64 \\
9 & 79.36 & 4.09 & 16.07 & 0.83 \\
10 & 78.56 & 2.45 & 17.13 & 0.53 \\
14 & 74.44 & 3.05 & 15.96 & 0.65 \\
15 & 72.31 & 2.68 & 17.15 & 0.64 \\
16 & 78.61 & 1.89 & 16.20 & 0.39 \\
17 & 71.75 & 1.40 & 15.92 & 0.31 \\
21 & 71.48 & 1.80 & 16.47 & 0.41 \\
25 & 76.15 & 2.28 & 16.68 & 0.50 \\
28 & 76.54 & 2.28 & 17.20 & 0.51 \\
30 & 77.49 & 3.17 & 17.05 & 0.70 \\
32 & 75.46 & 2.52 & 17.46 & 0.58 \\
33 & 73.04 & 2.74 & 16.41 & 0.62 \\
36 & 72.56 & 1.97 & 15.42 & 0.42 \\
39 & 72.05 & 6.81 & 16.39 & 1.55 \\
46 & 73.51 & 1.93 & 15.76 & 0.41 \\
47 & 71.21 & 1.13 & 15.60 & 0.25 \\
54 & 75.20 & 2.45 & 16.82 & 0.55 \\
55 & 75.25 & 2.65 & 17.12 & 0.60 \\
56 & 70.25 & 2.97 & 17.42 & 0.74 \\
57 & 69.00 & 1.74 & 17.25 & 0.44 \\
\hline 9 & 76.07 & 2.37 & 16.73 & 0.52 \\
\hline 5 & 70.08 & 2.25 & 16.28 & 0.52
\end{tabular}




$\begin{array}{lllll}63 & 79.55 & 1.54 & 16.98 & 0.33 \\ 65 & 70.62 & 2.27 & 16.00 & 0.51 \\ 66 & 75.44 & 2.26 & 16.31 & 0.49 \\ 67 & 74.59 & 2.96 & 15.58 & 0.62 \\ 69 & 73.03 & 2.51 & 16.06 & 0.55 \\ 79 & 74.91 & 1.99 & 16.69 & 0.44 \\ 90 & 70.24 & 4.56 & 16.12 & 1.05 \\ 98 & 78.73 & 3.97 & 16.80 & 0.85 \\ 100 & 78.13 & 4.25 & 16.25 & 0.88 \\ 107 & 72.76 & 1.96 & 16.07 & 0.43 \\ 108 & 74.56 & 4.54 & 15.37 & 0.94 \\ 110 & 79.69 & 2.39 & 16.94 & 0.51 \\ 115 & 73.31 & 3.83 & 15.44 & 0.81 \\ 117 & 76.55 & 2.70 & 15.92 & 0.56 \\ 120 & 73.59 & 1.68 & 15.44 & 0.35 \\ 121 & 77.34 & 2.56 & 16.72 & 0.55 \\ 122 & 77.79 & 2.55 & 17.11 & 0.56 \\ 125 & 78.37 & 2.31 & 17.39 & 0.51 \\ 126 & 78.05 & 3.14 & 16.30 & 0.66 \\ 127 & 75.13 & 5.06 & 15.83 & 1.07 \\ 130 & 75.96 & 4.63 & 16.50 & 1.01 \\ 131 & 77.35 & 3.06 & 15.87 & 0.63 \\ 132 & 78.57 & 3.18 & 17.36 & 0.70\end{array}$

CO $\beta$-chain $29^{\circ} \mathrm{C} \quad 500 \mathrm{MHz}$

$\begin{array}{ccccc}7 & 68.00 & 2.70 & 18.96 & 0.75 \\ 8 & 67.21 & 1.86 & 18.74 & 0.52 \\ 10 & 68.33 & 1.63 & 19.49 & 0.47 \\ 12 & 68.38 & 1.84 & 18.56 & 0.50\end{array}$




\begin{tabular}{|c|c|c|c|c|}
\hline 16 & 66.14 & 2.02 & 18.65 & 0.57 \\
\hline 18 & 69.26 & 1.84 & 18.51 & 0.49 \\
\hline 25 & 65.27 & 2.26 & 18.26 & 0.63 \\
\hline 26 & 70.95 & 2.14 & 20.01 & 0.60 \\
\hline 32 & 70.21 & 6.02 & 18.19 & 1.56 \\
\hline 33 & 70.12 & 5.61 & 17.67 & 1.41 \\
\hline 34 & 64.39 & 5.53 & 19.13 & 1.64 \\
\hline 35 & 67.82 & 4.76 & 18.41 & 1.29 \\
\hline 38 & 69.28 & 5.14 & 20.28 & 1.51 \\
\hline 45 & 66.72 & 2.02 & 18.60 & 0.56 \\
\hline 53 & 66.63 & 2.31 & 19.29 & 0.67 \\
\hline 54 & 66.72 & 1.35 & 18.25 & 0.37 \\
\hline 55 & 64.47 & 1.96 & 18.45 & 0.56 \\
\hline 56 & 65.45 & 1.76 & 17.97 & 0.48 \\
\hline 57 & 71.02 & 1.34 & 17.75 & 0.34 \\
\hline 62 & 68.11 & 1.15 & 19.42 & 0.33 \\
\hline 63 & 71.03 & 2.67 & 19.13 & 0.72 \\
\hline 66 & 67.66 & 2.37 & 18.86 & 0.66 \\
\hline 67 & 71.02 & 3.16 & 19.35 & 0.86 \\
\hline 68 & 68.81 & 2.30 & 19.77 & 0.66 \\
\hline 69 & 66.01 & 2.19 & 18.96 & 0.63 \\
\hline 71 & 68.16 & 2.67 & 18.29 & 0.72 \\
\hline 72 & 68.47 & 2.78 & 17.60 & 0.71 \\
\hline 73 & 69.31 & 2.34 & 19.62 & 0.66 \\
\hline 82 & 65.41 & 2.84 & 18.37 & 0.80 \\
\hline 86 & 69.09 & 1.97 & 18.97 & 0.54 \\
\hline 90 & 72.08 & 3.46 & 19.87 & 0.95 \\
\hline 92 & 72.11 & 3.51 & 18.46 & 0.90 \\
\hline 93 & 66.95 & 4.78 & 17.83 & 1.27 \\
\hline 102 & 72.35 & 4.19 & 18.97 & 1.10 \\
\hline
\end{tabular}




\begin{tabular}{|c|c|c|c|c|}
\hline 104 & 73.00 & 4.01 & 19.14 & 1.05 \\
\hline 117 & 74.06 & 2.35 & 19.19 & 0.61 \\
\hline 122 & 65.96 & 1.56 & 18.74 & 0.44 \\
\hline 126 & 74.29 & 1.92 & 19.10 & 0.49 \\
\hline 128 & 74.54 & 3.24 & 20.23 & 0.88 \\
\hline 133 & 72.52 & 4.85 & 18.64 & 1.25 \\
\hline 137 & 75.09 & 3.60 & 19.76 & 0.95 \\
\hline 138 & 72.43 & 3.25 & 20.27 & 0.91 \\
\hline 139 & 72.25 & 2.75 & 18.50 & 0.70 \\
\hline 140 & 72.73 & 3.17 & 20.28 & 0.88 \\
\hline $\mathrm{CO}$ & $\beta$-chain & $29^{\circ} \mathrm{C}$ & $600 \mathrm{MHz}$ & \\
\hline 4 & 79.37 & 1.53 & 14.88 & 0.29 \\
\hline 7 & 92.05 & 2.89 & 16.24 & 0.51 \\
\hline 8 & 85.74 & 2.30 & 15.78 & 0.42 \\
\hline 9 & 82.02 & 1.97 & 15.74 & 0.38 \\
\hline 10 & 92.43 & 1.98 & 16.62 & 0.36 \\
\hline 16 & 87.78 & 2.64 & 16.08 & 0.48 \\
\hline 18 & 90.86 & 2.50 & 15.72 & 0.43 \\
\hline 26 & 96.15 & 2.82 & 17.61 & 0.52 \\
\hline 28 & 91.59 & 2.86 & 15.93 & 0.50 \\
\hline 29 & 97.13 & 3.52 & 16.49 & 0.60 \\
\hline 34 & 87.48 & 8.79 & 17.09 & 1.72 \\
\hline 35 & 92.60 & 7.42 & 14.96 & 1.20 \\
\hline 38 & 96.70 & 7.78 & 16.90 & 1.36 \\
\hline 53 & 88.92 & 2.74 & 17.37 & 0.54 \\
\hline 57 & 94.10 & 2.02 & 15.74 & 0.34 \\
\hline 60 & 92.55 & 4.18 & 14.96 & 0.68 \\
\hline 62 & 86.68 & 2.08 & 16.78 & 0.40 \\
\hline
\end{tabular}




\begin{tabular}{|c|c|c|c|c|}
\hline 65 & 88.57 & 2.65 & 17.86 & 0.53 \\
\hline 66 & 89.42 & 2.96 & 15.70 & 0.52 \\
\hline 67 & 92.38 & 4.00 & 16.06 & 0.70 \\
\hline 69 & 87.39 & 2.93 & 16.77 & 0.56 \\
\hline 70 & 90.93 & 3.03 & 17.28 & 0.58 \\
\hline 71 & 87.53 & 3.43 & 15.51 & 0.61 \\
\hline 72 & 88.50 & 3.44 & 15.17 & 0.59 \\
\hline 73 & 95.50 & 3.22 & 16.61 & 0.56 \\
\hline 74 & 90.10 & 3.01 & 16.74 & 0.56 \\
\hline 82 & 80.59 & 3.86 & 17.28 & 0.83 \\
\hline 85 & 79.58 & 2.68 & 15.34 & 0.52 \\
\hline 86 & 90.88 & 2.40 & 16.65 & 0.44 \\
\hline 88 & 87.91 & 3.61 & 18.04 & 0.74 \\
\hline 89 & 93.13 & 4.24 & 17.78 & 0.81 \\
\hline 90 & 95.21 & 3.85 & 16.79 & 0.68 \\
\hline 92 & 88.91 & 4.84 & 16.75 & 0.91 \\
\hline 93 & 81.97 & 6.10 & 15.87 & 1.18 \\
\hline 97 & 81.28 & 3.60 & 16.17 & 0.72 \\
\hline 99 & 84.55 & 4.45 & 17.89 & 0.94 \\
\hline 102 & 94.75 & 5.76 & 16.79 & 1.02 \\
\hline 106 & 99.23 & 5.14 & 16.68 & 0.86 \\
\hline 116 & 97.67 & 2.77 & 15.70 & 0.45 \\
\hline 117 & 95.51 & 3.06 & 15.74 & 0.50 \\
\hline 118 & 95.65 & 3.18 & 15.08 & 0.50 \\
\hline 122 & 86.45 & 1.86 & 16.28 & 0.35 \\
\hline 127 & 88.98 & 3.31 & 16.61 & 0.62 \\
\hline 128 & 98.97 & 3.68 & 17.46 & 0.65 \\
\hline 130 & 98.52 & 4.97 & 15.61 & 0.79 \\
\hline 135 & 99.16 & 4.59 & 17.91 & 0.83 \\
\hline 136 & 98.11 & 5.39 & 16.49 & 0.91 \\
\hline
\end{tabular}




$\begin{array}{lllll}137 & 83.86 & 1.94 & 15.22 & 0.35 \\ 138 & 95.76 & 4.50 & 17.38 & 0.82 \\ 139 & 91.27 & 4.12 & 15.57 & 0.70\end{array}$

CO $\quad \beta$-chain $\quad 34^{\circ} \mathrm{C} \quad 500 \mathrm{MHz}$

$\begin{array}{lllll}7 & 53.08 & 1.82 & 19.43 & 0.67 \\ 8 & 51.52 & 1.41 & 19.61 & 0.54 \\ 9 & 52.58 & 1.34 & 18.99 & 0.48 \\ 10 & 52.81 & 1.30 & 19.58 & 0.48 \\ 12 & 52.39 & 1.23 & 18.61 & 0.44 \\ 13 & 50.99 & 1.55 & 19.41 & 0.59 \\ 16 & 52.70 & 1.63 & 19.10 & 0.59 \\ 18 & 53.93 & 1.31 & 18.46 & 0.45 \\ 26 & 55.88 & 1.61 & 20.52 & 0.59 \\ 27 & 53.86 & 2.18 & 19.85 & 0.80 \\ 28 & 55.56 & 2.14 & 19.67 & 0.76 \\ 29 & 58.41 & 2.08 & 20.33 & 0.73 \\ 30 & 57.35 & 1.55 & 19.76 & 0.53 \\ 31 & 55.12 & 2.69 & 20.31 & 0.99 \\ 32 & 55.81 & 5.25 & 18.32 & 1.72 \\ 53 & 51.94 & 1.84 & 19.52 & 0.69 \\ 54 & 51.60 & 1.20 & 18.70 & 0.44 \\ 55 & 51.13 & 1.46 & 18.47 & 0.53 \\ 62 & 53.33 & 1.34 & 19.39 & 0.49 \\ 63 & 52.76 & 2.07 & 19.89 & 0.78 \\ 64 & 53.25 & 1.69 & 20.21 & 0.64 \\ 66 & 51.34 & 1.71 & 19.86 & 0.66 \\ 67 & 52.19 & 2.06 & 19.74 & 0.78 \\ 68 & 55.31 & 1.89 & 20.31 & 0.69\end{array}$




$\begin{array}{lllll}70 & 52.36 & 1.96 & 19.55 & 0.73 \\ 71 & 52.39 & 2.10 & 18.74 & 0.75 \\ 73 & 51.83 & 1.68 & 19.99 & 0.65 \\ 78 & 52.89 & 1.44 & 19.94 & 0.54 \\ 86 & 52.97 & 1.51 & 18.75 & 0.53 \\ 88 & 52.59 & 2.27 & 20.54 & 0.89 \\ 89 & 54.52 & 2.28 & 21.16 & 0.89 \\ 90 & 57.90 & 2.29 & 19.54 & 0.77 \\ 93 & 53.23 & 3.90 & 19.35 & 1.42 \\ 113 & 57.81 & 5.57 & 19.58 & 1.89 \\ 117 & 58.88 & 1.75 & 19.54 & 0.58 \\ 122 & 51.27 & 1.16 & 18.95 & 0.43 \\ 126 & 54.89 & 1.46 & 18.98 & 0.50 \\ 127 & 55.81 & 1.89 & 20.90 & 0.71 \\ 130 & 57.89 & 2.65 & 19.61 & 0.90 \\ 135 & 53.11 & 2.47 & 20.74 & 0.97 \\ 137 & 58.53 & 2.75 & 19.49 & 0.92 \\ 138 & 58.75 & 2.69 & 20.94 & 0.96 \\ 139 & 55.41 & 2.17 & 19.03 & 0.75\end{array}$

Co $\quad \beta$-chain $\quad 34^{\circ} \mathrm{C} \quad 600 \mathrm{MHz}$

$\begin{array}{lllll}6 & 74.77 & 3.28 & 15.89 & 0.70 \\ 7 & 74.68 & 1.83 & 16.64 & 0.41 \\ 8 & 70.71 & 1.48 & 16.02 & 0.33 \\ 9 & 68.86 & 1.24 & 15.67 & 0.28 \\ 10 & 75.00 & 1.32 & 17.42 & 0.31 \\ 12 & 70.81 & 1.31 & 16.18 & 0.30 \\ 13 & 71.15 & 1.73 & 16.67 & 0.40 \\ 16 & 70.65 & 1.78 & 15.67 & 0.40\end{array}$




\begin{tabular}{|c|c|c|c|c|}
\hline 18 & 74.09 & 1.41 & 15.47 & 0.29 \\
\hline 25 & 68.86 & 1.67 & 16.74 & 0.41 \\
\hline 28 & 74.74 & 1.89 & 16.37 & 0.41 \\
\hline 29 & 77.57 & 2.21 & 16.63 & 0.47 \\
\hline 30 & 73.17 & 1.81 & 15.75 & 0.39 \\
\hline 31 & 75.44 & 1.63 & 16.88 & 0.36 \\
\hline 32 & 77.60 & 4.97 & 15.30 & 0.98 \\
\hline 33 & 76.81 & 4.15 & 15.55 & 0.84 \\
\hline 53 & 73.42 & 1.77 & 17.13 & 0.41 \\
\hline 54 & 71.22 & 1.29 & 15.20 & 0.28 \\
\hline 55 & 69.15 & 1.51 & 16.07 & 0.35 \\
\hline 57 & 74.81 & 1.15 & 15.69 & 0.24 \\
\hline 60 & 71.48 & 2.36 & 15.32 & 0.51 \\
\hline 62 & 71.37 & 1.51 & 16.79 & 0.35 \\
\hline 63 & 72.90 & 2.08 & 17.43 & 0.50 \\
\hline 64 & 74.39 & 1.86 & 17.07 & 0.43 \\
\hline 65 & 71.96 & 1.60 & 17.56 & 0.39 \\
\hline 66 & 70.91 & 1.87 & 16.47 & 0.43 \\
\hline 67 & 73.10 & 2.45 & 16.22 & 0.54 \\
\hline 69 & 69.79 & 1.87 & 16.76 & 0.45 \\
\hline 70 & 73.79 & 1.95 & 17.14 & 0.45 \\
\hline 71 & 70.61 & 2.20 & 15.33 & 0.48 \\
\hline 72 & 72.01 & 2.05 & 15.77 & 0.45 \\
\hline 73 & 73.69 & 1.97 & 17.12 & 0.46 \\
\hline 75 & 73.00 & 1.21 & 17.03 & 0.28 \\
\hline 82 & 70.01 & 3.92 & 16.88 & 0.95 \\
\hline 86 & 76.69 & 1.67 & 16.94 & 0.37 \\
\hline 90 & 77.99 & 2.55 & 17.01 & 0.56 \\
\hline 92 & 73.36 & 3.82 & 15.86 & 0.83 \\
\hline 93 & 73.58 & 3.84 & 15.57 & 0.81 \\
\hline
\end{tabular}




$\begin{array}{ccccc}97 & 69.24 & 2.20 & 15.62 & 0.50 \\ 104 & 78.09 & 3.34 & 16.89 & 0.72 \\ 112 & 79.04 & 2.43 & 15.79 & 0.49 \\ 116 & 79.16 & 1.56 & 15.96 & 0.32 \\ 117 & 78.18 & 1.75 & 15.90 & 0.36 \\ 122 & 70.53 & 1.22 & 16.87 & 0.29 \\ 126 & 77.55 & 1.48 & 16.13 & 0.31 \\ 127 & 76.91 & 2.37 & 17.28 & 0.53 \\ 130 & 76.99 & 2.61 & 15.87 & 0.54 \\ 131 & 77.91 & 2.81 & 16.77 & 0.60 \\ 133 & 77.55 & 3.15 & 15.63 & 0.63 \\ 139 & 74.31 & 2.38 & 16.00 & 0.51 \\ 142 & 72.92 & 2.16 & 16.59 & 0.49\end{array}$

END OF THE LIST 